\title{
Exceptional zero formulae and a conjecture of Perrin-Riou
}

\author{
Rodolfo Venerucci
}

\begin{abstract}
Let $A / \mathbf{Q}$ be an elliptic curve with split multiplicative reduction at a prime $p$. We prove (an analogue of) a conjecture of Perrin-Riou, relating $p$-adic Beilinson-Kato elements to Heegner points in $A(\mathbf{Q})$, and a large part of the rank-one case of the Mazur-Tate-Teitelbaum exceptional zero conjecture for the cyclotomic $p$-adic $L$-function of $A$. More generally, let $f$ be the weight-two newform associated with $A$, let $f_{\infty}$ be the Hida family of $f$, and let $L_{p}\left(f_{\infty}, k, s\right)$ be the Mazur-Kitagawa two-variable $p$-adic $L$-function attached to $f_{\infty}$. We prove a $p$-adic Gross-Zagier formula, expressing the quadratic term of the Taylor expansion of $L_{p}\left(f_{\infty}, k, s\right)$ at $(k, s)=(2,1)$ as a non-zero rational multiple of the extended height-weight of a Heegner point in $A(\mathbf{Q})$.
\end{abstract}

\section{Introduction}

Let $A$ be an elliptic curve over $\mathbf{Q}$ of conductor $N p$, with $p>3$ a prime of split multiplicative reduction. Fix algebraic closures $\overline{\mathbf{Q}}$ and $\overline{\mathbf{Q}}_{p}$ of $\mathbf{Q}$ and $\mathbf{Q}_{p}$ respectively, and an embedding $i_{p}: \overline{\mathbf{Q}} \hookrightarrow \overline{\mathbf{Q}}_{p}$. Assume throughout this paper that the $p$-torsion subgroup $A_{p}$ of $A(\overline{\mathbf{Q}})$ is an irreducible $\mathbf{F}_{p}\left[G_{\mathbf{Q}}\right]$-module, where $G_{\mathbf{Q}}:=\operatorname{Gal}(\overline{\mathbf{Q}} / \mathbf{Q})$.

For every $n \in \mathbf{N}$, write $\mathbf{Q}_{n} / \mathbf{Q}$ for the cyclic sub-extension of $\mathbf{Q}\left(\mu_{p^{n+1}}\right) / \mathbf{Q}$ of degree $p^{n}$ and let $\mathbf{Q}_{\infty}=\bigcup_{n \in \mathbf{N}} \mathbf{Q}_{n}$ be the cyclotomic $\mathbf{Z}_{p}$-extension of $\mathbf{Q}$. Denote by $G_{\infty}:=\operatorname{Gal}\left(\mathbf{Q}_{\infty} / \mathbf{Q}\right)$ the Galois group of $\mathbf{Q}_{\infty}$ over $\mathbf{Q}$ and by $\Lambda_{\text {cyc }}:=\mathbf{Z}_{p} \llbracket G_{\infty} \rrbracket$ the cyclotomic Iwasawa algebra. Associated with $A / \mathbf{Q}$ (and $i_{p}$ ) there is a $p$-adic $L$-function

$$
L_{p}(A / \mathbf{Q}) \in \Lambda_{\text {cyc }},
$$

interpolating the critical values $L(A / \mathbf{Q}, \chi, 1)$ of the Hasse-Weil $L$-function of $A / \mathbf{Q}$ twisted by finite order characters $\chi: G_{\infty} \rightarrow \overline{\mathbf{Q}}_{p}^{*}$. Thanks to the results of Kato and Coleman-Perrin-Riou, it is known that $L_{p}(A / \mathbf{Q})$ arises from an Euler system for the $p$-adic Tate module of $A / \mathbf{Q}$. More precisely, denote by $\mathbf{Q}_{p, \infty}=\bigcup_{n \in \mathbf{N}} \mathbf{Q}_{p, n}$ the cyclotomic $\mathbf{Z}_{p}$-extension of $\mathbf{Q}_{p}$ (with notations similar to those introduced above), and by $T_{p}(A)$ the $p$-adic Tate module of $A$. For $K=\mathbf{Q}$ or $\mathbf{Q}_{p}$, let $H_{\mathrm{Iw}}^{1}\left(K_{\infty}, T_{p}(A)\right)$ be the inverse limit of the cohomology groups $H^{1}\left(K_{n}, T_{p}(A)\right)$. The work of Coleman-Perrin-Riou yields a big dual exponential

$$
\mathcal{L}_{A}: H_{\mathrm{Iw}}^{1}\left(\mathbf{Q}_{p, \infty}, T_{p}(A)\right) \longrightarrow \Lambda_{\text {cyc }} .
$$

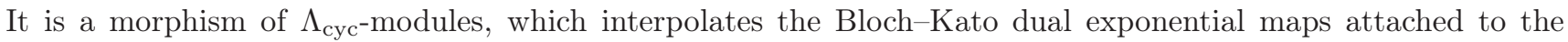
twists of $T_{p}(A)$ by finite order characters $\chi$ of $G_{\infty}$ (see Section 3.1 for the precise definition). In [Kat04] Kato constructs a cyclotomic Euler system for $T_{p}(A)$, related to $L_{p}(A / \mathbf{Q})$ via $\mathcal{L}_{A}$. In particular he constructs an element $\zeta_{\infty}^{\mathrm{BK}}=\left(\zeta_{n}^{\mathrm{BK}}\right)_{n \in \mathbf{N}} \in H_{\mathrm{Iw}}^{1}\left(\mathbf{Q}_{\infty}, T_{p}(A)\right)$ such that

$$
\mathcal{L}_{A}\left(\operatorname{res}_{p}\left(\zeta_{\infty}^{\mathrm{BK}}\right)\right)=L_{p}(A / \mathbf{Q})
$$

Kato's Euler system is built out of Steinberg symbols of certain Siegel modular units, which also appeared in the work of Beilinson. The classes $\zeta_{n}^{\mathrm{BK}}$ are then called p-adic Beilinson-Kato classes.

1.1. A conjecture of Perrin-Riou. Set $V_{p}(A):=T_{p}(A) \otimes \mathbf{z}_{p} \mathbf{Q}_{p}$ and denote by $\zeta^{\mathrm{BK}}$ the natural image of the class $\zeta_{0}^{\mathrm{BK}} \in H^{1}\left(\mathbf{Q}, T_{p}(A)\right)$ in $H^{1}\left(\mathbf{Q}, V_{p}(A)\right)$. We call $\zeta^{\mathrm{BK}}$ the $p$-adic Beilinson-Kato class attached to $A$. According to Kato's reciprocity law [Kat04]

$$
\exp _{A}^{*}\left(\operatorname{res}_{p}\left(\zeta^{\mathrm{BK}}\right)\right)=\left(1-\frac{1}{p}\right) \frac{L(A / \mathbf{Q}, 1)}{\Omega_{A}^{+}} \in \mathbf{Q},
$$

where $\Omega_{A}^{+} \in \mathbf{R}^{*}$ is the real Néron period of $A$ and $\exp _{A}^{*}: H^{1}\left(\mathbf{Q}_{p}, V_{p}(A)\right) \rightarrow \operatorname{Fil}^{0} D_{\mathrm{dR}}\left(V_{p}(A)\right) \cong \mathbf{Q}_{p}$ is the BlochKato dual exponential map (see Section 2.6 for the last isomorphism). In particular this implies that the complex Hasse-Weil $L$-function $L(A / \mathbf{Q}, s)$ vanishes at $s=1$ precisely if $\zeta^{\mathrm{BK}}$ is a Selmer class, i.e. if it belongs to the Bloch-Kato Selmer group $H_{f}^{1}\left(\mathbf{Q}, V_{p}(A)\right) \subset H^{1}\left(\mathbf{Q}, V_{p}(A)\right)$ of $V_{p}(A)$.

When $L(A / \mathbf{Q}, 1)=0$, it is natural to ask whether $\zeta^{\mathrm{BK}}$ is still related to the special values of $L(A / \mathbf{Q}, s)$. Perrin-Riou addresses this question in [PR93] for elliptic curves with good reduction at $p$. In that setting, she conjectures that the logarithm of the $p$-adic Beilinson-Kato class equals the square of the logarithm of a Heegner point on the elliptic curve, up to a non-zero rational factor. In particular, she predicts that the Beilinson-Kato class is non-zero precisely if the Hasse-Weil $L$-function has a simple zero at $s=1$. The first aim of this paper is to prove the analogue of Perrin-Riou's conjecture in our multiplicative setting. 
Since $A / \mathbf{Q}_{p}$ is split multiplicative, Tate's theory provides a $G_{\mathbf{Q}_{p}}$-equivariant $p$-adic uniformisation

$$
\Phi_{\text {Tate }}: \overline{\mathbf{Q}}_{p}^{*} / q_{A}^{\mathbf{Z}} \cong A\left(\overline{\mathbf{Q}}_{p}\right),
$$

where $q_{A} \in p \mathbf{Z}_{p}$ is the Tate period of $A / \mathbf{Q}_{p}$. Denote by $\log _{q_{A}}: \mathbf{Q}_{p}^{*} / q_{A}^{\mathbf{Z}} \rightarrow \mathbf{Q}_{p}$ the branch of the $p$-adic logarithm which vanishes at $q_{A}$ and by

$$
\log _{A}=\log _{q_{A}} \circ \Phi_{\text {Tate }}^{-1}: A\left(\mathbf{Q}_{p}\right) \longrightarrow \mathbf{Q}_{p}
$$

the formal group logarithm on $A / \mathbf{Q}_{p}$. It induces an isomorphism $\log _{A}: A\left(\mathbf{Q}_{p}\right) \widehat{\otimes} \mathbf{Q}_{p} \cong \mathbf{Q}_{p}$ on $p$-adic completions.

Theorem A. Assume that $L(A / \mathbf{Q}, 1)=0$, i.e. that $\zeta^{\mathrm{BK}}$ is a Selmer class.

1. There exist a non-zero rational number $\ell_{1} \in \mathbf{Q}^{*}$ and a rational point $\mathbf{P} \in A(\mathbf{Q}) \otimes \mathbf{Q}$ such that

$$
\log _{A}\left(\operatorname{res}_{p}\left(\zeta^{\mathrm{BK}}\right)\right)=\ell_{1} \cdot \log _{A}^{2}(\mathbf{P}) .
$$

2. $\mathbf{P}$ is non-zero if and only if $L(A / \mathbf{Q}, s)$ has a simple zero at $s=1$.

In particular: $\operatorname{res}_{p}\left(\zeta^{\mathrm{BK}}\right) \neq 0$ if and only if $L(A / \mathbf{Q}, s)$ has a simple zero at $s=1$.

The point $\mathbf{P} \in A(\mathbf{Q}) \otimes \mathbf{Q}$ which appears in the statement is a Heegner point, coming from a certain Shimura curve parametrisation of $A$ (see Section 2.5). Theorem A then compares two Euler systems of a different nature: Kato's Euler system, belonging to the cyclotomic Iwasawa theory of $A$, and the Euler system of Heegner points, which pertains to the anticyclotomic Iwasawa theory of $A$ (and a suitable quadratic imaginary field).

The proof of Theorem A relies on Hida's theory of $p$-adic families of modular forms. Together with the work of Kato and Coleman-Perrin-Riou mentioned above, the exceptional zero formula proved by Bertolini and Darmon in [BD07], and Nekovář's theory of Selmer complexes [Nek06] are the key ingredients in our proof.

Remark. 1. Assume that $L(A / \mathbf{Q}, s)$ has a simple zero at $s=1$. By the theorem of Gross-Zagier-Kolyvagin, $A(\mathbf{Q})$ has rank one and $A(\mathbf{Q}) \otimes \mathbf{Q}_{p}=H_{f}^{1}\left(\mathbf{Q}, V_{p}(A)\right)$ is generated by $\mathbf{P}$. By Theorem $\mathrm{A}, \zeta^{\text {BK }}$ is equal to $\log _{A}(\mathbf{P}) \cdot \mathbf{P}$, up to a non-zero rational factor. According to [Ber77, Corollaire 2], $\log _{A}(\mathbf{P}) \in \mathbf{Q}_{p}^{*}$ is transcendental over $\mathbf{Q}$, so that $\zeta^{\mathrm{BK}} \notin A(\mathbf{Q}) \otimes \overline{\mathbf{Q}}$. In particular, $\zeta^{\mathrm{BK}}$ does not come from a rational point in $A(\mathbf{Q}) \otimes \mathbf{Q}$.

2. Bertolini and Darmon have recently announced [BD14] a proof of Perrin-Riou's conjecture for elliptic curves with good ordinary reduction at $p$. Their approach, based on the $p$-adic Beilinson formula proved in loc. cit. and the $p$-adic Gross-Zagier formula proved in [BDP13], is markedly different from ours.

Combining Theorem A, the results of Kato and Kolyvagin's method, we deduce the following result.

THEOREM B. $\zeta^{\mathrm{BK}}$ is non-zero if and only if $\operatorname{ord}_{s=1} L(A / \mathbf{Q}, s) \leq 1$.

1.2. $p$-adic Gross-Zagier formulae. Let $\chi_{\text {cyc }}: G_{\infty} \cong 1+p \mathbf{Z}_{p}$ denote the $p$-adic cyclotomic character. For every $s \in \mathbf{Z}_{p}$, set $L_{p}(A / \mathbf{Q}, s):=\chi_{\text {cyc }}^{s-1}\left(L_{p}(A / \mathbf{Q})\right)$. Then $L_{p}(A / \mathbf{Q}, s)$ is a $p$-adic analytic function on $\mathbf{Z}_{p}$. Since $A$ has split multiplicative reduction at $p$, the phenomenon of exceptional zeros discovered in [MTT86] implies that $L_{p}(A / \mathbf{Q}, 1)=0$ independently of whether $L(A / \mathbf{Q}, s)$ vanishes or not at $s=1$. The exceptional zero conjecture formulated in loc. cit. states that $\operatorname{ord}_{s=1} L_{p}(A / \mathbf{Q}, s)=\operatorname{ord}_{s=1} L(A / \mathbf{Q}, s)+1$, and that the leading term in the Taylor expansion of $L_{p}(A / \mathbf{Q}, s)$ at $s=1$ equals, up to a non-zero rational factor, the determinant of the lattice $A^{\dagger}(\mathbf{Q}) /$ torsion, computed with respect to the extended cyclotomic $p$-adic height pairing. Here $A^{\dagger}(\mathbf{Q})$ is the extended Mordell-Weil group, whose elements are pairs $\left(P, y_{P}\right) \in A(\mathbf{Q}) \times \mathbf{Q}_{p}^{*}$ such that $\Phi_{\text {Tate }}\left(y_{P}\right)=P$; it is an extension of $A(\mathbf{Q})$ by the $\mathbf{Z}$-module generated by the Tate period $q_{A}=\left(0, q_{A}\right) \in A^{\dagger}(\mathbf{Q})$. When $L(A / \mathbf{Q}, 1) \neq 0$ the conjecture predicts

$$
\frac{d}{d s} L_{p}(A / \mathbf{Q}, s)_{s=1}=\mathscr{L}_{p}(A) \frac{L(A / \mathbf{Q}, 1)}{\Omega_{A}^{+}},
$$

where $\mathscr{L}_{p}(A)=\log _{p}\left(q_{A}\right) / \operatorname{ord}_{p}\left(q_{A}\right)$ is the $\mathscr{L}$-invariant of $A / \mathbf{Q}_{p}$. This formula was proved by Greenberg and Stevens in [GS93]. (We give a slightly different proof of it in Theorem 5.2 below.)

Our second aim in this paper is to prove (a large part of) the above exceptional zero conjecture when $\operatorname{ord}_{s=1} L(A / \mathbf{Q}, s)=1$ and, more generally, a two-variable $p$-adic Gross-Zagier formula for the Mazur-Kitagawa $p$ adic $L$-function of the Hida family attached to $A / \mathbf{Q}$. Let $f \in S_{2}\left(\Gamma_{0}(N p), \mathbf{Z}\right)$ be the weight-two newform associated with $A / \mathbf{Q}$ by the modularity theorem, and let $f_{\infty}=\sum_{n=1}^{\infty} a_{n}(k) \cdot q^{n} \in \mathscr{A}_{U} \llbracket q \rrbracket$ be the Hida family passing through $f$. Here $U \subset \mathbf{Z}_{p}$ is a $p$-adic disc centred at 2 , and $\mathscr{A}_{U} \subset \mathbf{Q}_{p} \llbracket k-2 \rrbracket$ is the subring of power series in the variable $k-2$ which converge for $k \in U$. For every $k \in U \cap \mathbf{Z}^{\geq 2}$, the $q$-expansion $f_{k}:=\sum_{n=1}^{\infty} a_{n}(k) \cdot q^{n} \in S_{k}\left(\Gamma_{1}(N p), \mathbf{Z}_{p}\right)$ is an $N$-new $p$-ordinary Hecke eigenform of weight $k$, and $f_{2}=f$ (cf. Section 2.4). Thanks to the work of MazurKitagawa [Kit94] and Greenberg-Stevens [GS93], the $p$-adic $L$-functions of the forms $f_{k}$, for $k \in U \cap \mathbf{Z}^{\geq 2}$, can be packaged into a single two-variable $p$-adic $L$-function $L_{p}\left(f_{\infty}, k, s\right) \in \mathscr{A}$, where $\mathscr{A} \subset \mathbf{Q}_{p} \llbracket k-2, s-1 \rrbracket$ is the ring of formal power series converging for every $(k, s) \in U \times \mathbf{Z}_{p}$ (cf. Section 2.4). In particular one has $L_{p}\left(f_{\infty}, 2, s\right)=L_{p}(A / \mathbf{Q}, s)$ and the exceptional zero phenomenon implies that $L_{p}\left(f_{\infty}, k, s\right) \in \mathscr{J}$, where $\mathscr{J} \subset \mathscr{A}$ is the ideal of functions vanishing at $(k, s)=(2,1)$. 
Let $\widetilde{H}_{f}^{1}\left(\mathbf{Q}, V_{p}(A)\right)$ be Nekovár̆'s extended Selmer group. It is a $\mathbf{Q}_{p}$-module, equipped with a natural inclusion $A^{\dagger}(\mathbf{Q}) \otimes \mathbf{Q}_{p} \hookrightarrow \widetilde{H}_{f}^{1}\left(\mathbf{Q}, V_{p}(A)\right)$, which is an isomorphism precisely when the p-primary part of the Tate-Shafarevich group of $A / \mathbf{Q}$ is finite. In general $\widetilde{H}_{f}^{1}\left(\mathbf{Q}, V_{p}(A)\right)$ is canonically isomorphic to the direct sum of the Bloch-Kato Selmer group $H_{f}^{1}\left(\mathbf{Q}, V_{p}(A)\right)$ and the 1-dimensional vector space $\mathbf{Q}_{p} \cdot q_{A}$ generated by the Tate period of $A / \mathbf{Q}_{p}$ (see Section 4.2). Using Nekováŕ's results and ideas (especially [Nek06, Section 11]), we introduce in Section 4 a canonical $\mathbf{Q}_{p}$-bilinear form

$$
\langle-,-\rangle_{p}: \widetilde{H}_{f}^{1}\left(\mathbf{Q}, V_{p}(A)\right) \otimes_{\mathbf{Q}_{p}} \widetilde{H}_{f}^{1}\left(\mathbf{Q}, V_{p}(A)\right) \longrightarrow \mathscr{J} / \mathscr{J}^{2},
$$

called the cyclotomic height-weight pairing. One can write

$$
\langle-,-\rangle_{p}=\langle-,-\rangle_{p}^{\mathrm{cyc}} \cdot\{s-1\}+\langle-,-\rangle_{p}^{\mathrm{wt}} \cdot\{k-2\},
$$

where $\langle-,-\rangle_{p}^{\text {cyc }}$ and $\langle-,-\rangle_{p}^{\text {wt }}$ are canonical $\mathbf{Q}_{p}$-valued pairings on $\widetilde{H}_{f}^{1}\left(\mathbf{Q}, V_{p}(A)\right)$ and $\{\cdot\}: \mathscr{J} \rightarrow \mathscr{J} / \mathscr{J}^{2}$ denotes the projection. It turns out that the restriction

$$
\langle-,-\rangle_{p}^{\text {cyc }}: H_{f}^{1}\left(\mathbf{Q}, V_{p}(A)\right) \otimes_{\mathbf{Q}_{p}} H_{f}^{1}\left(\mathbf{Q}, V_{p}(A)\right) \longrightarrow \mathbf{Q}_{p}
$$

of $\langle-,-\rangle_{p}^{c y c}$ to the Bloch-Kato Selmer group is the cyclotomic canonical $p$-adic height pairing, as defined, e.g. in [Nek93, Section 7] (see Section 4.3.3 for more details). On the other hand, the weight pairing $\langle-,-\rangle_{p}^{\mathrm{wt}}$ is intrinsically associated with Hida's $p$-ordinary deformation of $T_{p}(A)$ (cf. Section 2.2). For every Selmer class $x \in H_{f}^{1}\left(\mathbf{Q}, V_{p}(A)\right)$, define its extended p-adic height-weight

$$
\widetilde{h}_{p}(x):=\operatorname{det}\left(\begin{array}{cc}
\left\langle q_{A}, q_{A}\right\rangle_{p} & \left\langle\left\langle q_{A}, x\right\rangle_{p}\right. \\
\left\langle x, q_{A}\right\rangle_{p} & \left\langle\langle x, x\rangle_{p}\right.
\end{array}\right) \in \mathscr{J}^{2} / \mathscr{J}^{3} .
$$

Let $\operatorname{sign}(A / \mathbf{Q}) \in\{ \pm 1\}$ be the sign in the functional equation of $L(A / \mathbf{Q}, s)$, and consider the condition

$($ Loc $) L(A / \mathbf{Q}, 1)=0$ and the restriction map $\operatorname{res}_{p}: H_{f}^{1}\left(\mathbf{Q}, V_{p}(A)\right) \rightarrow A\left(\mathbf{Q}_{p}\right) \widehat{\otimes} \mathbf{Q}_{p}$ is non-zero.

The work of Gross-Zagier-Kolyvagin guarantees that this condition is satisfied when $A(\mathbf{Q})$ is infinite and (in particular) when $L(A / \mathbf{Q}, s)$ has a simple zero at $s=1$. We can finally state the two-variable $p$-adic Gross-Zagier formula mentioned above.

Theorem C. Assume that $\operatorname{sign}(A / \mathbf{Q})=-1$ and that $(\mathbf{L o c})$ holds true. Let $\mathbf{P} \in A(\mathbf{Q}) \otimes \mathbf{Q}$ be as in Theorem A. Then $L_{p}\left(f_{\infty}, k, s\right) \in \mathscr{J}^{2}$ and there exists a non-zero rational number $\ell_{2} \in \mathbf{Q}^{*}$ such that

$$
L_{p}\left(f_{\infty}, k, s\right) \bmod \mathscr{J}^{3}=\ell_{2} \cdot \widetilde{h}_{p}(\mathbf{P}) .
$$

Moreover, $L_{p}\left(f_{\infty}, k, s\right) \in \mathscr{J}^{3}$ if and only if $\mathbf{P}=0$ (i.e. $L(A / \mathbf{Q}, s)$ vanishes to order greater than one at $s=1$ ).

1.2.1. Application to the exceptional zero conjecture. Recalling that $\log _{p}\left(q_{A}\right) \neq 0$ by [BSDGP96], define the Schneider height

$$
\langle-,-\rangle_{p}^{\mathrm{Sch}}: H_{f}^{1}\left(\mathbf{Q}, V_{p}(A)\right) \otimes_{\mathbf{Q}_{p}} H_{f}^{1}\left(\mathbf{Q}, V_{p}(A)\right) \longrightarrow \mathbf{Q}_{p}
$$

as the symmetric, $\mathbf{Q}_{p}$-bilinear form which for $x, y \in H_{f}^{1}\left(\mathbf{Q}, V_{p}(A)\right)$ is given by the formula

$$
\langle x, y\rangle_{p}^{\mathrm{Sch}}:=\langle x, y\rangle_{p}^{\mathrm{cyc}}-\frac{\log _{A}\left(\operatorname{res}_{p}(x)\right) \cdot \log _{A}\left(\operatorname{res}_{p}(y)\right)}{\log _{p}\left(q_{A}\right)} .
$$

The terminology is justified by the fact that $\langle-,-\rangle_{p}^{\text {Sch }}$ is the norm-adapted height constructed in [Sch82] (cf. Section 7.14 of [Nek06] and Chapter II, $\S 6$ of [MTT86]). As a consequence of Theorem C and the properties of $\left\langle\langle-,-\rangle_{p}\right.$, one deduces the following $p$-adic Gross-Zagier formula for $L_{p}(A / \mathbf{Q}, s)$, predicted by Conjecture $B S D(p)$ exceptional case in [MTT86, Chapter II, §10].

Theorem D. Assume that (Loc) holds true and let $\mathbf{P} \in A(\mathbf{Q}) \otimes \mathbf{Q}$ be as in Theorem A. Then $L_{p}(A / \mathbf{Q}, s)$ vanishes to order at least 2 at $s=1$, and there exists a non-zero rational number $\ell_{3} \in \mathbf{Q}^{*}$ such that

$$
\frac{d^{2}}{d s^{2}} L_{p}(A / \mathbf{Q}, s)_{s=1}=\ell_{3} \cdot \mathscr{L}_{p}(A) \cdot\langle\mathbf{P}, \mathbf{P}\rangle_{p}^{\mathrm{Sch}} .
$$

The preceding result enriches our repertoire of $p$-adic Gross-Zagier formulae for cyclotomic and anticyclotomic $p$-adic $L$-functions of elliptic curves, which already includes the main results of [PR87], [BD98] and [Kob13].

As $\mathscr{L}_{p}(A) \neq 0$, Theorem D implies that $\operatorname{ord}_{s=1} L_{p}(A / \mathbf{Q}, s)=2$ precisely if $\operatorname{ord}_{s=1} L(A / \mathbf{Q}, s)=1$ and $\langle-,-\rangle_{p}^{\text {Sch }}$ is non-zero. On the other hand, it is not known that the Schneider height is non-zero when $L(A / \mathbf{Q}, s)$ has a simple zero at $s=1$. 
1.2.2. The derivative of the improved $p$-adic L-function. As explained in [GS93], the restriction of $L_{p}\left(f_{\infty}, k, s\right)$ to the vertical line $s=1$ admits a factorisation $L_{p}\left(f_{\infty}, k, 1\right)=\left(1-a_{p}(k)^{-1}\right) \cdot L_{p}^{*}\left(f_{\infty}, k\right)$ in $\mathscr{A}_{U}$. The results of [GS93] and [BSDGP96] imply that the function $1-a_{p}(k)^{-1}$ has a simple zero at $k=2$. The following $p$-adic Gross-Zagier formula for the improved $p$-adic L-function $L_{p}^{*}\left(f_{\infty}, k\right)$ is again a consequence of Theorem $\mathrm{C}$ and the properties of the height-weight pairing.

Theorem E. Assume that hypothesis (Loc) holds and that $\operatorname{sign}(A / \mathbf{Q})=-1$. Let $\mathbf{P} \in A(\mathbf{Q}) \otimes \mathbf{Q}$ be as in Theorem $A$. Then $L_{p}^{*}\left(f_{\infty}, 2\right)=0$ and there exists a non-zero rational number $\ell_{4} \in \mathbf{Q}^{*}$ such that

$$
-\ell_{4} \cdot\langle\mathbf{P}, \mathbf{P}\rangle_{p}^{\mathrm{cyc}}=\frac{d}{d k} L_{p}^{*}\left(f_{\infty}, k\right)_{k=2}=2 \ell_{4} \cdot\langle\mathbf{P}, \mathbf{P}\rangle_{p}^{\mathrm{wt}}
$$

1.3. Outline of the proofs. We briefly sketch the strategy of the proofs of Theorems A and C, assuming for simplicity that $L(A / \mathbf{Q}, s)$ has a simple zero at $s=1$.

Denote by $L_{p}^{\mathrm{cc}}\left(f_{\infty}, k\right):=L_{p}\left(f_{\infty}, k, k / 2\right) \in \mathscr{A}_{U}$ the restriction of $L_{p}\left(f_{\infty}, k, s\right)$ to the central critical line $s=k / 2$. According to the exceptional zero formula proved by Bertolini-Darmon in [BD07], $L_{p}^{\mathrm{cc}}\left(f_{\infty}, k\right)$ has order of vanishing 2 at $k=2$ and

$$
\frac{d^{2}}{d k^{2}} L_{p}^{\mathrm{cc}}\left(f_{\infty}, k\right)_{k=2}=\ell \cdot \log _{A}^{2}(\mathbf{P}),
$$

where $\ell \in \mathbf{Q}^{*}$ and $\mathbf{P} \in A(\mathbf{Q}) \otimes \mathbf{Q}$ is a Heegner point. (See Section 2.5 for more details.)

On the algebraic side, write $\widetilde{h}_{p}^{\text {cc }}: H_{f}^{1}\left(\mathbf{Q}, V_{p}(A)\right) \rightarrow \mathbf{Q}_{p}$ for the composition of the extended height-weight $\widetilde{h}_{p}$ with the morphism $\mathscr{J}^{2} / \mathscr{J}^{3} \rightarrow \mathbf{Q}_{p}$ which on the class of $\alpha(k, s) \in \mathscr{J}^{2}$ takes the value $\frac{d^{2}}{d k^{2}} \alpha(k, k / 2)_{k=2}$. The properties satisfied by the height-weight pairing (cf. Theorem 4.2) yield

$$
\widetilde{h}_{p}^{\mathrm{cc}}(x)=\frac{1}{2} \log _{A}^{2}\left(\operatorname{res}_{p}(x)\right)
$$

for every Selmer class $x$. Equation (5) can then be rephrased as the $p$-adic Gross-Zagier formula

$$
\frac{d^{2}}{d k^{2}} L_{p}^{\mathrm{cc}}\left(f_{\infty}, k\right)_{k=2}=2 \ell \cdot \widetilde{h}_{p}^{\mathrm{cc}}(\mathbf{P})
$$

This shows that the formula displayed in Theorem $\mathrm{C}$ holds true, once one restricts both $L_{p}\left(f_{\infty}, k, s\right)$ and $\widetilde{h}_{p}(\mathbf{P})$ to the central critical line $s=k / 2$. Instead of trying to extend $(7)$ to the $(k, s)$-plane directly, we first prove an analogue of Theorem $\mathbf{C}$, in which the Heegner point $\mathbf{P}$ is replaced by the Beilinson-Kato class $\zeta^{\mathrm{BK}}$. Precisely, making use of the work of Kato and Ochiai, we prove in Section 5 the equality in $\mathscr{J}^{2} / \mathscr{J}^{3}$ :

$$
\log _{A}\left(\operatorname{res}_{p}\left(\zeta^{\mathrm{BK}}\right)\right) \cdot L_{p}\left(f_{\infty}, k, s\right) \bmod \mathscr{J}^{3}=\frac{-1}{\operatorname{ord}_{p}\left(q_{A}\right)}\left(1-\frac{1}{p}\right)^{-1} \cdot \widetilde{h}_{p}\left(\zeta^{\mathrm{BK}}\right) .
$$

Combined with (5) and (6), this gives

$$
\log _{A}^{2}\left(\operatorname{res}_{p}\left(\zeta^{\mathrm{BK}}\right)\right)=\ell_{1} \cdot \log _{A}^{2}(\mathbf{P}) \cdot \log _{A}\left(\operatorname{res}_{p}\left(\zeta^{\mathrm{BK}}\right)\right),
$$

where $\ell_{1}:=-2 \ell \cdot \operatorname{ord}_{p}\left(q_{A}\right)\left(1-p^{-1}\right)$. We then show that $\operatorname{res}_{p}\left(\zeta^{\mathrm{BK}}\right) \neq 0$ and deduce Theorem A. Now, thanks to the theorem of Gross-Zagier-Kolyvagin, one has $\zeta^{\mathrm{BK}}=\lambda \cdot \mathbf{P}$, with $\lambda=\log _{A}\left(\operatorname{res}_{p}\left(\zeta^{\mathrm{BK}}\right)\right) / \log _{A}(\mathbf{P}) \in \mathbf{Q}_{p}^{*}$. Then $\widetilde{h}_{p}\left(\zeta^{\mathrm{BK}}\right)=\lambda^{2} \cdot \widetilde{h}_{p}(\mathbf{P})$. If one sets $\ell_{2}:=2 \ell$, Theorem A and equation (8) yield Theorem $\mathrm{C}$, namely

$$
L_{p}\left(f_{\infty}, k, s\right) \bmod \mathscr{J}^{3}=\ell_{2} \cdot \widetilde{h}_{p}(\mathbf{P}) .
$$

Organisation of the paper. Section 2 recalls the known results needed in the rest of the paper. This includes some basic facts from Hida's theory, the main result of [BD07] mentioned above, Ochiai's construction of a two variable big dual exponential and a general version of Kato's reciprocity law. In Section 3 we compute the derivative of Ochiai's big dual exponential. Section 4 introduces the height-weight pairing $\langle-,-\rangle_{p}$ and discusses its basic properties. In Section 5, we use the computations carried out in Section 3 to prove certain exceptional zero Rubin's formulae, relating the big dual exponential and the height-weight pairing. Combining these formulae with Kato's work, we are able to prove a variant of the main result of [GS93] and to prove the key equality (8) appearing above. Finally, in Section 6 we prove the results stated above.

Acknowledgements. Much of the work on this article was carried out during my Ph.D. at the University of Milan. It is a pleasure to express my sincere gratitude to my supervisor, Prof. Massimo Bertolini, who constantly encouraged and motivated my work. Every meeting with him has been a source of ideas and enthusiasm; this paper surely originated from and grew up through these meetings. I would like to thank Marco Seveso for a careful reading of the paper and for many interesting discussions related to this work. I am also grateful to the anonymous referee; the current version of the article is greatly inspired by his/her corrections and valuable comments, which helped me to significantly clarify and improve the exposition. 


\section{Hida families, exceptional zeros and Euler systems}

2.1. The Hida family. Set $\Gamma:=1+p \mathbf{Z}_{p}$ and $\Lambda:=\mathbf{Z}_{p} \llbracket \Gamma \rrbracket$. Let $C$ be a finite, flat $\Lambda$-algebra. A continuous $\mathbf{Z}_{p}$-algebra morphism $\nu: C \rightarrow \overline{\mathbf{Q}}_{p}$ is an arithmetic point of weight $k$ and character $\chi$ if its restriction to $\Gamma$ under the structural morphism is of the form $\gamma \mapsto \gamma^{k-2} \cdot \chi(\gamma)$, for an integer $k \geq 2$ and a character $\chi: \Gamma \rightarrow \overline{\mathbf{Q}}_{p}^{*}$ of finite order. Denote by $\mathcal{X}^{\text {arith }}(C)$ the set of arithmetic points of $C$.

Let $f=\sum_{n=1}^{\infty} a_{n}(A) \cdot q^{n} \in S_{2}\left(\Gamma_{0}(N p), \mathbf{Z}\right)$ be the weight-two newform attached to $A / \mathbf{Q}$ by the modularity theorem of Wiles, Taylor-Wiles et alii. According to the work of Hida [Hid86b], [Hid86a] there exists an R-adic eigenform of tame level $N$ :

$$
\mathbf{f}=\sum_{n=1}^{\infty} \mathbf{a}_{n} \cdot q^{n} \in R \llbracket q \rrbracket
$$

passing through $f$. Here $R=R_{f}$ is a normal local Noetherian domain, finite and flat over $\Lambda$, and $\mathbf{f}$ is a formal power series with coefficients in $R$ satisfying the following properties. For every arithmetic point $\nu \in \mathcal{X}^{\operatorname{arith}}(R)$ of weight $k \geq 2$ and character $\chi$, the $\nu$-specialisation

$$
f_{\nu}:=\sum_{n=1}^{\infty} \nu\left(\mathbf{a}_{n}\right) \cdot q^{n} \in S_{k}\left(\Gamma_{0}\left(N p^{r}\right), \chi \omega^{2-k}\right)
$$

is the $q$-expansion of an $N$-new $p$-ordinary Hecke eigenform of level $N p^{r}$, weight $k$, and character $\chi \cdot \omega^{2-k}$. Here $r$ is the smallest positive integer such that $1+p^{r} \mathbf{Z}_{p} \subset \operatorname{ker}(\chi)$ and $\omega$ is the Teichmüller character. Moreover, there exists a distinguished arithmetic point $\psi=\nu_{f} \in \mathcal{X}^{\text {arith }}(R)$ of weight 2 and trivial character such that

$$
f=f_{\psi} \text {. }
$$

With the notations of Section 1 of [Hid86a], let $h^{o}\left(N ; \mathbf{Z}_{p}\right)$ be the universal $p$-ordinary Hecke algebra of tame level $N$. Diamond operators give a morphism of $\mathbf{Z}_{p}$-algebras [.] $: \Lambda \rightarrow h^{o}\left(N ; \mathbf{Z}_{p}\right)$, making $h^{o}\left(N ; \mathbf{Z}_{p}\right)$ a free, finitely generated $\Lambda$-module [Hid86b, Theorem 3.1]. (We assume here that [.] is normalised as in Section 1.4 of [NP00].) The ring $R$, denoted $\mathscr{I}(\mathscr{K})$ in [Hid86a], is the integral closure of $\Lambda$ in the primitive component $\mathscr{K}=\mathscr{K}_{f}$ of $h^{o}\left(N ; \mathbf{Z}_{p}\right) \otimes_{\Lambda} \operatorname{Frac}(\Lambda)$ to which $f$ belongs [Hid86a, Corollary 1.3].

Let $\nu \in \mathcal{X}^{\operatorname{arith}}(R)$. By [Hid86a, Corollary 1.4] the localisation of $R$ at the kernel of $\nu$ is a discrete valuation ring, unramified over the localisation of $\Lambda$ at $\Lambda \cap \operatorname{ker}(\nu)$. In particular, fix a topological generator $\gamma_{0} \in \Gamma$, let $\varpi:=\gamma_{0}-1 \in \Lambda$ and write $\mathfrak{p}=\mathfrak{p}_{\psi}:=\operatorname{ker}(\psi)$. Then

$$
\mathfrak{p} R_{\mathfrak{p}}=\varpi \cdot R_{\mathfrak{p}},
$$

i.e. $\varpi$ is a prime element of $R_{\mathfrak{p}}$.

2.2. Hida's $R$-adic representation. Let $\mathbb{T}=\mathbb{T}_{\mathbf{f}}$ be the $p$-ordinary $R$-adic representation attached by Hida to $\mathbf{f}$ in [Hid86a, Theorem 2.1]. More precisely, let $J_{\infty}^{o}\left[p^{\infty}\right]$ be the 'big' $p$-divisible group appearing in Section 8 of [Hid86a], which is a $h^{o}\left(N ; \mathbf{Z}_{p}\right)$-module of co-finite rank. We define $\mathbb{T}:=\operatorname{Hom}_{\mathbf{Z}_{p}}\left(J_{\infty}^{o}\left[p^{\infty}\right], \mu_{p^{\infty}}\right) \otimes_{h^{o}\left(N ; \mathbf{Z}_{p}\right)} R$. It is a rank-two $R$-module, equipped with a continuous $R$-linear action of $G_{\mathbf{Q}}$, which is unramified at every rational prime $l \nmid N p$. According to Théorème 7 of [MT90] our assumption on the irreducibility of $A_{p}$ implies that $\mathbb{T}$ is a free $R$-module of rank two and that

$$
\operatorname{Trace}\left(\operatorname{Frob}_{l} \mid \mathbb{T}\right)=\mathbf{a}_{l} ; \quad \operatorname{det}\left(\operatorname{Frob}_{l} \mid \mathbb{T}\right)=l[l]
$$

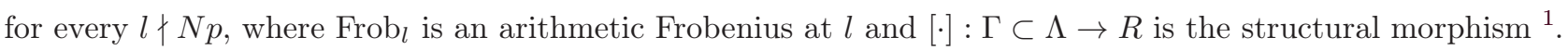

2.2.1. Ramification at $p$. Let $G_{p}:=G_{\mathbf{Q}_{p}} \hookrightarrow G_{\mathbf{Q}}$ be the decomposition group determined by our choice of $i_{p}: \overline{\mathbf{Q}} \hookrightarrow \overline{\mathbf{Q}}_{p}$ and let $I_{p}:=I_{\mathbf{Q}_{p}}$ be its inertia subgroup. By loc. cit. (see also [NP00, Section 1.5]) there exists an exact sequence of $R\left[G_{p}\right]$-modules

$$
0 \longrightarrow \mathbb{T}^{+} \stackrel{i^{+}}{\longrightarrow} \mathbb{T} \stackrel{p^{-}}{\longrightarrow} \mathbb{T}^{-} \longrightarrow 0
$$

where $\mathbb{T}^{+}$and $\mathbb{T}^{-}$are free $R$-modules of rank 1 and $\mathbb{T}^{-}$is unramified. Moreover, write $\widetilde{\mathbf{a}}_{p}: G_{p} \rightarrow G_{p} / I_{p} \rightarrow R^{*}$ for the unramified character sending the arithmetic Frobenius $\operatorname{Frob}_{p} \in G_{p} / I_{p}$ to the $p$-th Hecke operator $\mathbf{a}_{p}$. Then $G_{p}$ acts on $\mathbb{T}^{-}$via $\widetilde{\mathbf{a}}_{p}$ and on $\mathbb{T}^{+}$via $\widetilde{\mathbf{a}}_{p}^{-1} \chi_{\text {cyc }}\left[\kappa_{\text {cyc }}\right]$, i.e.

$$
\mathbb{T}^{+} \cong R\left(\chi_{\mathrm{cyc}}\left[\kappa_{\mathrm{cyc}}\right] \widetilde{\mathbf{a}}_{p}^{-1}\right) ; \quad \mathbb{T}^{-} \cong R\left(\widetilde{\mathbf{a}}_{p}\right) .
$$

As in the introduction, $\chi_{\mathrm{cyc}}: G_{\mathbf{Q}} \rightarrow \mathbf{Z}_{p}^{*}$ is the $p$-adic cyclotomic character, and $\kappa_{\mathrm{cyc}}: G_{\mathbf{Q}} \rightarrow \Gamma$ is the composition of $\chi_{\mathrm{cyc}}$ with the projection to principal units.

\footnotetext{
${ }^{1}$ Théorème 7 of [MT90] proves these facts assuming that the residual Galois representation $\bar{\rho}_{\mathbf{f}}$ of $\mathbb{T}$ is absolutely irreducible. As pointed out to us by J. Nekovář, loc. cit. also requires $\bar{\rho}_{\mathbf{f}}$ to be $p$-distinguished (see $[\mathbf{E P W 0 6}]$ ). As $\bar{\rho}_{\mathbf{f}} \cong A_{p}$ and $p \neq 2$, this hypothesis is automatically satisfied in our case, by Tate's theory of $p$-adic uniformisation.
} 
2.2.2. Specialisations. Let $\nu \in \mathcal{X}^{\operatorname{arith}}(R)$, let $K_{\nu}:=\operatorname{Frac}(\nu(R))$ and let $V_{\nu}$ be the contragredient of the $K_{\nu^{-}}$ adic Deligne representation of $G_{\mathbf{Q}}$ attached to the eigenform $f_{\nu}$. It follows from [Oht95, Theorem 1.4.3] that the representation $\mathbb{T}_{\nu}:=\mathbb{T} \otimes_{R, \nu} \nu(R)$ is canonically isomorphic to a Galois-stable $\nu(R)$-lattice in $V_{\nu}$; in particular there is a natural isomorphism

$$
\mathbb{T}_{\nu} \otimes \mathbf{z}_{p} \mathbf{Q}_{p} \cong V_{\nu}
$$

We identify from now on $\mathbb{T}_{\nu}$ with a Galois-stable $\nu(R)$-lattice in $V_{\nu}$.

Considering the arithmetic point $\psi \in \mathcal{X}^{\operatorname{arith}}(R)$ corresponding to $f$, one has $\mathbb{T}_{\psi} \otimes \mathbf{z}_{p} \mathbf{Q}_{p} \cong V_{p}(A)$. Indeed, the irreducibility of $A_{p}$ implies that $\psi$ induces a canonical isomorphism of $\mathbf{Z}_{p}\left[G_{\mathbf{Q}}\right]$-modules

$$
\pi_{f}: \mathbb{T}_{\psi} \cong T_{p}(A) \text {. }
$$

Recall the Tate parametrisation $\Phi_{\text {Tate }}$ introduced in (3). As $q_{A}$ has positive valuation, $\Phi_{\text {Tate }}$ induces on the $p$-adic Tate modules a short exact sequence of $\mathbf{Z}_{p}\left[G_{p}\right]$-modules

$$
0 \longrightarrow \mathbf{Z}_{p}(1) \stackrel{i^{+}}{\longrightarrow} T_{p}(A) \stackrel{p^{-}}{\longrightarrow} \mathbf{Z}_{p} \longrightarrow 0
$$

We also write $T_{p}(A)^{+}:=\mathbf{Z}_{p}(1)$ and $T_{p}(A)^{-}:=\mathbf{Z}_{p}$. By (11) there are isomorphisms of $G_{p^{-}}$modules

$$
\pi_{f}^{+}: \mathbb{T}_{\psi}^{+}:=\mathbb{T}^{+} \otimes_{R, \psi} \mathbf{Z}_{p} \cong \mathbf{Z}_{p}(1) ; \quad \pi_{f}^{-}: \mathbb{T}_{\psi}^{-}:=\mathbb{T}^{-} \otimes_{R, \psi} \mathbf{Z}_{p} \cong \mathbf{Z}_{p}
$$

We can, and will, normalise $\pi_{f}^{ \pm}$in such a way that they are compatible with $\pi_{f}$.

2.3. $p$-adic $L$-functions. Let $G_{\infty}$ and $\Lambda_{\text {cyc }}$ be as in the introduction, and define $\bar{R}:=R \llbracket G_{\infty} \rrbracket=R \widehat{\otimes}_{\mathbf{z}_{p}} \Lambda_{\text {cyc }}$. Under our assumptions Section 3.4 of [EPW06] (using ideas from [Kit94] and [GS93]) attaches to $\mathbf{f}$ an element

$$
L_{p}(\mathbf{f}) \in \bar{R},
$$

unique up to multiplication by units in $R$, which interpolates the Mazur-Tate-Teitelbaum $p$-adic $L$-functions of the arithmetic specialisations of $\mathbf{f}$. More precisely, given $\nu \in \mathcal{X}^{\operatorname{arith}}(R)$, let $\bar{R}_{\nu}:=\nu(R) \llbracket G_{\infty} \rrbracket$ and write again

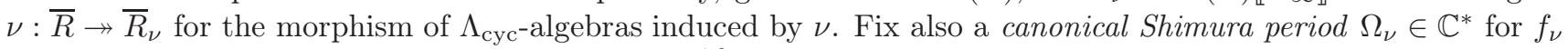
(see [EPW 06, Sec. 3.1]). Then, for every $\nu \in \mathcal{X}^{\operatorname{arith}}(R)$, there exists a scalar $\lambda_{\nu} \in \nu(R)^{*}$ such that

$$
\nu\left(L_{p}(\mathbf{f})\right)=\lambda_{\nu} \cdot L_{p}\left(f_{\nu}\right) \in \bar{R}_{\nu}
$$

where $L_{p}\left(f_{\nu}\right)=L_{p, \Omega_{\nu}}\left(f_{\nu}\right)$ is the Mazur-Tate-Teitelbaum $p$-adic $L$-function attached in [MTT86] to $f_{\nu}$, normalised with respect to $\Omega_{\nu}$ (see also [GS93, Section 4]). It is characterised by the following interpolation property: let $k_{\nu}$ be the weight of $\nu$. Then for every finite order character $\chi: G_{\infty} \rightarrow \overline{\mathbf{Q}}_{p}^{*}$ and every integer $0<s_{0}<k_{\nu}$

$$
\chi \cdot \chi_{\mathrm{cyc}}^{s_{0}-1}\left(L_{p}\left(f_{\nu}\right)\right)=\nu\left(\mathbf{a}_{p}\right)^{-m}\left(1-\frac{\chi \omega^{1-s_{0}}(p) \cdot p^{s_{0}-1}}{\nu\left(\mathbf{a}_{p}\right)}\right) L^{\operatorname{alg}}\left(f_{\nu}, \chi \omega^{1-s_{0}}, s_{0}\right),
$$

where $m$ is the $p$-adic valuation of the conductor of $\chi$ and

$$
L^{\operatorname{alg}}\left(f_{\nu}, \chi \omega^{1-s_{0}}, s_{0}\right):=\tau\left(\chi \omega^{1-s_{0}}\right) p^{m\left(s_{0}-1\right)}\left(s_{0}-1\right) ! \frac{L\left(f_{\nu}, \chi^{-1} \omega^{s_{0}-1}, s_{0}\right)}{(2 \pi i)^{s_{0}-1} \Omega_{\nu}} \in \overline{\mathbf{Q}} .
$$

For a Dirichlet character $\mu, \tau(\mu)$ is the Gauss sum of $\mu$ and $L\left(f_{\nu}, \mu, s\right)$ is the Hecke $L$-function of $f_{\nu}$ twisted by $\mu$.

According to [GV00, Sec. 3], under our assumptions we can choose $\Omega_{\psi}=\Omega_{A}^{+}$as the real Néron period of $A / \mathbf{Q}$, so that $L_{p}(A / \mathbf{Q}):=L_{p}\left(f_{\psi}\right)$ is the $p$-adic L-function of $A / \mathbf{Q}$. Here we insist to make this choice and to normalise $L_{p}(\mathbf{f})$ by requiring $\lambda_{\psi}=1$, i.e.

$$
\psi\left(L_{p}(\mathbf{f})\right)=L_{p}(A / \mathbf{Q}) .
$$

Then $L_{p}(\mathbf{f})$ is a well-defined element of $\bar{R}$ up to multiplication by units $\alpha \in R^{*}$ such that $\psi(\alpha)=1$.

2.3.1. Exceptional zeros. The $p$-adic multiplier

$$
E_{p}\left(\nu, \chi \cdot \chi_{\text {сус }}^{j}\right):=\left(1-\frac{\chi \omega^{-j}(p) \cdot p^{j}}{\nu\left(\mathbf{a}_{p}\right)}\right)
$$

which appears in the interpolation formula (16) is responsible for the phenomenon of exceptional zeros mentioned in the introduction (cf. [MTT86]). Indeed $\psi\left(\mathbf{a}_{p}\right)=a_{p}(A)=+1$ in our setting, and $E_{p}(\psi, 1)=0$. In particular, let $I=I_{\text {cyc }}$ be the augmentation ideal of $\Lambda_{\text {cyc }}$ and let $\overline{\mathfrak{p}}=(\mathfrak{p}, I)$ be the ideal of $\bar{R}$ generated by $I$ and $\mathfrak{p}$. Then

$$
L_{p}(\mathbf{f}) \in \overline{\mathfrak{p}} ; \quad L_{p}(A / \mathbf{Q}) \in I .
$$

2.3.2. The improved $p$-adic L-function. Let $\varepsilon: \bar{R} \rightarrow R$ be the augmentation map. By [EPW06, Remark 3.4.5] (generalising a result of [GS93]) there is a factorisation

$$
\varepsilon\left(L_{p}(\mathbf{f})\right)=\left(1-\mathbf{a}_{p}^{-1}\right) \cdot L_{p}^{*}(\mathbf{f})
$$

for an element $L_{p}^{*}(\mathbf{f}) \in R$ called the improved $p$-adic $L$-function of $\mathbf{f}$. 
2.4. The analytic Mellin transform. As explained in [GS93, Section 2.6] (see also [NP00, Section 1.4.7]), there exist a disc $U \subset \mathbf{Z}_{p}$ centred at 2 and a unique morphism of $\Lambda$-modules

$$
\mathrm{M}=\mathrm{M}_{f}: R \longrightarrow \mathscr{A}_{U}
$$

such that $\left.\mathrm{M}(r)\right|_{k=2}=\psi(r)$ for every $r \in R$. Here $\mathscr{A}_{U} \subset \mathbf{Q}_{p} \llbracket k-2 \rrbracket$ (see Section 1.2) is endowed with the structure of a $\Lambda$-algebra via the character $\Gamma \rightarrow \mathscr{A}_{U}$ which sends $\gamma \in \Gamma$ to the power series $\gamma^{k-2}:=\exp _{p}\left((k-2) \cdot \log _{p}(\gamma)\right)$. The morphism $\mathrm{M}$ is called the Mellin transform centred at $k=2$. For every $n \in \mathbf{N}$, set $a_{n}(k):=\mathrm{M}\left(\mathbf{a}_{n}\right)$ and define

$$
f_{\infty}:=\sum_{n=1}^{\infty} a_{n}(k) \cdot q^{n} \in \mathscr{A}_{U} \llbracket q \rrbracket .
$$

Let $\mathscr{A} \subset \mathbf{Q}_{p} \llbracket k-2, s-1 \rrbracket$ and $\mathscr{J} \subset \mathscr{A}$ be as in Section 1.2. Then $\mathscr{A}$ has a structure of $\Lambda_{\text {cyc-algebra, induced }}$ by the character $G_{\infty} \rightarrow \mathscr{A}$ mapping $g \in G_{\infty}$ to $\chi_{\mathrm{cyc}}(g)^{s-1}:=\exp _{p}\left((s-1) \cdot \log _{p}\left(\chi_{\mathrm{cyc}}(g)\right)\right)$. Moreover there exists a unique morphism of $\Lambda_{\text {cyc-algebras }}$

$$
\overline{\mathrm{M}}=\overline{\mathrm{M}}_{f}: \bar{R} \longrightarrow \mathscr{A}
$$

whose restriction to $R$ equals $\mathrm{M}$, called the Mellin transform centred at $(k, s)=(2,1)$. Define the Mazur-Kitagawa p-adic L-function of $f_{\infty}$ :

$$
L_{p}\left(f_{\infty}, k, s\right):=\overline{\mathrm{M}}\left(L_{p}(\mathbf{f})\right) \in \mathscr{J}
$$

as the Mellin transform of $L_{p}(\mathbf{f}) \in \bar{R}$. More precisely, it is a well-defined element of $\mathscr{A}$ up to multiplication by a nowhere-vanishing function $\alpha(k) \in \mathscr{A}_{U}$ such that $\alpha(2)=1$, and belongs to $\mathscr{J}$ by equation (18). In the introduction we defined $L_{p}(A / \mathbf{Q}, s):=\chi_{\text {cyc }}^{s-1}\left(L_{p}(A / \mathbf{Q})\right)=\overline{\mathrm{M}}\left(L_{p}(A / \mathbf{Q})\right)$, so that equation (17) gives

$$
L_{p}\left(f_{\infty}, 2, s\right)=L_{p}(A / \mathbf{Q}, s) .
$$

According to Theorem 5.15 of [GS93] $L_{p}\left(f_{\infty}, k, s\right)$ satisfies the functional equation

$$
\Lambda_{p}\left(f_{\infty}, k, s\right)=-\operatorname{sign}(A / \mathbf{Q}) \cdot \Lambda_{p}\left(f_{\infty}, k, k-s\right),
$$

where $\Lambda_{p}\left(f_{\infty}, k, s\right):=\langle N\rangle^{s / 2} \cdot L_{p}\left(f_{\infty}, k, s\right),\langle\cdot\rangle: \mathbf{Z}_{p}^{*} \rightarrow 1+p \mathbf{Z}_{p}$ denotes the projection to principal units and $\operatorname{sign}(A / \mathbf{Q}) \in\{ \pm 1\}$ is the sign in the functional equation satisfied by the Hasse-Weil $L$-function of $A / \mathbf{Q}$. Note that the central critical line $s=k / 2$ is the 'centre of symmetry' of the functional equation. In particular, when $\operatorname{sign}(A / \mathbf{Q})=+1, L_{p}\left(f_{\infty}, k, k / 2\right)$ vanishes identically.

Write $L_{p}^{*}\left(f_{\infty}, k\right):=\mathrm{M}\left(L_{p}^{*}(\mathbf{f})\right) \in \mathscr{A}_{U}$. As $\mathrm{M} \circ \varepsilon=\left.\overline{\mathrm{M}}(\cdot)\right|_{s=1}$, equation (19) gives a factorisation in $\mathscr{A}_{U}$ :

$$
L_{p}\left(f_{\infty}, k, 1\right)=\left(1-a_{p}(k)^{-1}\right) \cdot L_{p}^{*}\left(f_{\infty}, k\right) .
$$

The function $L_{p}^{*}\left(f_{\infty}, k\right)$ is called the improved p-adic L-function of $f_{\infty}$.

2.5. The Bertolini-Darmon exceptional zero formula. The following result has been proved in [BD07], assuming a mild technical condition subsequently removed in [Mok11, Section 6]. Denote by $L_{p}^{\mathrm{cc}}\left(f_{\infty}, k\right) \in \mathscr{A}_{U}$ the restriction of $L_{p}\left(f_{\infty}, k, s\right)$ to the central critical line $s=k / 2$.

TheOREM 2.1. There exist a non-zero rational number $\ell \in \mathbf{Q}^{*}$ and a rational point $\mathbf{P} \in A(\mathbf{Q}) \otimes \mathbf{Q}$ such that

$$
\frac{d^{2}}{d k^{2}} L_{p}^{\mathrm{cc}}\left(f_{\infty}, k\right)_{k=2}=\ell \cdot \log _{A}^{2}(\mathbf{P}) .
$$

Moreover, $\mathbf{P}$ is non-zero if and only if $L(A / \mathbf{Q}, s)$ has a simple zero at $s=1$.

REmark 2.2. Assume for simplicity that $\operatorname{sign}(A / \mathbf{Q})=-1$ and that $N \neq 1$ is not square-full (see [Mok11] for the general case). As explained in [BD07], the definitions of $\mathbf{P}$ and $\ell$ rest on the choice of an auxiliary imaginary quadratic field $K / \mathbf{Q}$ satisfying the following conditions. Let $D_{K}$ and $\epsilon_{K}:\left(\mathbf{Z} / D_{K} \mathbf{Z}\right)^{*} \rightarrow\{ \pm 1\}$ denote the discriminant and the quadratic character of $K$ respectively.

( $\alpha)\left(D_{K}, N p\right)=1$ and there is a factorisation $N p=p N^{+} N^{-}$, such that $p N^{-}$is square-free and a prime divisor of $N p$ divides $p N^{-}$if and only if it is inert in $K$.

( $\beta)$ The special value $L\left(A / \mathbf{Q}, \epsilon_{K}, 1\right)$ is non-zero.

Then $\mathbf{P}$ is defined as the trace to $\mathbf{Q}$ of a Heegner point in $A(K) \otimes \mathbf{Q}$, coming from a parametrisation of $A / \mathbf{Q}$ by the Shimura curve $X_{N^{+}, p N^{-}}$associated with an Eichler order of level $N^{+}$in the indefinite quaternion algebra of discriminant $p N^{-}$. The rational number $\ell$ is defined by the relation

$$
2 \ell^{-1}=\eta_{f} \cdot \sqrt{D_{K}} \cdot \frac{L\left(A / \mathbf{Q}, \epsilon_{K}, 1\right)}{\Omega_{A}^{-}} \in \mathbf{Q}^{*} .
$$

Here $\Omega_{A}^{-} \in i \mathbf{R}^{*}$ is such that $\Omega_{A}^{+} \cdot \Omega_{A}^{-}$is the Petersson norm of $f$. The constant $\eta_{f}:=\left\langle\phi_{f}, \phi_{f}\right\rangle \in \mathbf{Q}^{*}$ is the Petersson norm of a (suitably normalised) Jacquet-Langlands lift of $f$ to an eigenform $\phi_{f}$ on the definite quaternion algebra 
of discriminant $N^{-} \infty$ (cf. Sections 2.2 and 2.3 of [BD07]). Note that both $\mathbf{P}$ and $\ell$ depend on the choice of $K / \mathbf{Q}$, while the product $\ell \cdot \log _{A}^{2}(\mathbf{P})$ does not.

2.6. Ochiai's big dual exponential. We recall here the definition of Ochiai's two-variable big dual exponential for $\mathbb{T}$, constructed in [Och03] using previous work of Coleman-Perrin-Riou.

2.6.1. Notations. For every $n \in \mathbf{N} \cup\{\infty\}$, let $\mathbf{Q}_{p, n}$ be as in the introduction. The Galois group of $\mathbf{Q}_{p, \infty} / \mathbf{Q}_{p}$ is naturally identified with $G_{\infty}=\operatorname{Gal}\left(\mathbf{Q}_{\infty} / \mathbf{Q}\right)$, via the unique prime of $\mathbf{Q}_{\infty}$ dividing $p$.

Given $n \in \mathbf{N}$ and a $p$-adic representation $V$ of $G_{p}=G_{\mathbf{Q}_{p}}$, let $D_{\mathrm{dR}, n}(V):=H^{0}\left(\mathbf{Q}_{p, n}, V \otimes_{\mathbf{Q}_{p}} B_{\mathrm{dR}}\right)$, where $B_{\mathrm{dR}}$ is Fontaine's field of periods. It is equipped with a complete and separated decreasing filtration Fil $D_{\mathrm{dR}, n}(V)$, arising from the filtration $\left\{\mathrm{Fil}^{n} B_{\mathrm{dR}}:=t^{n} B_{\mathrm{dR}}^{+}\right\}_{n \in \mathbf{Z}}$, where $B_{\mathrm{dR}}^{+}$is the ring of integers of $B_{\mathrm{dR}}$ and $t:=\log \left(\zeta_{\infty}\right)$, for a fixed generator $\zeta_{\infty} \in \mathbf{Z}_{p}(1)$. Denote by $\operatorname{tg}_{n}(V):=D_{\mathrm{dR}, n}(V) / \mathrm{Fil}^{0}$ the tangent space of the $G_{\mathbf{Q}_{p, n}}$-representation $V$. If $n=0$, it will be omitted from the notations (e.g. $D_{\mathrm{dR}}(V)=D_{\mathrm{dR}, 0}(V)$ ). If $V$ is a de Rham representation of $G_{p}$, there is a natural $\operatorname{Gal}\left(\mathbf{Q}_{p, n} / \mathbf{Q}_{p}\right)$-equivariant isomorphism of filtered modules $D_{\mathrm{dR}, n}(V)=D_{\mathrm{dR}}(V) \otimes_{\mathbf{Q}_{p}} \mathbf{Q}_{p, n}$.

Let $S$ be a complete, local Noetherian ring with finite residue field of characteristic $p$ and let $\mathbb{X}$ be a free $S$-module of finite rank, equipped with a continuous $S$-linear action of $G_{p}$. Define

$$
H_{\mathrm{Iw}}^{q}\left(\mathbf{Q}_{p, \infty}, \mathbb{X}\right):=\lim _{n \in \mathbf{N}} H^{q}\left(\mathbf{Q}_{p, n}, \mathbb{X}\right),
$$

where the limit is taken with respect to the corestriction maps in Galois cohomology. Galois conjugation equips $H_{\mathrm{Iw}}^{q}\left(\mathbf{Q}_{p, \infty}, \mathbb{X}\right)$ with the structure of a module over the completed group algebra $\bar{S}:=S \llbracket G_{\infty} \rrbracket$.

For every $R$-module $\mathbb{M}$ and every $\nu \in \mathcal{X}^{\text {arith }}(R)$, write $\mathbb{M}_{\nu}:=\mathbb{M} \otimes_{R, \nu} \nu(R)$.

2.6.2. de Rham modules. Set $\check{\mathbb{T}}:=\operatorname{Hom}_{R}(\mathbb{T}, R)$ and $\check{\mathbb{T}}^{ \pm}:=\operatorname{Hom}_{R}\left(\mathbb{T}^{\mp}, R\right)$. Let $\nu \in \mathcal{X}^{\text {arith }}(R)$. Since $\mathbb{T}_{\nu}$ is a Galois-stable lattice in $V_{\nu}$ by $(12), \check{\mathbb{T}}_{\nu}$ is a Galois-stable lattice in the Deligne representation $\check{V}_{\nu}=\operatorname{Hom}_{K_{\nu}}\left(V_{\nu}, K_{\nu}\right)$ of $f_{\nu}$, where $K_{\nu}:=\operatorname{Frac}(\nu(R))$. Define $V_{\nu}^{ \pm}:=\mathbb{T}_{\nu}^{ \pm} \otimes \mathbf{z}_{p} \mathbf{Q}_{p}$ and $\check{V}_{\nu}^{ \pm}:=\check{\mathbb{T}}_{\nu}^{ \pm} \otimes \mathbf{z}_{p} \mathbf{Q}_{p}$. According to (10), for $M_{\nu} \in\left\{V_{\nu}, \check{V}_{\nu}\right\}$ there is a short exact sequence of $K_{\nu}\left[G_{p}\right]$-modules

$$
0 \longrightarrow M_{\nu}^{+} \stackrel{i^{+}}{\longrightarrow} M_{\nu} \stackrel{p^{-}}{\longrightarrow} M_{\nu}^{-} \longrightarrow 0 \text {. }
$$

The representation $\check{V}_{\nu}$ is known to be de Rham, and then so is $V_{\nu}$. In addition, Fil ${ }^{0} D_{\mathrm{dR}}\left(\check{V}_{\nu}\right)=D_{\mathrm{dR}}\left(\check{V}_{\nu}\right)$ and $\mathrm{Fil}^{m} D_{\mathrm{dR}}\left(\breve{V}_{\nu}\right)$ is 1 -dimensional over $K_{\nu}$ (resp., zero) for every $1 \leq m \leq k_{\nu}-1$ (resp., $m \geq k_{\nu}$ ), where $k_{\nu} \geq 2$ is the weight of $\nu$. It follows easily from (11) that $p^{-}: V_{\nu} \rightarrow V_{\nu}^{-}$and $i^{+}: \check{V}_{\nu}^{+} \hookrightarrow \check{V}_{\nu}$ induce isomorphisms of $K_{\nu}$-modules

$$
\mathrm{Fil}^{0} D_{\mathrm{dR}, n}\left(V_{\nu}\right) \cong D_{\mathrm{dR}, n}\left(V_{\nu}^{-}\right) ; \quad D_{\mathrm{dR}, n}\left(\check{V}_{\nu}^{+}(1)\right) \cong \operatorname{tg}_{n}\left(\check{V}_{\nu}(1)\right)
$$

for every $n \in \mathbf{N}$, which we consider as equalities in what follows.

For every $n \in \mathbf{N}$ the duality $V_{\nu} \times \check{V}_{\nu}(1) \rightarrow K_{\nu}(1)$ induces a $K_{\nu}$-bilinear form

$$
\langle-,-\rangle_{\mathrm{dR}}=\langle-,-\rangle_{\mathrm{dR}, n}: \mathrm{Fil}^{0} D_{\mathrm{dR}, n}\left(V_{\nu}\right) \times \operatorname{tg}_{n}\left(\check{V}_{\nu}(1)\right) \stackrel{\cup}{\longrightarrow} D_{\mathrm{dR}, n}\left(K_{\nu}(1)\right)=\mathbf{Q}_{p, n} \otimes \mathbf{Q}_{p} K_{\nu} .
$$

Under the isomorphisms $D_{\mathrm{dR}, n}(M)=D_{\mathrm{dR}}(M) \otimes_{\mathbf{Q}_{p}} \mathbf{Q}_{p, n}$, for $M=V_{\nu}, \check{V}_{\nu}(1)$, the pairing $\langle-,-\rangle_{\mathrm{dR}, n}$ is identified with the $\mathbf{Q}_{p, n}$-base change of $\langle-,-\rangle_{\mathrm{dR}, 0}$. Denote also by $\langle-,-\rangle_{\mathrm{dR}}: \mathrm{Fil}^{0} D_{\mathrm{dR}, n}\left(V_{\nu}\right) \times \operatorname{tg}_{n}\left(\check{V}_{\nu}(1)\right) \rightarrow K_{\nu}\left(\mu_{p^{n+1}}\right)$ the bilinear form defined by composing $\langle-,-\rangle_{\mathrm{dR}}$ with the multiplication $K_{\nu} \otimes_{\mathbf{Q}_{p}} \mathbf{Q}_{p, n} \rightarrow K_{\nu}\left(\mu_{p^{n+1}}\right)$.

2.6.3. Variation of periods. Let $\mathbf{Q}_{p}^{\mathrm{un}}$ be the maximal unramified extension of $\mathbf{Q}_{p}$ and let $\widehat{\mathbf{Z}}_{p}^{\text {un }}$ be the $p$-adic completion of its ring of integers. Following [Och03, Section 3], define the $R$-module

$$
\mathcal{D}:=H^{0}\left(\mathbf{Q}_{p}, \widehat{\mathbf{Z}}_{p}^{\mathrm{un}} \widehat{\otimes}_{\mathbf{z}_{p}} \check{\mathbb{T}}^{+}\right) \text {. }
$$

By (10) and (11), the $G_{p}$-module $\check{\mathbb{T}}^{+}$is unramified and free of rank one as an $R$-module. Then $\mathcal{D}$ is also a free $R$-module of rank one, by Lemma 3.3 of [Och03]. As $H^{0}\left(\mathbf{Q}_{p}^{\mathrm{un}}, B_{\mathrm{dR}}\right)=\widehat{\mathbf{Z}}_{p}^{\mathrm{un}} \otimes_{\mathbf{z}_{p}} \mathbf{Q}_{p}$, this easily implies (cf. loc. cit.) that for every $\nu \in \mathcal{X}^{\operatorname{arith}}(R)$ there is a natural isomorphism of $K_{\nu}$-modules $\mathcal{D}_{\nu} \otimes \mathbf{z}_{p} \mathbf{Q}_{p} \cong D_{\mathrm{dR}}\left(\check{V}_{\nu}^{+}\right)$. This induces a natural $\nu$-specialisation map

$$
\mathcal{D} \longrightarrow D_{\mathrm{dR}}\left(\check{V}_{\nu}^{+}\right)
$$

For every $X \in \mathcal{D}$, denote by $X_{\nu}$ the $\nu$-specialisation of $X$.

Fix a generator $\mho$ of the $R$-module $\mathcal{D}$, which also fixes a $K_{\nu}$-basis

$$
\mho_{\nu}(1):=\mho_{\nu} \otimes \zeta_{\mathrm{dR}} \in \operatorname{tg}\left(\check{V}_{\nu}(1)\right) .
$$

Here $\zeta_{\mathrm{dR}}:=\zeta_{\infty} \otimes \log \left(\zeta_{\infty}\right)^{-1} \in D_{\mathrm{dR}}\left(\mathbf{Q}_{p}(1)\right)$ is the canonical $\mathbf{Q}_{p}$-basis associated to a generator $\zeta_{\infty} \in \mathbf{Z}_{p}(1)$ and $\cdot \otimes \zeta_{\mathrm{dR}}$ is the natural isomorphism $D_{\mathrm{dR}}\left(\check{V}_{\nu}^{+}\right) \cong D_{\mathrm{dR}}\left(\check{V}_{\nu}^{+}\right) \otimes_{\mathbf{Q}_{p}} D_{\mathrm{dR}}\left(\mathbf{Q}_{p}(1)\right)=D_{\mathrm{dR}}\left(\check{V}_{\nu}^{+}(1)\right)$.

By (13) and (15) one has $\mathbb{T}_{\psi} \cong T_{p}(A)$ and $\mathbb{T}_{\psi}^{-} \cong \mathbf{Z}_{p}$ respectively. Then $\check{V}_{\psi}(1)$ and $\check{V}_{\psi}^{+}(1)$ are identified with $\check{V}_{p}(A)(1)$ and $\mathbf{Q}_{p}(1)$ respectively, where $\check{V}_{p}(A):=\operatorname{Hom}_{\mathbf{Q}_{p}}\left(V_{p}(A), \mathbf{Q}_{p}\right)$. In particular $\zeta_{\mathrm{dR}}$ can be identified with an element of $\operatorname{tg}\left(\check{V}_{p}(A)(1)\right)$ (cf. equation (24)). After possibly multiplying $\mho$ by a unit in $R$, we can assume

$$
\mho_{\psi}(1)=\zeta_{\mathrm{dR}} \in \operatorname{tg}\left(\check{V}_{p}(A)(1)\right) .
$$


2.6.4. Ochiai's two-variable big dual exponential. For every $\nu \in \mathcal{X}^{\text {arith }}(R)$ and every finite order character $\chi: G_{\infty} \rightarrow \overline{\mathbf{Q}}_{p}^{*}$ write $\nu \times \chi: \bar{R} \rightarrow \overline{\mathbf{Q}}_{p}$ for the unique morphism of $\mathbf{Z}_{p}$-algebras whose restriction to $R$ (resp., $G_{\infty}$ )

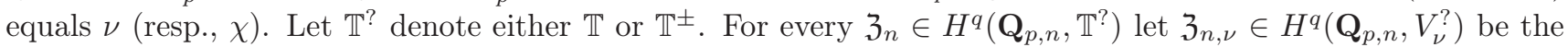
image of $\mathfrak{Z}_{n}$ under the morphism induced in cohomology by $\mathbb{T}^{?} \rightarrow \mathbb{T}_{\nu}^{\text {? }} \subset V_{\nu}^{?}$. Finally, for every $n \in \mathbf{N}$, write

$$
\exp ^{*}=\exp _{V_{\nu}^{-}}^{*}: H^{1}\left(\mathbf{Q}_{p, n}, V_{\nu}^{-}\right) \longrightarrow D_{\mathrm{dR}, n}\left(V_{\nu}^{-}\right) \cong \operatorname{Fil}^{0} D_{\mathrm{dR}, n}\left(V_{\nu}\right)
$$

for the Bloch-Kato dual exponential map defined in [Kat93, Chapter II].

The following proposition is proved in Section 5 of [Och03] (see in particular Proposition 5.1) building on previous work of Coleman [Col79] and Perrin-Riou [PR94].

Proposition 2.3. There exists a unique morphism of $\bar{R}$-modules

$$
\mathcal{L}_{\mathbb{T}}:=\mathcal{L}_{\mathbb{T}, \mho}: H_{\mathrm{Iw}}^{1}\left(\mathbf{Q}_{p, \infty}, \mathbb{T}^{-}\right) \longrightarrow \overline{\mathfrak{p}} \subset \bar{R}
$$

such that: for every $\mathfrak{Z}=\left(\mathfrak{Z}_{n}\right) \in H_{\mathrm{Iw}}^{1}\left(\mathbf{Q}_{p, \infty}, \mathbb{T}^{-}\right)$, every weight-two arithmetic point $\nu \in \mathcal{X}^{\text {arith }}(R)$ and every character $\chi: \operatorname{Gal}\left(\mathbf{Q}_{p, n} / \mathbf{Q}_{p}\right) \rightarrow \overline{\mathbf{Q}}_{p}^{*}$ of conductor $p^{m} \leq p^{n+1}$

$$
\nu \times \chi\left(\mathcal{L}_{\mathbb{T}}(\mathfrak{Z})\right)=\mathcal{E}(\nu, \chi) \sum_{\sigma \in \operatorname{Gal}\left(\mathbf{Q}_{p, n} / \mathbf{Q}_{p}\right)} \chi(\sigma)^{-1} \cdot\left\langle\exp ^{*}\left(\mathfrak{Z}_{n, \nu}^{\sigma}\right), \mho_{\nu}(1)\right\rangle_{\mathrm{dR}},
$$

where

$$
\mathcal{E}(\nu, \chi):=\tau(\chi) \nu\left(\mathbf{a}_{p}\right)^{-m}\left(1-\frac{\chi(p) \nu\left(\mathbf{a}_{p}\right)}{p}\right)^{-1}\left(1-\frac{\chi(p)}{\nu\left(\mathbf{a}_{p}\right)}\right) .
$$

With a slight abuse of notation, write again

$$
\mathcal{L}_{\mathbb{T}}: H_{\mathrm{Iw}}^{1}\left(\mathbf{Q}_{p, \infty}, \mathbb{T}\right) \longrightarrow \overline{\mathfrak{p}}
$$

for the composition of $\mathcal{L}_{\mathbb{T}}$ with the morphism $H_{\mathrm{Iw}}^{1}\left(\mathbf{Q}_{p, \infty}, \mathbb{T}\right) \rightarrow H_{\mathrm{Iw}}^{1}\left(\mathbf{Q}_{p, \infty}, \mathbb{T}^{-}\right)$induced by $p^{-}: \mathbb{T} \rightarrow \mathbb{T}^{-}$.

2.7. Beilinson-Kato elements and Kato's reciprocity law. We now state a general version of Kato's reciprocity law, following Section 6 of [Och06] (see in particular Corollary 6.17).

Denote by $\overline{\mathbf{Q}}(N p) / \mathbf{Q}$ the maximal algebraic extension of $\mathbf{Q}$ which is unramified at every finite prime $l \nmid N p$, and set $\mathfrak{G}_{n}:=\operatorname{Gal}\left(\overline{\mathbf{Q}}(N p) / \mathbf{Q}_{n}\right)$. Let $S$ be a local complete Noetherian ring with finite residue field of characteristic $p$ and let $\mathbb{X}$ be a free $S$-module of finite rank, equipped with a continuous $S$-linear action of $\mathfrak{G}_{0}$. Define

$$
H_{\mathrm{Iw}}^{q}\left(\mathbf{Q}_{\infty}, \mathbb{X}\right):=\lim _{n \in \mathbf{N}} H^{q}\left(\mathfrak{G}_{n}, \mathbb{X}\right)
$$

where the limit is taken with respect to the corestriction maps. According to [Rub00, Corollary B.3.6], if $q=1$ and $S=\mathbf{Z}_{p}$, the $\Lambda_{\text {cyc }}$-module $H_{\mathrm{Iw}}^{1}\left(\mathbf{Q}_{\infty}, \mathbb{X}\right)$ is isomorphic to the inverse limit of the cohomology groups $H^{1}\left(\mathbf{Q}_{n}, \mathbb{X}\right)$. In particular the definition of $H_{\mathrm{Iw}}^{1}\left(\mathbf{Q}_{\infty}, T_{p}(A)\right)$ given here agrees with the one given in the introduction.

TheOREM 2.4. There exists $\mathfrak{Z}_{\infty}^{\mathrm{BK}}=\left(\mathfrak{Z}_{n}^{\mathrm{BK}}\right)_{n \in \mathbf{N}} \in H_{\mathrm{Iw}}^{1}\left(\mathbf{Q}_{\infty}, \mathbb{T}\right)$ such that

$$
\mathcal{L}_{\mathbb{T}}\left(\operatorname{res}_{p}\left(\mathfrak{Z}_{\infty}^{\mathrm{BK}}\right)\right)=L_{p}(\mathbf{f})
$$

REMARK 2.5. The preceding theorem comes principally from the work of Kato [Kat04]. For every arithmetic point $\nu \in \mathcal{X}^{\operatorname{arith}}(R)$, [Kat04] attaches to $f_{\nu}$ a cyclotomic Euler system for $\mathbb{T}_{\nu}$, using Beilinson-Kato elements in the $K_{2}$ of modular curves. In particular this gives a class $\zeta_{\infty, \nu}^{\mathrm{BK}} \in H_{\mathrm{Iw}}^{1}\left(\mathbf{Q}_{\infty}, \mathbb{T}_{\nu}\right)$, related to the $p$-adic $L$ function $L_{p}\left(f_{\nu}\right)$ via the Perrin-Riou big dual exponential (see in particular Theorem 16.6 of [Kat04]). According to Theorem 6.11 of $[\mathbf{O c h 0 6}]$, the classes $\left\{\zeta_{\infty, \nu}^{\mathrm{BK}}\right\}_{\nu}$ can be interpolated by a two-variable Beilinson-Kato class $\mathfrak{Z}_{\infty}^{\mathrm{BK}} \in H_{\mathrm{Iw}}^{1}\left(\mathbf{Q}_{\infty}, \mathbb{T}\right)$, satisfying the conclusions of the theorem.

\section{The derivative of Ochiai's big dual exponential}

Consider the morphism of $\bar{R}$-modules

$$
\mathcal{L}_{\mathbb{T}}(\cdot, k, s):=\overline{\mathrm{M}} \circ \mathcal{L}_{\mathbb{T}}: H_{\mathrm{Iw}}^{1}\left(\mathbf{Q}_{p, \infty}, \mathbb{T}^{-}\right) \longrightarrow \mathscr{J} \subset \mathscr{A},
$$

defined as the composition of Ochiai's big dual exponential $\mathcal{L}_{\mathbb{T}}$ with the Mellin transform $\overline{\mathrm{M}}$; note that $\mathcal{L}_{\mathbb{T}}(\cdot, k, s)$ takes values in $\mathscr{J} \subset \mathscr{A}$ since $\overline{\mathrm{M}}$ maps by construction the ideal $\overline{\mathfrak{p}}$ into $\mathscr{J}$. With a slight abuse of notation, denote again by $\mathcal{L}_{\mathbb{T}}(\cdot, k, s): H_{\mathrm{Iw}}^{1}\left(\mathbf{Q}_{p, \infty}, \mathbb{T}\right) \longrightarrow \mathscr{J}$ the composition of $\mathcal{L}_{\mathbb{T}}(\cdot, k, s)$ with the morphism induced by the projection $p^{-}: \mathbb{T} \rightarrow \mathbb{T}^{-}$. The aim of this section is to prove Theorem 3.1 below, which gives a simple expression for the derivative of $\mathcal{L}_{\mathbb{T}}(\cdot, k, s)$. 
Denote by $\operatorname{rec}_{p}: \mathbf{Q}_{p}^{*} \rightarrow G_{p}^{\mathrm{ab}}:=G_{\mathbf{Q}_{p}}^{\mathrm{ab}}$ the local reciprocity map, normalised so that $\operatorname{rec}_{p}\left(p^{-1}\right)$ is an arithmetic Frobenius. It induces an isomorphism $\operatorname{rec}_{p}: \mathbf{Q}_{p}^{*} \widehat{\otimes} \mathbf{Q}_{p} \cong G_{p}^{\mathrm{ab}} \widehat{\otimes} \mathbf{Q}_{p}$, where $G \widehat{\otimes} \mathbf{Q}_{p}:=\left(\lim _{n \in \mathbf{N}} G / p^{n} G\right) \otimes \mathbf{z}_{p} \mathbf{Q}_{p}$ for every abelian group $G$. This yields an isomorphism of $\mathbf{Q}_{p}$-vector spaces

$$
H^{1}\left(\mathbf{Q}_{p}, \mathbf{Q}_{p}\right)=\operatorname{Hom}_{\text {cont }}\left(G_{p}^{\mathrm{ab}} \widehat{\otimes} \mathbf{Q}_{p}, \mathbf{Q}_{p}\right) \cong \operatorname{Hom}_{\text {cont }}\left(\mathbf{Q}_{p}^{*} \widehat{\otimes} \mathbf{Q}_{p}, \mathbf{Q}_{p}\right)=\operatorname{Hom}_{\text {cont }}\left(\mathbf{Q}_{p}^{*}, \mathbf{Q}_{p}\right),
$$

which we consider as an equality. For every $\mathfrak{Z}=\left(\mathfrak{Z}_{n}\right) \in H_{\mathrm{Iw}}^{1}\left(\mathbf{Q}_{p, \infty}, \mathbb{T}^{-}\right)$, the class $\mathfrak{Z}_{0, \psi} \in H^{1}\left(\mathbf{Q}_{p}, \mathbf{Q}_{p}\right)$ is then identified with a continuous morphism on $\mathbf{Q}_{p}^{*}$ (see Section 2.6.4 for the notations). Let

$$
\exp _{A}^{*}: H^{1}\left(\mathbf{Q}_{p}, V_{p}(A)\right) \rightarrow \operatorname{Fil}^{0} D_{\mathrm{dR}}\left(V_{p}(A)\right) \cong \mathbf{Q}_{p}
$$

be the Bloch-Kato dual exponential map (cf. (24)). Finally, set $e(1):=(1+p) \widehat{\otimes} \log _{p}(1+p)^{-1} \in \mathbf{Z}_{p}^{*} \widehat{\otimes} \mathbf{Q}_{p}$.

Theorem 3.1. 1. Let $\mathfrak{Z}=\left(\mathfrak{Z}_{n}\right) \in H_{\mathrm{Iw}}^{1}\left(\mathbf{Q}_{p, \infty}, \mathbb{T}^{-}\right)$and let $\mathfrak{z}:=\mathfrak{Z}_{0, \psi} \in \operatorname{Hom}_{\text {cont }}\left(\mathbf{Q}_{p}^{*}, \mathbf{Q}_{p}\right)$. Then

$$
\left(1-\frac{1}{p}\right) \mathcal{L}_{\mathbb{T}}(\mathfrak{Z}, k, s) \equiv \mathfrak{z}\left(p^{-1}\right) \cdot(s-1)-\frac{1}{2} \mathscr{L}_{p}(A) \cdot \mathfrak{z}(e(1)) \cdot(k-2) \quad\left(\bmod \mathscr{J}^{2}\right) .
$$

2. Let $\mathfrak{Z}=\left(\mathfrak{Z}_{n}\right) \in H_{\mathrm{Iw}}^{1}\left(\mathbf{Q}_{p, \infty}, \mathbb{T}\right)$ and let $\mathfrak{z}:=\mathfrak{Z}_{0, \psi} \in H^{1}\left(\mathbf{Q}_{p}, V_{p}(A)\right)$. Then

$$
\left(1-\frac{1}{p}\right) \mathcal{L}_{\mathbb{T}}(\mathfrak{Z}, k, s) \equiv \mathscr{L}_{p}(A) \cdot \exp _{A}^{*}(\mathfrak{z}) \cdot(s-k / 2)\left(\bmod \mathscr{J}^{2}\right) .
$$

The proof of Theorem 3.1 is given in Section 3.3. We consider separately the partial derivatives of $\mathcal{L}_{\mathbb{T}}(\cdot, k, s)$ with respect to the cyclotomic variable $s$ and the weight variable $k$. In order to compute the derivative in the cyclotomic direction, we make use of the work of Wiles [Wil78] and Coleman [Col79]. To compute the derivative in the weight direction, we prove the existence of an improved big dual exponential, and then invoke a formula

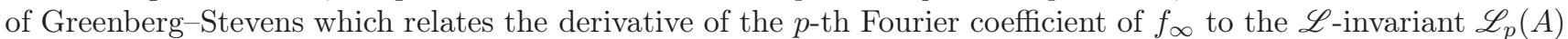
[GS93].

3.1. The Coleman map. In this section we first recall, following [Rub98], the definition of the cyclotomic big dual exponential $\mathcal{L}_{A}:=\mathcal{L}_{T_{p}(A)}$ for the $p$-adic Tate module of $A$, called the Coleman map. In our exceptional zero situation, it is a morphism of $\Lambda_{\text {cyc-algebras }}$

$$
\mathcal{L}_{A}: H_{\mathrm{Iw}}^{1}\left(\mathbf{Q}_{p, \infty}, T_{p}(A)\right) \longrightarrow I
$$

factoring through the Iwasawa cohomology of $\mathbf{Z}_{p}=T_{p}(A)^{-}$, where $I=I_{\text {cyc }}$ is the augmentation ideal of $\Lambda_{\text {cyc. }}$. We then prove in Proposition 3.6 a simple formula for its derivative at the augmentation ideal. While versions of Proposition 3.6 already appear in the literature (e.g. it follows from Proposition A.3.1 of [LVZ15]), we give here a proof in our setting for the convenience of the reader.

3.1.1. Definition of $\mathcal{L}_{A}$. For every $n \in \mathbf{N} \cup\{\infty\}$, identify $G_{n}:=\operatorname{Gal}\left(\mathbf{Q}_{n} / \mathbf{Q}\right)$ with the Galois group of $\mathbf{Q}_{p, n} / \mathbf{Q}_{p}$ via the unique prime of $\mathbf{Q}_{\infty}$ dividing $p$. Then $\Lambda_{\text {cyc }}=\mathbf{Z}_{p} \llbracket G_{\infty} \rrbracket$ is identified with the Iwasawa algebra of the cyclotomic $\mathbf{Z}_{p}$-extension $\mathbf{Q}_{p, \infty} / \mathbf{Q}_{p}$. Let $\mathbf{Z}_{p, n}$ and $\mathfrak{m}_{n}$ be the ring of integers of $\mathbf{Q}_{p, n}$ and its maximal ideal respectively, and let $N_{m, n}: \mathbf{Q}_{p, m}^{*} \rightarrow \mathbf{Q}_{p, n}^{*}$ be the norm map, for $m \geq n$.

Fix a generator $\zeta_{\infty}=\left(\zeta_{p^{n}}\right)_{n \in \mathbf{N}} \in \mathbf{Z}_{p}(1)$. As in [Rub98, Appendix], define for every $n \in \mathbf{N}$ :

$$
x_{n}:=p+\operatorname{Trace}_{\mathbf{Q}_{p}\left(\mu_{p^{n+1}}\right) / \mathbf{Q}_{p, n}}\left(\sum_{k=0}^{n} \frac{\zeta_{p^{n+1-k}}-1}{p^{k}}\right) \in \mathbf{Q}_{p, n} .
$$

A simple computation shows that these elements are compatible with respect to the trace maps. The following key lemma is due to Coleman (cf. Theorem 24 of [Col79]).

LEMma 3.2. There exists a unique principal unit $g(X) \in 1+(p, X) \cdot \mathbf{Z}_{p} \llbracket X \rrbracket$ such that:

1. $\log _{p}(g(0))=p$;

2. $\mathfrak{C}_{n}:=g\left(\zeta_{p^{n+1}}-1\right) \in 1+\mathfrak{m}_{n}$ and $\log _{p}\left(\mathfrak{C}_{n}\right)=x_{n}$ for every $n \in \mathbf{N}$;

3. $N_{m, n}\left(\mathfrak{C}_{m}\right)=\mathfrak{C}_{n}$ for every $m \geq n \geq 0$.

Proof. See [Rub98, Appendix] or [Rub00, Appendix D].

Identify $H^{1}\left(\mathbf{Q}_{p, n}, \mathbf{Z}_{p}(1)\right)=\mathbf{Q}_{p, n}^{*} \widehat{\otimes} \mathbf{Z}_{p}$ by Kummer theory. The preceding lemma allows us to define

$$
\mathfrak{C}:=\left(\mathfrak{C}_{n} \widehat{\otimes} 1\right)_{n \in \mathbf{N}} \in H_{\mathrm{Iw}}^{1}\left(\mathbf{Q}_{p, \infty}, \mathbf{Z}_{p}(1)\right) .
$$

Fix a topological generator $\sigma_{0} \in G_{\infty}$, and write $\varsigma:=\sigma_{0}-1 \in I$ for the corresponding generator of $I \subset \Lambda_{\text {cyc }}$.

Lemma 3.3. There exists a unique $\mathfrak{C}^{\prime}:=\mathfrak{C}_{\varsigma}^{\prime} \in H_{\mathrm{IW}}^{1}\left(\mathbf{Q}_{p, \infty}, \mathbf{Z}_{p}(1)\right)$ such that $\mathfrak{C}=\varsigma \cdot \mathfrak{C}^{\prime}$. 
Proof. The corestriction map induces an injective map: $H_{\mathrm{Iw}}^{1}\left(\mathbf{Q}_{p, \infty}, \mathbf{Z}_{p}(1)\right) / \varsigma \hookrightarrow H^{1}\left(\mathbf{Q}_{p}, \mathbf{Z}_{p}(1)\right)$, and the §-torsion submodule $H_{\mathrm{Iw}}^{1}\left(\mathbf{Q}_{\infty, p}, \mathbf{Z}_{p}(1)\right)[\varsigma]$ is trivial (being a quotient of $H^{0}\left(\mathbf{Q}_{p}, \mathbf{Z}_{p}(1)\right)$ ). It is then sufficient to prove that the principal unit $\mathfrak{C}_{0}$ is equal to 1 . Note that $x_{0}=0$, as $\operatorname{Trace}_{\mathbf{Q}_{p}\left(\mu_{p}\right) / \mathbf{Q}_{p}}\left(\zeta_{p}-1\right)=-p$. By Lemma $3.2(2)$ this implies $\log _{p}\left(\mathfrak{C}_{0}\right)=0$, i.e. $\mathfrak{C}_{0}=1($ as $p \neq 2)$.

By local Tate duality, there is a natural morphism of $\Lambda_{\text {cyc-modules }}$

$$
\langle-,-\rangle_{\infty}: H_{\mathrm{Iw}}^{1}\left(\mathbf{Q}_{p, \infty}, \mathbf{Z}_{p}\right) \otimes_{\Lambda_{\mathrm{cyc}}} H_{\mathrm{Iw}}^{1}\left(\mathbf{Q}_{p, \infty}, \mathbf{Z}_{p}(1)\right)^{\iota} \longrightarrow \Lambda_{\mathrm{cyc}}
$$

Here $\iota$ is Iwasawa's main involution on $\Lambda_{\text {cyc }}$, i.e. the isomorphism of $\mathbf{Z}_{p}$-algebras which acts as inversion on $G_{\infty}$, and $H_{\mathrm{Iw}}^{1}\left(\mathbf{Q}_{p, \infty}, \mathbf{Z}_{p}(1)\right)^{\iota}$ denotes the $\mathbf{Z}_{p}$-module $H_{\mathrm{Iw}}^{1}\left(\mathbf{Q}_{p, \infty}, \mathbf{Z}_{p}(1)\right)$, with $\Lambda_{\text {cyc-action obtained by twisting the }}$ original action by $\iota$. (See, e.g. Section 2.1.5 of [PR92] for the definition of $\langle-,-\rangle_{\infty}$.) Define

$$
\mathcal{L}_{A}:=-\langle\cdot, \mathfrak{C}\rangle_{\infty}: H_{\mathrm{Iw}}^{1}\left(\mathbf{Q}_{p, \infty}, \mathbf{Z}_{p}\right) \longrightarrow I
$$

The fact that $\mathcal{L}_{A}$ takes values in the augmentation ideal follows from Lemma 3.3 (as $\iota(\varsigma)=-\sigma_{0}^{-1} \varsigma \in I$ ). The following proposition is a version of the Coleman-Wiles explicit reciprocity law [Wil78], [Col79]; we refer to [Rub98, Appendix] (or [Ven13, Section 13.2]) for a proof in our setting.

Proposition 3.4. For every $z=\left(z_{n}\right) \in H_{\mathrm{Iw}}^{1}\left(\mathbf{Q}_{p, \infty}, \mathbf{Z}_{p}\right)$ and every non-trivial character $\chi$ of $G_{n}$

$$
\chi\left(\mathcal{L}_{A}(z)\right)=\tau(\chi) \sum_{\sigma \in G_{n}} \chi(\sigma)^{-1} \cdot \exp _{n}^{*}\left(z_{n}^{\sigma}\right),
$$

where $\exp _{n}^{*}: H^{1}\left(\mathbf{Q}_{p, n}, \mathbf{Q}_{p}\right) \rightarrow D_{\mathrm{dR}, n}\left(\mathbf{Q}_{p}\right)=\mathbf{Q}_{p, n}$ is the Bloch-Kato dual exponential map.

With a slight abuse of notation, denote again by $\mathcal{L}_{A}: H_{\mathrm{Iw}}^{1}\left(\mathbf{Q}_{p, \infty}, T_{p}(A)\right) \longrightarrow I$ the composition of $\mathcal{L}_{A}$ with the map induced by the projection $p^{-}: T_{p}(A) \rightarrow \mathbf{Z}_{p}$ (see (14)). Note the following corollary.

Corollary 3.5. Let $\mathbb{T}^{\text {? }}$ denote either $\mathbb{T}^{-}$or $\mathbb{T}$. For every $\mathfrak{Z} \in H_{\mathrm{Iw}}^{1}\left(\mathbf{Q}_{p, \infty}, \mathbb{T}^{?}\right)$ :

$$
\psi\left(\mathcal{L}_{\mathbb{T}}(\mathfrak{Z})\right)=\mathcal{L}_{A}\left(\mathfrak{Z}_{\psi}\right)
$$

where $\mathfrak{Z}_{\psi} \in H_{\mathrm{Iw}}^{1}\left(\mathbf{Q}_{p, \infty}, T_{p}(A)^{?}\right)$ is the image of $\mathfrak{Z}$ under the morphism induced by $\mathbb{T}^{?} \rightarrow \mathbb{T}_{\psi}^{?} \cong T_{p}(A)^{?}$.

Proof. As $\psi\left(\mathbf{a}_{p}\right)=1$, this follows from (25) and the interpolation properties of $\psi \circ \mathcal{L}_{\mathbb{T}}$ and $\mathcal{L}_{A}$.

3.1.2. The derivative of $\mathcal{L}_{A}$. If $M$ denotes either $T_{p}(A)$ or $\mathbf{Z}_{p}$, define the derivative of $\mathcal{L}_{A}$ :

$$
\mathcal{L}_{A}^{\prime}: H_{\mathrm{Iw}}^{1}\left(\mathbf{Q}_{p, \infty}, M\right) \longrightarrow I / I^{2}
$$

as the composition of $\mathcal{L}_{A}$ with the projection $\{\cdot\}: I \rightarrow I / I^{2}$. Set $\log _{p}(\varsigma):=\log _{p}\left(\chi_{\text {cyc }}\left(\sigma_{0}\right)\right)$ and

$$
l_{\varsigma}:=\log _{p}(\varsigma) \cdot\left(1-p^{-1}\right) \in \mathbf{Z}_{p}^{*},
$$

where $\varsigma=\sigma_{0}-1$ is our fixed generator of $I$. As in Section 3, the cohomology group $H^{1}\left(\mathbf{Q}_{p}, \mathbf{Z}_{p}\right)$ is identified with $\mathrm{Hom}_{\text {cont }}\left(\mathbf{Q}_{p}^{*}, \mathbf{Z}_{p}\right)$ via the local reciprocity map.

Proposition 3.6. Let $z=\left(z_{n}\right) \in H_{\mathrm{IW}}^{1}\left(\mathbf{Q}_{p, \infty}, \mathbf{Z}_{p}\right)$. Then $l_{\varsigma} \cdot \mathcal{L}_{A}^{\prime}(z)=z_{0}\left(p^{-1}\right)\{\varsigma\}$.

Before giving the proof of Proposition 3.6, we deduce the following corollary.

Corollary 3.7. Let $z=\left(z_{n}\right) \in H_{\mathrm{Iw}}^{1}\left(\mathbf{Q}_{p, \infty}, T_{p}(A)\right)$. Then

$$
l_{\varsigma} \cdot \mathcal{L}_{A}^{\prime}(z)=\mathscr{L}_{p}(A) \cdot \exp _{A}^{*}\left(z_{0}\right)\{\varsigma\} .
$$

In particular $\mathcal{L}_{A}(z) \in I^{2}$ if and only if $z_{0} \in H_{f}^{1}\left(\mathbf{Q}_{p}, V_{p}(A)\right) \cong A\left(\mathbf{Q}_{p}\right) \widehat{\otimes} \mathbf{Q}_{p}$.

Proof. Consider the exact sequence

$$
H^{1}\left(\mathbf{Q}_{p}, \mathbf{Q}_{p}(1)\right) \stackrel{i^{+}}{\longrightarrow} H^{1}\left(\mathbf{Q}_{p}, V_{p}(A)\right) \stackrel{p^{-}}{\longrightarrow} \operatorname{Hom}_{\text {cont }}\left(\mathbf{Q}_{p}^{*}, \mathbf{Q}_{p}\right) \stackrel{\delta}{\longrightarrow} H^{2}\left(\mathbf{Q}_{p}, \mathbf{Q}_{p}(1)\right) \stackrel{\text { inv }_{p}}{\cong} \mathbf{Q}_{p}
$$

arising from the exact sequence (14), where $\operatorname{inv}_{p}$ is the invariant map of local class field theory. A direct computation shows that $\delta(\cdot)=\operatorname{inv}_{p}\left(\cdot \cup q_{A} \widehat{\otimes} 1\right)$, where $\cup: H^{1}\left(\mathbf{Q}_{p}, \mathbf{Q}_{p}\right) \times H^{1}\left(\mathbf{Q}_{p}, \mathbf{Q}_{p}(1)\right) \rightarrow H^{2}\left(\mathbf{Q}_{p}, \mathbf{Q}_{p}(1)\right)$ is the natural cup-product pairing and we identify as above $H^{1}\left(\mathbf{Q}_{p}, \mathbf{Q}_{p}(1)\right)=\mathbf{Q}_{p}^{*} \widehat{\otimes} \mathbf{Q}_{p}$. It then follows by local class field theory [Ser67] that $\delta(\phi)=-\phi\left(q_{A}\right)$ for every $\phi \in \operatorname{Hom}_{\text {cont }}\left(\mathbf{Q}_{p}^{*}, \mathbf{Q}_{p}\right)$, so that the image of $p^{-}$is equal to the space of morphisms $\phi$ such that $\phi\left(q_{A}\right)=0$. As $\log _{p}$ and $\operatorname{ord}_{p}$ form a $\mathbf{Q}_{p}$-basis of $\operatorname{Hom}_{\text {cont }}\left(\mathbf{Q}_{p}^{*}, \mathbf{Q}_{p}\right)$, this implies

$$
\operatorname{Im}\left(p^{-}\right)=\mathbf{Q}_{p} \cdot \log _{q_{A}},
$$

where $\log _{q_{A}}=\log _{p}-\mathscr{L}_{p}(A) \cdot \operatorname{ord}_{p}$ is the branch of the $p$-adic logarithm which vanishes on $q_{A} \in p \mathbf{Z}_{p}$. 
Let $z=\left(z_{n}\right) \in H_{\mathrm{Iw}}^{1}\left(\mathbf{Q}_{p}, T_{p}(A)\right)$, and write $p^{-}\left(z_{0}\right)=\alpha \cdot \log _{q_{A}} \in \operatorname{Hom}_{\mathrm{cont}}\left(\mathbf{Q}_{p}^{*}, \mathbf{Q}_{p}\right)$, for some $\alpha \in \mathbf{Q}_{p}$. Then $\exp _{A}^{*}\left(z_{0}\right)=\exp ^{*}\left(\alpha \cdot \log _{q_{A}}\right)=\alpha$, where $\exp ^{*}=\exp _{0}^{*}$ is the Bloch-Kato dual exponential for $\mathbf{Q}_{p}$. Indeed, by its very definition (see Chapter II of [Kat93]), $\exp ^{*}\left(\log _{p}\right)=1$ and $\exp ^{*}\left(\operatorname{ord}_{p}\right)=0$. According Proposition 3.6

$$
l_{\varsigma} \cdot \mathcal{L}_{A}^{\prime}(z)=\alpha \log _{q_{A}}\left(p^{-1}\right) \cdot\{\varsigma\}=\mathscr{L}_{p}(A) \cdot \exp _{A}^{*}\left(z_{0}\right) \cdot\{\varsigma\}
$$

The last assertion in the statement follows from the non-vanishing of the $\mathscr{L}$-invariant [BSDGP96] and the fact that the finite part $H_{f}^{1}\left(\mathbf{Q}_{p}, V_{p}(A)\right) \cong A\left(\mathbf{Q}_{p}\right) \widehat{\otimes} \mathbf{Q}_{p}[\mathbf{B K 9 0}]$ of the local cohomology group $H^{1}\left(\mathbf{Q}_{p}, V_{p}(A)\right)$ is the kernel of the dual exponential. Indeed, the preceding discussion shows that an element of $H^{1}\left(\mathbf{Q}_{p}, V_{p}(A)\right)$ belongs to the kernel of $\exp _{A}^{*}$ if and only if it is in the image of $i^{+}: H^{1}\left(\mathbf{Q}_{p}, \mathbf{Q}_{p}(1)\right) \rightarrow H^{1}\left(\mathbf{Q}_{p}, V_{p}(A)\right)$, and the latter equals $H_{f}^{1}\left(\mathbf{Q}_{p}, V_{p}(A)\right)$, as follows easily from Kummer theory and the surjectivity of the Tate parametrisation (3).

Proof of Proposition 3.6. For every $n \in \mathbf{N}$, let $\pi_{n}:=\operatorname{Norm}_{\mathbf{Q}_{p}\left(\mu_{p^{n+1}}\right) / \mathbf{Q}_{p, n}}\left(\zeta_{p^{n+1}}-1\right)$; this is a uniformiser of $\mathbf{Z}_{p, n}$. Since $\mathbf{Q}_{p, n}^{*}$ has no non-trivial $p$-torsion, one has a decomposition

$$
H^{1}\left(\mathbf{Q}_{p, n}, \mathbf{Z}_{p}(1)\right)=\mathbf{Q}_{p, n}^{*} \widehat{\otimes} \mathbf{Z}_{p}=\widehat{\pi_{n}} \oplus 1+\mathfrak{m}_{n},
$$

where $\widehat{\pi_{n}}$ is the $p$-adic completion of $\pi_{n}^{\mathbf{Z}}$. Given $\alpha_{n} \in H^{1}\left(\mathbf{Q}_{p, n}, \mathbf{Z}_{p}(1)\right)$, let $\kappa_{n}\left(\alpha_{n}\right) \in 1+\mathfrak{m}_{n}$ be its projection to principal units, and $\operatorname{ord}_{n}\left(\alpha_{n}\right) \in \mathbf{Z}_{p}$ its $\pi_{n}$-adic valuation. Since $N_{m, n}\left(\pi_{m}\right)=\pi_{n}$ for every integers $m \geq n$, if $\alpha=\left(\alpha_{n}\right) \in H_{\mathrm{Iw}}^{1}\left(\mathbf{Q}_{p, \infty}, \mathbf{Z}_{p}(1)\right)$ then $\operatorname{ord}(\alpha):=\operatorname{ord}_{n}\left(\alpha_{n}\right)$ is independent of $n \in \mathbf{N}$, and $\kappa(\alpha):=\left(\kappa_{n}\left(\alpha_{n}\right)\right)_{n \in \mathbf{N}}$ is a compatible sequence with respect to the norm maps. One can then define maps

$$
\text { ord }: H_{\mathrm{Iw}}^{1}\left(\mathbf{Q}_{p, \infty}, \mathbf{Z}_{p}(1)\right) \rightarrow \mathbf{Z}_{p} ; \kappa: H_{\mathrm{Iw}}^{1}\left(\mathbf{Q}_{p, \infty}, \mathbf{Z}_{p}(1)\right) \rightarrow U_{\infty}^{1}
$$

where $U_{\infty}^{1}$ denotes the inverse limit of the groups $1+\mathfrak{m}_{n}$. Write $\pi_{\infty}:=\left(\pi_{n}\right) \in H_{\mathrm{Iw}}^{1}\left(\mathbf{Q}_{p, \infty}, \mathbf{Z}_{p}(1)\right)$. By construction $\alpha=\pi_{\infty}^{\text {ord }(\alpha)}+\kappa(\alpha)$ for every $\alpha=\left(\alpha_{n}\right) \in H_{\mathrm{IW}}^{1}\left(\mathbf{Q}_{p, \infty}, \mathbf{Z}_{p}(1)\right)$. Moreover, one has

$$
\alpha_{0}=p^{\operatorname{ord}(\alpha)} \in H^{1}\left(\mathbf{Q}_{p}, \mathbf{Z}_{p}(1)\right) \text {. }
$$

Indeed, local class field theory tells us that the image of the injective map $H_{\mathrm{Iw}}^{1}\left(\mathbf{Q}_{p, \infty}, \mathbf{Z}_{p}(1)\right) / \varsigma \hookrightarrow H^{1}\left(\mathbf{Q}_{p}, \mathbf{Z}_{p}(1)\right)$ induced by the corestriction equals $\widehat{p}=\widehat{\pi_{0}}$. Then $U_{\infty}^{1} \subset \varsigma \cdot H_{\mathrm{Iw}}^{1}\left(\mathbf{Q}_{p, \infty}, \mathbf{Z}_{p}(1)\right)$ and equation (26) follows.

Let us now consider the element $\mathfrak{C}^{\prime}=\mathfrak{C}_{\varsigma}^{\prime}$ appearing in Lemma 3.3. For every $z=\left(z_{n}\right) \in H_{\mathrm{Iw}}^{1}\left(\mathbf{Q}_{p, \infty}, \mathbf{Z}_{p}\right)$

$$
\mathcal{L}_{A}^{\prime}(z)=z_{0}\left(p^{-1}\right) \cdot \operatorname{ord}\left(\mathfrak{C}^{\prime}\right) \cdot\{\varsigma\}
$$

Indeed, let $\langle-,-\rangle: H^{1}\left(\mathbf{Q}_{p}, \mathbf{Z}_{p}\right) \times H^{1}\left(\mathbf{Q}_{p}, \mathbf{Z}_{p}(1)\right) \rightarrow \mathbf{Z}_{p}$ be the local Tate pairing. Then $\left\langle z_{0}, \mathfrak{C}_{0}^{\prime}\right\rangle=\varepsilon\left(\left\langle z, \mathfrak{C}^{\prime \prime}\right\rangle_{\infty}\right)$, where $\varepsilon$ is the augmentation map and we write $\mathfrak{C}^{\prime}=\left(\mathfrak{C}_{n}^{\prime}\right)$. This implies

$$
\mathcal{L}_{A}^{\prime}(z)=-\left\{\left\langle z, \varsigma \cdot \mathfrak{C}^{\prime \prime}\right\rangle_{\infty}\right\}=\left\langle z_{0}, \mathfrak{C}_{0}^{\prime}\right\rangle \cdot\{\varsigma\}
$$

(Note that $\iota(\varsigma) \equiv-\varsigma \bmod I^{2}$.) Since $\left\langle z_{0}, x\right\rangle=z_{0}\left(x^{-1}\right)$ for every $x \in \mathbf{Q}_{p}^{*} \widehat{\otimes} \mathbf{Z}_{p}$ by local class field theory [Ser67], equation (27) follows by combining equations (28) and (26).

Thanks to (27), the proposition will follow once we prove the claim

$$
\operatorname{ord}\left(\mathfrak{C}^{\prime}\right)=l_{\varsigma}^{-1} \in \mathbf{Z}_{p}^{*}
$$

Write $V_{\infty}$ for the inverse limit of the groups $\mathbf{Z}_{p}\left[\zeta_{p^{m+1}}\right]^{*}$, for $m \in \mathbf{N}$. According to Theorem A of [Col79], for every $v=\left(v_{n}\right) \in V_{\infty}$ there exists a unique power series $f_{v}(T) \in \mathbf{Z}_{p} \llbracket T \rrbracket^{*}$ such that $f_{v}\left(\zeta_{p^{n+1}}-1\right)=v_{n}$ for every $n \in \mathbf{N}$. The association $v \mapsto f_{v}(T)$ is a morphism of $\mathbf{Z}_{p} \llbracket \operatorname{Gal}\left(\mathbf{Q}_{p}\left(\mu_{p} \infty\right) / \mathbf{Q}_{p}\right) \rrbracket$-modules (see [Col79] for details). Note that, with the notations of Lemma $3.2, g(T)=f_{\mathfrak{C}}(T)$. As $\mathfrak{C}=\varsigma \cdot \mathfrak{C}^{\prime}$ and $\mathfrak{C}^{\prime}=\kappa\left(\mathfrak{C}^{\prime}\right)+\pi_{\infty}^{\text {ord }\left(\mathfrak{C}^{\prime}\right)}$, one then finds

$$
g(T)=\frac{f_{\kappa\left(\mathfrak{C}^{\prime}\right)}\left((1+T)^{\chi_{\mathrm{cyc}}\left(\sigma_{0}\right)}-1\right)}{f_{\kappa\left(\mathfrak{C}^{\prime}\right)}(T)} \cdot\left(\prod_{\mu \in \mu_{p-1}} \frac{(1+T)^{\mu \cdot \chi_{\mathrm{cyc}}\left(\sigma_{0}\right)}-1}{(1+T)^{\mu}-1}\right)^{\operatorname{ord}\left(\mathfrak{C}^{\prime \prime}\right)}
$$

Evaluating this equality at $T=0$ and then applying the $p$-adic logarithm, we easily obtain

$$
\log _{p}(g(0))=(p-1) \cdot \operatorname{ord}\left(\mathfrak{C}^{\prime}\right) \cdot \log _{p}\left(\chi_{\mathrm{cyc}}\left(\sigma_{0}\right)\right)=p \cdot \operatorname{ord}\left(\mathfrak{C}^{\prime \prime}\right) \cdot l_{\varsigma} .
$$

Since $\log _{p}(g(0))=p$ by Lemma $3.2(1)$, the claim (29) follows. 
3.2. The improved big dual exponential. The aim of this section is to construct an improved big dual exponential $\mathcal{L}_{\mathbb{T}}^{*}: H^{1}\left(\mathbf{Q}_{p}, \mathbb{T}^{-}\right) \rightarrow R[1 / p]$. To do this we follow the techniques of [Och03, Section 5].

Proposition 3.8. There exists a unique morphism of $R$-modules

$$
\mathcal{L}_{\mathbb{T}}^{*}=\mathcal{L}_{\mathbb{T}, \mho}^{*}: H^{1}\left(\mathbf{Q}_{p}, \mathbb{T}^{-}\right) \longrightarrow R \otimes \mathbf{z}_{p} \mathbf{Q}_{p}
$$

such that: for every $\mathfrak{Z} \in H^{1}\left(\mathbf{Q}_{p}, \mathbb{T}^{-}\right)$and every $\nu \in \mathcal{X}^{\operatorname{arith}}(R)$

$$
\nu\left(\mathcal{L}_{\mathbb{T}}^{*}(\mathfrak{Z})\right)=\left(1-\frac{\nu\left(\mathbf{a}_{p}\right)}{p}\right)^{-1}\left\langle\exp ^{*}\left(\mathfrak{Z}_{\nu}\right), \mho_{\nu}(1)\right\rangle_{\mathrm{dR}},
$$

where $\exp ^{*}: H^{1}\left(\mathbf{Q}_{p}, V_{\nu}^{-}\right) \rightarrow D_{\mathrm{dR}}\left(V_{\nu}^{-}\right)=\mathrm{Fil}^{0} D_{\mathrm{dR}}\left(V_{\nu}\right)$ is the Bloch-Kato dual exponential map.

Before giving the proof of Proposition 3.8, we note the following corollary (cf. Section 2.3.2).

Corollary 3.9. Let $\varepsilon: \bar{R} \rightarrow R$ be the augmentation map, and let $\mathfrak{Z}=\left(\mathfrak{Z}_{n}\right) \in H_{\mathrm{Iw}}^{1}\left(\mathbf{Q}_{p, \infty}, \mathbb{T}^{-}\right)$. Then

$$
\varepsilon\left(\mathcal{L}_{\mathbb{T}}(\mathfrak{Z})\right)=\left(1-\mathbf{a}_{p}^{-1}\right) \cdot \mathcal{L}_{\mathbb{T}}^{*}\left(\mathfrak{Z}_{0}\right) .
$$

Proof. Taking $\chi$ as the trivial character of $G_{\infty}$ in Proposition 2.3, one has

$$
\nu \circ \varepsilon\left(\mathcal{L}_{\mathbb{T}}(\mathfrak{Z})\right)=\left(1-\nu\left(\mathbf{a}_{p}\right)^{-1}\right) \cdot \nu\left(\mathcal{L}_{\mathbb{T}}^{*}\left(\mathfrak{Z}_{0}\right)\right),
$$

for every weight-two arithmetic point $\nu \in \mathcal{X}^{\operatorname{arith}}(R)$. Since such points (or better their kernels) form a dense subset of $\operatorname{Spec}(R)$, the corollary follows.

Proof of Proposition 3.8. Let $K$ be a complete subfield of $\widehat{\mathbf{Q}}_{p}^{\text {un }}$ and let $V$ be a $p$-adic representation of $G_{K}$. Denote by $D_{\mathrm{dR}, K}(V):=H^{0}\left(K, V \otimes_{\mathbf{Q}_{p}} B_{\mathrm{dR}}\right)$, and by exp : $D_{\mathrm{dR}, K}(V) \rightarrow H^{1}(K, V)$ the Bloch-Kato exponential map [BK90]. For $V=\mathbf{Q}_{p}(1)$, it is described by the composition

$$
\exp _{p}: D_{\mathrm{dR}, K}\left(\mathbf{Q}_{p}(1)\right)=K \longrightarrow K^{*} \widehat{\otimes} \mathbf{Q}_{p}=H^{1}\left(K, \mathbf{Q}_{p}(1)\right),
$$

where the first equality refers to the canonical identification $D_{\mathrm{dR}, K}\left(\mathbf{Q}_{p}(1)\right)=K \cdot \zeta_{\mathrm{dR}} \cong K$ (see Section 2.6.3), the arrow is given by the usual $p$-adic exponential and the last equality is the Kummer isomorphism. As $K$ is unramified, $\exp _{p}$ maps the ring of integers of $K$ into $\frac{1}{p} H^{1}\left(K, \mathbf{Z}_{p}(1)\right) \subset H^{1}\left(K, \mathbf{Q}_{p}(1)\right)$.

Set $G_{p}:=G_{\mathbf{Q}_{p}}, I_{p}:=I_{\mathbf{Q}_{p}}$ and $G_{p}^{\text {un }}:=G_{p} / I_{p}$. With the notations of Section 2.6, consider the morphism of $R\left[G_{p}^{\mathrm{un}}\right]$-modules

$$
\exp _{p} \widehat{\otimes} \text { id : } \widehat{\mathbf{Z}}_{p}^{\mathrm{un}} \widehat{\mathbf{z}}_{p} \check{\mathbb{T}}^{+} \rightarrow\left(H^{1}\left(I_{p}, \mathbf{Z}_{p}(1)\right) \widehat{\otimes}_{\mathbf{z}_{p}} \check{\mathbb{T}}^{+}\right) \otimes \mathbf{z}_{p} \mathbf{Q}_{p}=H^{1}\left(I_{p}, \check{\mathbb{T}}^{+}(1)\right) \otimes \mathbf{z}_{p} \mathbf{Q}_{p}
$$

(recall that $\check{\mathbb{T}}^{+}$is unramified). As $H^{0}\left(I_{p}, \check{\mathbb{T}}^{+}(1)\right)=0$, restriction gives an isomorphism between $H^{1}\left(\mathbf{Q}_{p}, \check{\mathbb{T}}^{+}(1)\right)$ and $H^{0}\left(G_{p}^{\text {un }}, H^{1}\left(I_{p}, \check{\mathbb{T}}^{+}(1)\right)\right)$. Taking $G_{p}^{\text {un }}$-invariants in (30) then yields a morphism of $R$-modules

$$
\exp _{\mathbb{T}}: \mathcal{D} \longrightarrow H^{1}\left(\mathbf{Q}_{p}, \check{\mathbb{T}}^{+}(1)\right) \otimes \mathbf{z}_{p} \mathbf{Q}_{p}
$$

We claim that for every arithmetic point $\nu \in \mathcal{X}^{\operatorname{arith}}(R)$

$$
\nu_{*}\left(\exp _{\mathbb{T}}(\mho)\right)=\exp \left(\mho_{\nu}(1)\right)
$$

where $\nu_{*}: H^{1}\left(\mathbf{Q}_{p}, \check{\mathbb{T}}^{+}(1)\right) \rightarrow H^{1}\left(\mathbf{Q}_{p}, \check{V}_{\nu}^{+}(1)\right)$ is the morphism induced by $\check{\mathbb{T}}^{+} \rightarrow \check{\mathbb{T}}_{\nu}^{+} \subset \check{V}_{\nu}^{+}$, and exp is the exponential on $D_{\mathrm{dR}}\left(\check{V}_{\nu}^{+}(1)\right)$. As above, the restriction map gives an isomorphism between $H^{1}\left(\mathbf{Q}_{p}, \check{V}_{\nu}^{+}(1)\right)$ and the $G_{p}^{\mathrm{un}}$-invariants of $H^{1}\left(I_{p}, \check{V}_{\nu}^{+}(1)\right)$. It follows that the exponential $\exp : D_{\mathrm{dR}}\left(\check{V}_{\nu}^{+}(1)\right) \rightarrow H^{1}\left(\mathbf{Q}_{p}, \check{V}_{\nu}^{+}(1)\right)$ is identified with the restriction of

$$
\exp _{p} \otimes \mathrm{id}: \widehat{\mathbf{Q}}_{p}^{\mathrm{un}} \otimes \mathbf{Q}_{p} \check{V}_{\nu}^{+} \longrightarrow H^{1}\left(I_{p}, \mathbf{Q}_{p}(1)\right) \otimes \mathbf{Q}_{p} \check{V}_{\nu}^{+}=H^{1}\left(I_{p}, \check{V}_{\nu}^{+}(1)\right)
$$

to the $G_{p}^{\text {un }}$-invariants. Equation (31) then follows from the definitions of $\exp _{\mathbb{T}}$ and $\mho_{\nu}(1)$.

Let $\langle-,-\rangle_{R}: H^{1}\left(\mathbf{Q}_{p}, \mathbb{T}^{-}\right) \otimes_{R} H^{1}\left(\mathbf{Q}_{p}, \check{\mathbb{T}}^{+}(1)\right) \rightarrow R$ be the $R$-adic local Tate pairing and define

$$
\exp _{\mathbb{T}}^{*}=\exp _{\mathbb{T}, \mho}^{*}:=\left\langle\cdot, \exp _{\mathbb{T}}(\mho)\right\rangle_{R}: H^{1}\left(\mathbf{Q}_{p}, \mathbb{T}^{-}\right) \longrightarrow R \otimes \mathbf{z}_{p} \mathbf{Q}_{p} .
$$

By (31) one obtains: for every $\mathfrak{Z} \in H^{1}\left(\mathbf{Q}_{p}, \mathbb{T}^{-}\right)$and every $\nu \in \mathcal{X}^{\operatorname{arith}}(R)$

$$
\nu\left(\exp _{\mathbb{T}}^{*}(\mathfrak{Z})\right)=\left\langle\mathfrak{Z}_{\nu}, \nu_{*}\left(\exp _{\mathbb{T}}(\mho)\right)\right\rangle_{\nu}=\left\langle\mathfrak{Z}_{\nu}, \exp \left(\mho_{\nu}(1)\right)\right\rangle_{\nu}=\left\langle\exp ^{*}\left(\mathfrak{Z}_{\nu}\right), \mho_{\nu}(1)\right\rangle_{\mathrm{dR}} .
$$

Here $\langle-,-\rangle_{\nu}: H^{1}\left(\mathbf{Q}_{p}, V_{\nu}^{-}\right) \times H^{1}\left(\mathbf{Q}_{p}, \check{V}_{\nu}^{+}(1)\right) \rightarrow K_{\nu}$ is the local Tate pairing and exp* is the Bloch-Kato dual exponential map on $H^{1}\left(\mathbf{Q}_{p}, V_{\nu}^{-}\right)$; the first equality follows from the functoriality of the local Tate duality, while the last equality is [Kat93, Chapter II, Theorem 1.4.1]. Define

$$
\mathcal{L}_{\mathbb{T}}^{*}:=\left(1-\frac{\mathbf{a}_{p}}{p}\right)^{-1} \exp _{\mathbb{T}}^{*}: H^{1}\left(\mathbf{Q}_{p}, \mathbb{T}^{-}\right) \longrightarrow R \otimes \mathbf{z}_{p} \mathbf{Q}_{p} .
$$


According to $(32)$, the morphism $\mathcal{L}_{\mathbb{T}}^{*}$ satisfies the desired interpolation property, which characterises it uniquely (as the kernels of the arithmetic points are dense in $\operatorname{Spec}(R)$ ).

3.3. Proof of Theorem 3.1. Write $\mathscr{R}$ for the localisation of $\bar{R}$ at $\bar{p}$, and $\mathscr{P}$ for its maximal ideal. Then $\mathscr{P}=(\varpi, \varsigma) \cdot \mathscr{R}$, where $\varpi=\gamma_{0}-1$ (resp., $\varsigma=\sigma_{0}-1$ ) is the generator of $\mathfrak{p} R_{\mathfrak{p}}$ (resp., $I$ ) fixed in (9) (resp., Section 3.1.1). Moreover the $\mathbf{Q}_{p}$-module $\mathscr{P} / \mathscr{P}^{2}$ is isomorphic to $\left(I / I^{2} \otimes \mathbf{z}_{p} \mathbf{Q}_{p}\right) \oplus\left(\mathfrak{p} R_{\mathfrak{p}} / \mathfrak{p}^{2} R_{\mathfrak{p}}\right)$.

Let $\mathfrak{Z}=\left(\mathfrak{Z}_{n}\right) \in H_{\mathrm{Iw}}^{1}\left(\mathbf{Q}_{p}, \mathbb{T}^{-}\right)$and $\mathfrak{z}:=\mathfrak{Z}_{0, \psi} \in \operatorname{Hom}_{\text {cont }}\left(\mathbf{Q}_{p}^{*}, \mathbf{Q}_{p}\right)$. According to Theorem 3.18 of [GS93]

$$
1-\mathbf{a}_{p}^{-1} \equiv-\frac{\mathscr{L}_{p}(A)}{2 \log _{p}(\varpi)} \cdot \varpi\left(\bmod \mathfrak{p}^{2} R_{\mathfrak{p}}\right)
$$

where $\log _{p}(\varpi):=\log _{p}\left(\gamma_{0}\right)$. Corollary 3.5 and Corollary 3.9 then yield the equality in $\mathscr{P} / \mathscr{P}^{2}$ :

$$
\mathcal{L}_{\mathbb{T}}(\mathfrak{Z}) \bmod \mathscr{P}^{2}=\mathcal{L}_{A}^{\prime}\left(\mathfrak{Z}_{\psi}\right)-\frac{\mathscr{L}_{p}(A)}{2 \log _{p}(\varpi)} \cdot \psi\left(\mathcal{L}_{\mathbb{T}}^{*}\left(\mathfrak{Z}_{0}\right)\right) \cdot\{\varpi\},
$$

where as usual $\{\cdot\}: \mathscr{P} \rightarrow \mathscr{P} / \mathscr{P}^{2}$ denotes the projection. Thanks to Proposition 3.6 and Proposition 3.8, the last congruence can be rewritten as

$$
\left(1-p^{-1}\right) \cdot \mathcal{L}_{\mathbb{T}}(\mathfrak{Z}) \bmod \mathscr{P}^{2}=\frac{\mathfrak{z}\left(p^{-1}\right)}{\log _{p}(\varsigma)} \cdot\{\varsigma\}-\frac{\mathscr{L}_{p}(A)}{2 \log _{p}(\varpi)} \cdot \mathfrak{z}(e(1)) \cdot\{\varpi\} .
$$

Here we used that $\psi\left(\mathbf{a}_{p}\right)=a_{p}(A)=1$ and the equality $\left\langle\exp ^{*}(\mathfrak{z}), \mho_{\psi}(1)\right\rangle_{\mathrm{dR}}=\mathfrak{z}(e(1))$. The latter follows from the definition of exp* : $H^{1}\left(\mathbf{Q}_{p}, \mathbf{Q}_{p}\right) \rightarrow D_{\mathrm{dR}}\left(\mathbf{Q}_{p}\right)=\mathbf{Q}_{p}$ (see the proof of Corollary 3.7) and our normalisation (25) of $\mho_{\psi}(1)$. Applying $\overline{\mathrm{M}}$ to both sides of the last equation, one obtains the formula displayed in Part 1 of Theorem 3.1. (Strictly speaking, the Mellin transform is defined on $\bar{R}$, but it extends to a morphism $\overline{\mathrm{M}}: \mathscr{R} \rightarrow \mathscr{M}^{\text {reg }}$, where $\mathscr{M}^{\text {reg }}$ is the localisation of $\mathscr{A}$ at the multiplicative subset $\{g(k, s) \in \mathscr{A}: g(2,1) \neq 0\}$.)

To prove Part 2 of the theorem, let $\mathfrak{Z}=\left(\mathfrak{Z}_{n}\right) \in H_{\mathrm{Iw}}^{1}\left(\mathbf{Q}_{p, \infty}, \mathbb{T}\right)$ and let $\mathfrak{z}:=\mathfrak{Z}_{0, \psi} \in H^{1}\left(\mathbf{Q}_{p}, V_{p}(A)\right)$. Since $\exp _{A}^{*}(\mathfrak{z})$ is equal to $p^{-}(\mathfrak{z})(e(1))$, using Corollary 3.7 in place of Proposition 3.6, the same argument as above yields

$$
\left(1-p^{-1}\right) \cdot \overline{\mathrm{M}} \circ \mathcal{L}_{\mathbb{T}}(\mathfrak{Z}) \equiv \mathscr{L}_{p}(A) \cdot \exp _{A}^{*}(\mathfrak{z}) \cdot(s-1)-\frac{1}{2} \mathscr{L}_{p}(A) \cdot \exp _{A}^{*}(\mathfrak{z}) \cdot(k-2)\left(\bmod \mathscr{J}^{2}\right),
$$

thus concluding the proof of Theorem 3.1.

\section{Selmer complexes and the height-weight pairing}

Inspired by Nekovář's formalism of height pairings [Nek06, Section 11], we define the height-weight pairing mentioned in the introduction. We then summarise its main properties, referring to [Nek06] and [Ven14] for the proofs.

4.1. Selmer complexes. With the notations of Section 2.7 , set $\mathfrak{G}:=\mathfrak{G}_{0}$. Let $S$ be a complete, local Noetherian ring with finite residue field of characteristic $p$, and let $\mathscr{S}$ be a localisation of $S$. Let $M=\left(M, M^{+}\right)$ be an $\mathscr{S}$-adic, nearly-ordinary representation of $\mathfrak{G}$. More precisely, $M=\mathbb{M} \otimes_{S} \mathscr{S}$ and $M^{+}=\mathbb{M}^{+} \otimes_{S} \mathscr{S}$, where $\mathbb{M}$ is a finitely generated, free $S$-module, equipped with a continuous, $S$-linear action of $\mathfrak{G}$, and $\mathbb{M}^{+} \subset \mathbb{M}^{\text {is }}$ an $S$-direct summand of $\mathbb{M}$, which is stable for the action of the decomposition group $G_{p}:=G_{\mathbf{Q}_{p}} \hookrightarrow G_{\mathbf{Q}}$ determined by the embedding $i_{p}: \overline{\mathbf{Q}} \hookrightarrow \overline{\mathbf{Q}}_{p}$.

For every prime $q \mid N$, fix an embedding $i_{q}: \overline{\mathbf{Q}} \hookrightarrow \overline{\mathbf{Q}}_{q}$, and write $G_{q}:=G_{\mathbf{Q}_{q}} \hookrightarrow G_{\mathbf{Q}}$ for the corresponding decomposition group at $q$. Following [Nek06], define Nekováŕ's Selmer complex of $M$ as the complex of $S$-modules:

$$
\widetilde{C}_{f}^{\bullet}(\mathfrak{G}, M):=\text { Cone }\left(C_{\text {cont }}^{\bullet}(\mathfrak{G}, M) \oplus C_{\text {cont }}^{\bullet}\left(\mathbf{Q}_{p}, M^{+}\right) \stackrel{\operatorname{res}_{N p}-i^{+}}{\longrightarrow} \bigoplus_{l \mid N p} C_{\text {cont }}^{\bullet}\left(\mathbf{Q}_{l}, M\right)\right)[-1],
$$

where the notations are as follows. For $G=\mathfrak{G}$ or $G=G_{l}(l \mid N p), C_{\text {cont }}^{\bullet}(G, \star)$ is the complex of continuous (non-homogeneous) cochains of $G$ with values in $\star$ and $C_{\text {cont }}^{\bullet}\left(\mathbf{Q}_{l}, \star\right):=C_{\text {cont }}^{\bullet}\left(G_{l}, \star\right)$ (see Section 3 of $[\mathbf{N e k 0 6}]$ ). $i^{+}: C_{\text {cont }}^{\bullet}\left(\mathbf{Q}_{p}, M^{+}\right) \rightarrow C_{\text {cont }}^{\bullet}\left(\mathbf{Q}_{p}, M\right)$ is the morphism induced by $M^{+} \subset M$. Finally, for every prime $l \mid N p$, $\operatorname{res}_{l}: C_{\text {cont }}^{\bullet}(\mathfrak{G}, M) \rightarrow C_{\text {cont }}^{\bullet}\left(\mathbf{Q}_{l}, M\right)$ is the restriction morphism associated with the decomposition group $G_{l} \hookrightarrow G_{\mathbf{Q}}$ and $\operatorname{res}_{N p}$ is the direct sum of the morphisms $\operatorname{res}_{l}$, for $l \mid N p$.

Denote by $\mathrm{D}(\mathscr{S})$ the derived category of complexes of $\mathscr{S}$-modules and by $\mathrm{D}(\mathscr{S})_{\mathrm{ft}}^{b} \subset \mathrm{D}(\mathscr{S})$ the subcategory of cohomologically bounded complexes with cohomology of finite type over $\mathscr{S}$. Write

$$
\widetilde{\mathbf{R}}_{f}(\mathbf{Q}, M) \in \mathrm{D}(\mathscr{S})_{\mathrm{ft}}^{b} ; \quad \widetilde{H}_{f}^{*}(\mathbf{Q}, M):=H^{*}\left(\widetilde{\mathbf{R}}_{f}(\mathbf{Q}, M)\right)
$$

for the image of $\widetilde{C}_{f}^{\bullet}(\mathfrak{G}, M)$ in $\mathrm{D}(\mathscr{S})_{\mathrm{ft}}^{b}$ and its cohomology respectively. 
By construction, there is an exact triangle in $\mathrm{D}(\mathscr{S})_{\mathrm{ft}}^{b}$ (cf. Section 6 of [Nek06]):

$$
\widetilde{\mathbf{R}}_{f}(\mathbf{Q}, M) \longrightarrow \mathbf{R} \Gamma_{\text {cont }}(\mathfrak{G}, M) \longrightarrow \mathbf{R} \Gamma_{\text {cont }}\left(\mathbf{Q}_{p}, M^{-}\right) \oplus \bigoplus_{l \mid N} \mathbf{R} \Gamma_{\text {cont }}\left(\mathbf{Q}_{l}, M\right),
$$

which gives rise to a long exact cohomology sequence of $\mathscr{S}$-modules

$$
\cdots \rightarrow H^{q-1}\left(\mathbf{Q}_{p}, M^{-}\right) \oplus H_{N}^{q-1}(M) \rightarrow \widetilde{H}_{f}^{q}(\mathbf{Q}, M) \rightarrow H^{q}(\mathfrak{G}, M) \rightarrow H^{q}\left(\mathbf{Q}_{p}, M^{-}\right) \oplus H_{N}^{q}(M) \rightarrow \cdots .
$$

Here $M^{-}:=M / M^{+}=\mathbb{M} / \mathbb{M}^{+} \otimes_{S} \mathscr{S}, \mathbf{R} \Gamma_{\text {cont }}(G, \star) \in \mathrm{D}(\mathscr{S})_{\mathrm{ft}}^{b}$ is the image of $C_{\text {cont }}^{\bullet}(G, \star)$ in the derived category, and we write for simplicity $H_{N}^{q}(M):=\bigoplus_{l \mid N} H^{q}\left(\mathbf{Q}_{l}, M\right)$.

4.2. The extended Selmer group. Let $\mathscr{S}=\mathbf{Q}_{p}$ and $M=V_{p}(A)$, with the nearly-ordinary structure $i^{+}: \mathbf{Q}_{p}(1) \hookrightarrow V_{p}(A)$ given in (14). By [Nek06, 12.5.9.2], one can extract from (33) a short exact sequence of $\mathbf{Q}_{p}$-modules

$$
0 \rightarrow \mathbf{Q}_{p} \rightarrow \widetilde{H}_{f}^{1}\left(\mathbf{Q}, V_{p}(A)\right) \rightarrow H_{f}^{1}\left(\mathbf{Q}, V_{p}(A)\right) \rightarrow 0,
$$

where the left-most term arises as $H^{0}\left(\mathbf{Q}_{p}, \mathbf{Q}_{p}\right)=H^{0}\left(\mathbf{Q}_{p}, V_{p}(A)^{-}\right)$and $H_{f}^{1}\left(\mathbf{Q}, V_{p}(A)\right) \subset H^{1}\left(\mathfrak{G}, V_{p}(A)\right)$ is the Bloch-Kato Selmer group of $V_{p}(A)$ [BK90]. In addition the projection in (34) admits a natural splitting

$$
\sigma^{\mathrm{u}-\mathrm{r}}: H_{f}^{1}\left(\mathbf{Q}, V_{p}(A)\right) \longrightarrow \widetilde{H}_{f}^{1}\left(\mathbf{Q}, V_{p}(A)\right),
$$

characterised by the following property. Let $\wp^{+}: \widetilde{H}_{f}^{1}\left(\mathbf{Q}, V_{p}(A)\right) \rightarrow H^{1}\left(\mathbf{Q}_{p}, \mathbf{Q}_{p}(1)\right)=\mathbf{Q}_{p}^{*} \widehat{\otimes} \mathbf{Q}_{p}$ be the morphism induced by the natural projection $\widetilde{\mathbf{R}}_{f}\left(\mathbf{Q}, V_{p}(A)\right) \rightarrow \mathbf{R} \Gamma_{\text {cont }}\left(\mathbf{Q}_{p}, \mathbf{Q}_{p}(1)\right)$. Then

$$
\wp^{+} \circ \sigma^{\mathrm{u}-\mathrm{r}}\left(H_{f}^{1}\left(\mathbf{Q}, V_{p}(A)\right)\right) \subset H_{f}^{1}\left(\mathbf{Q}_{p}, \mathbf{Q}_{p}(1)\right)=\mathbf{Z}_{p}^{*} \widehat{\otimes} \mathbf{Q}_{p} .
$$

This follows from Section 11.4 of [Nek06], thanks to the fact that $\mathscr{L}_{p}(A) \neq 0$ by [BSDGP96]. We use the section $\sigma^{\mathrm{u}-\mathrm{r}}$ to obtain the identification $\widetilde{H}_{f}^{1}\left(\mathbf{Q}, V_{p}(A)\right) \cong \mathbf{Q}_{p} \oplus H_{f}^{1}\left(\mathbf{Q}, V_{p}(A)\right)$. Moreover, we identify the Tate period $q_{A}$ with the canonical generator of $\mathbf{Q}_{p} \subset \widetilde{H}_{f}^{1}\left(\mathbf{Q}, V_{p}(A)\right)$. In other words, from now on

$$
\widetilde{H}_{f}^{1}\left(\mathbf{Q}, V_{p}(A)\right)=\mathbf{Q}_{p} \cdot q_{A} \oplus H_{f}^{1}\left(\mathbf{Q}, V_{p}(A)\right) .
$$

4.3. The height-weight pairing. As in Section 3.3 , let $\mathscr{R}$ be the localisation of $\bar{R}=R \llbracket G_{\infty} \rrbracket$ at $\overline{\mathfrak{p}}=(\mathfrak{p}, I)$ and let $\mathscr{P}=(\varpi, \varsigma) \cdot \mathscr{R}$ be its maximal ideal. Let $\mathscr{M}^{\text {reg }} \subset \operatorname{Frac}(\mathscr{A})$ be the localisation of $\mathscr{A}$ at the multiplicative subset consisting of elements $g(k, s) \in \mathscr{A}$ such that $g(2,1) \neq 0$, and write again $\mathscr{J} \subset \mathscr{M}^{\text {reg }}$ for the ideal of functions vanishing at $(2,1)$. The Mellin transform extends to a morphism $\overline{\mathrm{M}}: \mathscr{R} \rightarrow \mathscr{M}^{\text {reg }}$ mapping $\mathscr{P}$ into $\mathscr{J}$ and then induces a morphism of $\mathbf{Q}_{p}$-modules $\overline{\mathrm{M}}: \mathscr{P} / \mathscr{P}^{2} \rightarrow \mathscr{J} / \mathscr{J}^{2}$.

Denote by $\chi_{\infty}: \mathfrak{G} \rightarrow G_{\infty} \subset \bar{R}^{*}$ the tautological representation of $\mathfrak{G}$ and define

$$
\overline{\mathbb{T}}:=\mathbb{T} \otimes_{R} \bar{R}\left(\chi_{\infty}^{-1}\right) \in \bar{R}_{[\mathfrak{G}]} \operatorname{Mod} ; \quad T:=\overline{\mathbb{T}} \otimes_{\bar{R}} \mathscr{R} \in \mathscr{R}[\mathfrak{G}]^{M o d} .
$$

Similarly, define the $\bar{R}\left[G_{p}\right]$-modules $\overline{\mathbb{T}}^{ \pm}:=\mathbb{T}^{ \pm} \otimes_{R} \bar{R}\left(\chi_{\infty}^{-1}\right)$ and the $\mathscr{R}\left[G_{p}\right]$-modules $T^{ \pm}:=\overline{\mathbb{T}}^{ \pm} \otimes_{R} \mathscr{R}$. Then $\overline{\mathbb{T}}^{ \pm}$are free $\bar{R}$-modules of rank one, so that $T=\left(T, T^{+}\right)$is a nearly-ordinary $\mathscr{R}$-adic representation of $\mathfrak{G}$. In particular, there is a short exact sequence of $\mathscr{R}\left[G_{p}\right]$-modules

$$
0 \longrightarrow T^{+} \stackrel{i^{+}}{\longrightarrow} T \stackrel{p^{-}}{\longrightarrow} T^{-} \longrightarrow 0
$$

and the Selmer complex $\widetilde{\mathbf{R}}_{f}(\mathbf{Q}, T) \in \mathrm{D}(\mathscr{R})_{\mathrm{ft}}^{b}$ is defined.

Denote by $\xi: \mathscr{R} \rightarrow \mathbf{Q}_{p}$ the composition of $\psi: R_{\mathfrak{p}} \rightarrow \mathbf{Q}_{p}$ with the augmentation map $\varepsilon: \mathscr{R} \rightarrow R_{\mathfrak{p}}$. Since $\varepsilon \circ \chi_{\infty}$ is the trivial character, (13) induces a natural isomorphism of $\mathbf{Q}_{p}[\mathfrak{G}]$-modules

$$
T_{\xi}:=T \otimes_{\mathscr{R}, \xi} \mathbf{Q}_{p} \cong V_{p}(A) .
$$

Similarly $T_{\xi}^{+}:=T^{+} \otimes_{\mathscr{R}, \xi} \mathbf{Q}_{p} \cong \mathbf{Q}_{p}(1)$ and $T_{\xi}^{-}:=T^{-} \otimes_{\mathscr{R}, \xi} \mathbf{Q}_{p} \cong \mathbf{Q}_{p}$ as $\mathbf{Q}_{p}\left[G_{p}\right]$-modules, and (38) extends to an isomorphism between the $\xi$-base change of (37) and the tensor product of (14) with $\mathbf{Q}_{p}$. This induces a canonical isomorphism of complexes of $\mathbf{Q}_{p}$-modules

$$
\widetilde{\mathbf{R}}_{f}\left(\mathbf{Q}, T_{\xi}\right) \cong \widetilde{\mathbf{R}}_{f}\left(\mathbf{Q}, V_{p}(A)\right)
$$


4.3.1. The Bockstein map. By the general behaviour of Selmer complexes under base change, $\widetilde{\mathbf{R} \Gamma} f\left(\mathbf{Q}, T_{\xi}\right)$ is isomorphic to the derived base change $\widetilde{\mathbf{R}}_{f}(\mathbf{Q}, T) \otimes_{\mathscr{R}, \xi}^{\mathbf{L}} \mathbf{Q}_{p}$. This yields via (39) natural isomorphisms in $\mathrm{D}(\mathscr{R})_{\mathrm{ft}}^{b}$ :

$$
\widetilde{\mathbf{R}}_{f}(\mathbf{Q}, T) \otimes \otimes_{\mathscr{R}, \xi}^{\mathbf{L}} \mathbf{Q}_{p} \cong \widetilde{\mathbf{R}}_{f}\left(\mathbf{Q}, V_{p}(A)\right) ; \quad \widetilde{\mathbf{R}}_{f}(\mathbf{Q}, T) \otimes \mathscr{\mathscr { R }}_{\mathscr{R}}^{\mathbf{L}} / \mathscr{P}^{2} \cong \widetilde{\mathbf{R}}_{f}\left(\mathbf{Q}, V_{p}(A)\right) \otimes_{\mathbf{Q}_{p}} \mathscr{P} / \mathscr{P}^{2} .
$$

(For the details see the proof of Lemma 5.5 below; see also the proof of Proposition 8.10.1 of [Nek06].) Applying the functor $\widetilde{\mathbf{R}}_{f}(\mathbf{Q}, T) \otimes \mathbb{R}_{\mathscr{R}}-$ to the exact triangle

$$
\mathscr{P} / \mathscr{P}^{2} \longrightarrow \mathscr{R} / \mathscr{P}^{2} \stackrel{\xi}{\longrightarrow} \mathbf{Q}_{p} \stackrel{\partial_{\xi}}{\longrightarrow} \mathscr{P} / \mathscr{P}^{2}[1]
$$

then induces a morphism in $\mathrm{D}(\mathscr{R})_{\mathrm{ft}}^{b}$ :

$$
\widetilde{\boldsymbol{\beta}}_{p}: \widetilde{\mathbf{R}}_{f}\left(\mathbf{Q}, V_{p}(A)\right) \longrightarrow \widetilde{\mathbf{R}}_{f}\left(\mathbf{Q}, V_{p}(A)\right)[1] \otimes_{\mathbf{Q}_{p}} \mathscr{P} / \mathscr{P}^{2}
$$

called the derived Bockstein map. It induces in cohomology the Bockstein map

$$
\widetilde{\beta}_{p}:=H^{1}\left(\widetilde{\boldsymbol{\beta}}_{p}\right): \widetilde{H}_{f}^{1}\left(\mathbf{Q}, V_{p}(A)\right) \longrightarrow \widetilde{H}_{f}^{2}\left(\mathbf{Q}, V_{p}(A)\right) \otimes_{\mathbf{Q}_{p}} \mathscr{P} / \mathscr{P}^{2} .
$$

4.3.2. Definition of the pairing. Nekovář's generalisation of Poitou-Tate duality attaches to the Weil pairing on $V_{p}(A)$ a perfect, global cup-product pairing [Nek06, Section 6]

$$
\langle-,-\rangle_{\mathrm{Nek}}: \widetilde{H}_{f}^{2}\left(\mathbf{Q}, V_{p}(A)\right) \otimes \mathbf{Q}_{p} \widetilde{H}_{f}^{1}\left(\mathbf{Q}, V_{p}(A)\right) \longrightarrow H_{c, \text { cont }}^{3}\left(\mathbf{Q}, \mathbf{Q}_{p}(1)\right) \cong \mathbf{Q}_{p},
$$

where $H_{c, \text { cont }}^{*}(\mathbf{Q},-)$ denotes the compactly supported cohomology and the last trace isomorphism comes from global class field theory [Nek06, Section 5]. (See in particular Sections 5.3.1.3, 5.4.1 and 6.3 of [Nek06].)

We define the (cyclotomic) height-weight pairing

$$
\langle-,-\rangle_{p}: \widetilde{H}_{f}^{1}\left(\mathbf{Q}, V_{p}(A)\right) \otimes_{\mathbf{Q}_{p}} \widetilde{H}_{f}^{1}\left(\mathbf{Q}, V_{p}(A)\right) \longrightarrow \mathscr{J} / \mathscr{J}^{2}
$$

as the composition of

$$
\widetilde{\beta}_{p} \otimes \mathrm{id}: \widetilde{H}_{f}^{1}\left(\mathbf{Q}, V_{p}(A)\right) \otimes_{\mathbf{Q}_{p}} \widetilde{H}_{f}^{1}\left(\mathbf{Q}, V_{p}(A)\right) \longrightarrow \widetilde{H}_{f}^{2}\left(\mathbf{Q}, V_{p}(A)\right) \otimes_{\mathbf{Q}_{p}} \widetilde{H}_{f}^{1}\left(\mathbf{Q}, V_{p}(A)\right) \otimes_{\mathbf{Q}_{p}} \mathscr{P} / \mathscr{P}^{2}
$$

with

$$
\langle-,-\rangle_{\mathrm{Nek}} \otimes \overline{\mathrm{M}}: \widetilde{H}_{f}^{2}\left(\mathbf{Q}, V_{p}(A)\right) \otimes_{\mathbf{Q}_{p}} \widetilde{H}_{f}^{1}\left(\mathbf{Q}, V_{p}(A)\right) \otimes_{\mathbf{Q}_{p}} \mathscr{P} / \mathscr{P}^{2} \longrightarrow \mathscr{J} / \mathscr{J}^{2} .
$$

We also write $\langle-,-\rangle\rangle_{p}(k, s):=\left\langle\langle-,-\rangle_{p}\right.$ when we want to emphasise the dependence of $\langle-,-\rangle_{p}$ on the variables $(k, s)$. If $F: \mathscr{M}^{\text {reg }} \rightarrow \mathscr{M}^{\text {reg }}$ is a morphism of $\mathbf{Q}_{p}$-algebras s.t. $F(\mathscr{J}) \subset \mathscr{J}$, then $\langle-,-\rangle_{p}(F(k, s)):=F \circ\left\langle\langle-,-\rangle_{p}\right.$.

REMARK 4.1. Let $W: V_{p}(A) \otimes \mathbf{Q}_{p} V_{p}(A) \rightarrow \mathbf{Q}_{p}(1)$ be the Weil pairing, normalised as in [Sil86, Chapter III]. In order to define $\langle-,-\rangle_{p}$ without ambiguities, one has to fix the Tate parametrisation $\Phi_{\text {Tate }}$ introduced in (3), which is unique up to sign. We do this by requiring: $W\left(a, i^{+}(b)\right)=p^{-}(a) \cdot b$ for every $a \in V_{p}(A)$ and $b \in \mathbf{Q}_{p}(1)$.

4.3.3. Basic properties. In this section we discuss the basic properties satisfied by the height-weight pairing, referring to [Nek06, Section 11] and [Ven14] for the proofs.

Section 7 of [Nek93] defines a symmetric (cyclotomic) canonical height pairing

$$
\langle-,-\rangle_{p}^{c y c}: H_{f}^{1}\left(\mathbf{Q}, V_{p}(A)\right) \otimes_{\mathbf{Q}_{p}} H_{f}^{1}\left(\mathbf{Q}, V_{p}(A)\right) \longrightarrow \mathbf{Q}_{p},
$$

denoted $h^{\text {can }}$ in [Nek93]. More precisely, after identifying $V_{p}(A)$ with its Kummer dual under the Weil pairing, the definition of $h^{\text {can }}$ rests on the choices of a continuous morphism $\lambda_{p}: \mathbb{A}_{\mathbf{Q}}^{*} / \mathbf{Q}^{*} \rightarrow \mathbf{Q}_{p}$ (where $\mathbb{A}_{\mathbf{Q}}^{*}$ is the group of ideles of $\mathbf{Q}$ ) and a splitting sp : $D_{\mathrm{dR}}\left(V_{p}(A)\right) \rightarrow \mathrm{Fil}^{0} D_{\mathrm{dR}}\left(V_{p}(A)\right)$ of the natural filtration. In the definition of $\langle-,-\rangle_{p}^{\text {cyc }}$, $\lambda_{p}$ is the composition of the Artin map $\mathbb{A}_{\mathbf{Q}}^{*} / \mathbf{Q}^{*} \rightarrow G_{\mathbf{Q}}^{\mathrm{ab}}$ with $\log _{p} \circ \chi_{\mathrm{cyc}}: G_{\mathbf{Q}}^{\mathrm{ab}} \rightarrow \mathbf{Q}_{p}$, and sp is the splitting induced by (24). Let $\{\cdot\}: \mathscr{J} \rightarrow \mathscr{J} / \mathscr{J}^{2}$ denote the projection. Given $g(k, s)=a \cdot\{s-1\}+b \cdot\{k-2\} \in \mathscr{J} / \mathscr{J}^{2}$, write $\frac{d}{d s} g(2, s)_{s=1}:=a$ and $\frac{d}{d k} g(k, 1)_{k=2}:=b$.

THEOREM 4.2. The $\mathbf{Q}_{p}$-bilinear form $\langle-,-\rangle_{p}$ enjoys the following properties.

1. (Cyclotomic specialisation) For every $x, y \in H_{f}^{1}\left(\mathbf{Q}, V_{p}(A)\right)$ :

$$
\frac{d}{d s}\left(\left\langle\langle x, y\rangle_{p}(2, s)\right)_{s=1}=\langle x, y\rangle_{p}^{\mathrm{cyc}} .\right.
$$

2. (Exceptional zero formulae) For every $z \in H_{f}^{1}\left(\mathbf{Q}, V_{p}(A)\right)$ :

$$
\left\langle\left\langle q_{A}, q_{A}\right\rangle\right\rangle_{p}=\log _{p}\left(q_{A}\right) \cdot\{s-k / 2\} ;\left\langle\left\langle q_{A}, z\right\rangle_{p}=\log _{A}\left(\operatorname{res}_{p}(z)\right) \cdot\{s-1\},\right.
$$

where $\log _{A}=\log _{q_{A}} \circ \Phi_{\text {Tate }}^{-1}: H_{f}^{1}\left(\mathbf{Q}_{p}, V_{p}(A)\right) \cong A\left(\mathbf{Q}_{p}\right) \widehat{\otimes} \mathbf{Q}_{p} \rightarrow \mathbf{Q}_{p}$ is the formal group logarithm.

3. (Functional equation) For every $x, y \in \widetilde{H}_{f}^{1}\left(\mathbf{Q}, V_{p}(A)\right)$ :

$$
\left\langle\langle y, x\rangle_{p}(k, s)=-\left\langle\langle x, y\rangle_{p}(k, k-s) .\right.\right.
$$

Proof. Part 1 is proved in [Nek06, Corollary 11.4.7]. Part 2 and Part 3 are proved in [Ven14]. 


\section{Exceptional zero formulae à la Rubin}

Recall the extended height-weight $\widetilde{h}_{p}: H_{f}^{1}\left(\mathbf{Q}, V_{p}(A)\right) \rightarrow \mathscr{J}^{2} / \mathscr{J}^{3}$ introduced in (4). For every $\mathfrak{Z}=\left(\mathfrak{Z}_{n}\right) \in$ $H_{\mathrm{Iw}}^{1}\left(\mathbf{Q}_{\infty}, \mathbb{T}\right)$, write $\mathfrak{Z}_{n, \psi} \in H^{1}\left(\mathfrak{G}_{n}, V_{p}(A)\right)$ for the image of $\mathfrak{Z}_{n}$ under the morphism induced by $\mathbb{T} \rightarrow \mathbb{T}_{\psi} \subset V_{p}(A)$. The aim of this section is to prove the following theorem, reminiscent of the Rubin formulae proved by Rubin [Rub94] and Perrin-Riou [PR93, Section 2.3] in a different setting (see also [Nek06, Sec. 11]).

Theorem 5.1. Let $\mathfrak{Z}=\left(\mathfrak{Z}_{n}\right) \in H_{\mathrm{Iw}}^{1}\left(\mathbf{Q}_{\infty}, \mathbb{T}\right)$ and let $\mathfrak{z}:=\mathfrak{Z}_{0, \psi} \in H^{1}\left(\mathfrak{G}, V_{p}(A)\right)$.

1. We have the equality in $\mathscr{J} / \mathscr{J}^{2}$ :

$$
\mathcal{L}_{\mathbb{T}}\left(\operatorname{res}_{p}(\mathfrak{Z}), k, s\right) \bmod \mathscr{J}^{2}=\frac{1}{\operatorname{ord}_{p}\left(q_{A}\right)}\left(1-\frac{1}{p}\right)^{-1} \exp _{A}^{*}\left(\operatorname{res}_{p}(\mathfrak{z})\right) \cdot\left\langle\left\langle q_{A}, q_{A}\right\rangle_{p} \cdot\right.
$$

In particular: $\mathcal{L}_{\mathbb{T}}\left(\operatorname{res}_{p}(\mathfrak{Z}), k, s\right) \in \mathscr{J}^{2}$ if and only if $\mathfrak{z} \in H_{f}^{1}\left(\mathbf{Q}, V_{p}(A)\right)$.

2. If $\mathfrak{z} \in H_{f}^{1}\left(\mathbf{Q}, V_{p}(A)\right)$, we have the equality in $\mathscr{J}^{2} / \mathscr{J}^{3}$ :

$$
\log _{A}\left(\operatorname{res}_{p}(\mathfrak{z})\right) \cdot \mathcal{L}_{\mathbb{T}}\left(\operatorname{res}_{p}(\mathfrak{Z}), k, s\right) \bmod \mathscr{J}^{3}=\frac{-1}{\operatorname{ord}_{p}\left(q_{A}\right)}\left(1-\frac{1}{p}\right)^{-1} \cdot \widetilde{h}_{p}(\mathfrak{z}) .
$$

This result, whose proof is given in Section 5.2 below, becomes particularly relevant when combined with the work of Kato. Recall the class $\mathfrak{Z}_{\infty}^{\mathrm{BK}} \in H_{\mathrm{Iw}}^{1}\left(\mathbf{Q}_{\infty}, \mathbb{T}\right)$ appearing in Theorem 2.4. By loc. cit. and equation (20)

$$
\mathcal{L}_{\mathbb{T}}\left(\operatorname{res}_{p}\left(\mathfrak{Z}_{\infty}^{\mathrm{BK}}\right), k, s\right)=L_{p}\left(f_{\infty}, k, s\right) .
$$

With the notations of the introduction, we set

$$
\zeta_{\infty}^{\mathrm{BK}}:=\mathfrak{Z}_{\infty, \psi}^{\mathrm{BK}} \in H_{\mathrm{Iw}}^{1}\left(\mathbf{Q}_{\infty}, T_{p}(A)\right) ; \quad \zeta^{\mathrm{BK}}=\mathfrak{Z}_{0, \psi}^{\mathrm{BK}} \in H^{1}\left(\mathfrak{G}, V_{p}(A)\right) .
$$

By Corollary 3.5 and equation $(17), \mathcal{L}_{A}\left(\operatorname{res}_{p}\left(\zeta_{\infty}^{\mathrm{BK}}\right)\right)=L_{p}(A / \mathbf{Q})$; this is equation (1) in the introduction.

Equation (42) and Theorem 5.1(1) yield the following result, which in light of Kato's reciprocity law (2) and Theorem 4.2(2) can be seen as a variant of the main result of [GS93].

THeOREM 5.2. We have the equality in $\mathscr{J} / \mathscr{J}^{2}$ :

$$
L_{p}\left(f_{\infty}, k, s\right) \bmod \mathscr{J}^{2}=\frac{1}{\operatorname{ord}_{p}\left(q_{A}\right)}\left(1-\frac{1}{p}\right)^{-1} \exp _{A}^{*}\left(\operatorname{res}_{p}\left(\zeta^{\mathrm{BK}}\right)\right) \cdot\left\langle\left\langle q_{A}, q_{A}\right\rangle_{p} .\right.
$$

In particular, $L_{p}\left(f_{\infty}, k, s\right) \in \mathscr{J}^{2}$ if and only if $\zeta^{\mathrm{BK}}$ is a Selmer class.

Theorem 5.1(2) and (42) combine to give the following theorem (cf. Section 1.3).

Theorem 5.3. Assume that $\zeta^{\mathrm{BK}} \in H_{f}^{1}\left(\mathbf{Q}, V_{p}(A)\right)$. Then we have the equality in $\mathscr{J}^{2} / \mathscr{J}^{3}$ :

$$
\log _{A}\left(\operatorname{res}_{p}\left(\zeta^{\mathrm{BK}}\right)\right) \cdot L_{p}\left(f_{\infty}, k, s\right) \bmod \mathscr{J}^{3}=\frac{-1}{\operatorname{ord}_{p}\left(q_{A}\right)}\left(1-\frac{1}{p}\right)^{-1} \cdot \widetilde{h}_{p}\left(\zeta^{\mathrm{BK}}\right) .
$$

5.1. Derivatives of cohomology classes. With the notations of Section 4.3, Shapiro's Lemma gives a natural isomorphism of $\mathscr{R}$-modules

$$
H^{1}\left(\mathbf{Q}_{p}, T^{-}\right) \cong H_{\mathrm{Iw}}^{1}\left(\mathbf{Q}_{p, \infty}, \mathbb{T}^{-}\right) \otimes_{\bar{R}} \mathscr{R},
$$

under which the morphism $\xi_{*}: H^{1}\left(\mathbf{Q}_{p}, T^{-}\right) \rightarrow H^{1}\left(\mathbf{Q}_{p}, \mathbf{Q}_{p}\right)$ induced by $T^{-} \rightarrow T_{\xi}^{-} \cong \mathbf{Q}_{p}$ (see (38)) corresponds to the $\mathscr{R}$-base change of $H_{\mathrm{Iw}}^{1}\left(\mathbf{Q}_{p, \infty}, \mathbb{T}^{-}\right) \rightarrow H^{1}\left(\mathbf{Q}_{p}, \mathbf{Q}_{p}\right) ;\left(\mathfrak{Z}_{n}\right) \mapsto \mathfrak{Z}_{0, \psi}$. Under this isomorphism, $\mathcal{L}_{\mathbb{T}}(\cdot, k, s)$ gives rise to a morphism of $\mathscr{R}$-modules (denoted again by the same symbol)

$$
\mathcal{L}_{\mathbb{T}}(\cdot, k, s): H^{1}\left(\mathbf{Q}_{p}, T^{-}\right) \longrightarrow \mathscr{J} \subset \mathscr{M}^{\mathrm{reg}} .
$$

As usual, one writes $\mathcal{L}_{\mathbb{T}}(\cdot, k, s): H^{1}\left(\mathbf{Q}_{p}, T\right) \rightarrow \mathscr{J}$ also for the morphism induced by the projection $p^{-}: T \rightarrow T^{-}$.

Denote by $H^{1}\left(\mathbf{Q}_{p}, T\right)^{o} \subset H^{1}\left(\mathbf{Q}_{p}, T\right)$ the submodule consisting of classes $\mathfrak{Y}$ such that $p^{-}(\mathfrak{Y}) \in \mathscr{P} \cdot H^{1}\left(\mathbf{Q}_{p}, T^{-}\right)$. Given $\mathfrak{Y} \in H^{1}\left(\mathbf{Q}_{p}, T\right)^{o}$, choose $\mathfrak{Y}_{\varpi}, \mathfrak{Y}_{\varsigma} \in H^{1}\left(\mathbf{Q}_{p}, T^{-}\right)$s.t. $p^{-}(\mathfrak{Y})=\varpi \cdot \mathfrak{Y}_{\varpi}+\varsigma \cdot \mathfrak{Y}_{\varsigma}$, write $\mathfrak{y}_{\varpi}, \mathfrak{y}_{\varsigma} \in \operatorname{Hom}_{\text {cont }}\left(\mathbf{Q}_{p}^{*}, \mathbf{Q}_{p}\right)$ for the images of $\mathfrak{Y}_{\varpi}, \mathfrak{Y}_{\varsigma}$ under $\xi_{*}$ and define

$$
\begin{gathered}
\operatorname{Der}_{\mathrm{wt}}(\mathfrak{Y}):=\log _{p}(\varpi) \cdot \mathfrak{y}_{\varpi}(e(1)) ; \quad \operatorname{Der}_{\mathrm{cyc}}(\mathfrak{Y}):=\log _{p}(\varsigma) \cdot \mathfrak{y}_{\varsigma}\left(p^{-1}\right) ; \\
\operatorname{Der}_{\dagger}(\mathfrak{Y}):=\log _{p}(\varpi) \cdot \mathfrak{y}_{\varpi}\left(p^{-1}\right)-\frac{1}{2} \log _{p}(\varsigma) \cdot \mathscr{L}_{p}(A) \cdot \mathfrak{y}_{\varsigma}(e(1)),
\end{gathered}
$$

where $\log _{p}(\varpi):=\log _{p}\left(\gamma_{0}\right)$ and $\log _{p}(\varsigma):=\log _{p}\left(\chi_{\text {cyc }}\left(\sigma_{0}\right)\right)$. Note that, for $* \in\{$ wt, cyc, $\dagger\}$, the definition of $\operatorname{Der}_{*}(\mathfrak{Y})$ depends a priori on the choice of the classes $\mathfrak{Y}_{\varpi}$ and $\mathfrak{Y}_{\varsigma}$. That it is indeed independent of this choice is a consequence of the following corollary of Theorem 3.1 and the non-vanishing of $\mathscr{L}_{p}(A)$. 
Corollary 5.4. For every $\mathfrak{Y} \in H^{1}\left(\mathbf{Q}_{p}, T\right)^{\circ}$, we have

$$
\left(1-\frac{1}{p}\right) \mathcal{L}_{\mathbb{T}}(\mathfrak{Y}, k, s) \equiv \operatorname{Der}_{\text {cyc }}(\mathfrak{Y}) \cdot(s-1)^{2}+\operatorname{Der}_{\dagger}(\mathfrak{Y}) \cdot(s-1)(k-2)-\frac{1}{2} \mathscr{L}_{p}(A) \cdot \operatorname{Der}_{\mathrm{wt}}(\mathfrak{Y}) \cdot(k-2)^{2}\left(\bmod \mathscr{J}^{3}\right) .
$$

Proof. As $\log _{p}(\varpi)(k-2)$ and $\log _{p}(\varsigma)(s-1)$ are the linear terms of $\overline{\mathrm{M}}(\varpi)$ and $\overline{\mathrm{M}}(\varsigma)$ respectively, and $\mathcal{L}_{\mathbb{T}}(\cdot, k, s)$ factorises through an $\mathscr{R}$-linear map on $H^{1}\left(\mathbf{Q}_{p}, T^{-}\right)$, this is a direct consequence of Theorem 3.1(1).

5.2. Proof of Theorem 5.1. Part 1 of the theorem follows by combining Theorem 3.1(2) with Theorem 4.2(2). We then concentrate on the proof of Part 2 in the rest of this section.

Notations. With the notations of Section 4.1 , set $\widetilde{C}_{f}^{\bullet}(M):=\widetilde{C}_{f}^{\bullet}(\mathfrak{G}, M)$. Write $\widetilde{x}=\left(x, x^{+}, y\right)$ for an $n$ cochain of $\widetilde{C}_{f}^{\bullet}(M)$, where $x \in C_{\text {cont }}^{n}(\mathfrak{G}, M), x^{+} \in C_{\text {cont }}^{n}\left(\mathbf{Q}_{p}, M^{+}\right)$and $y=\left(y_{l}\right)_{l \mid N p} \in \bigoplus_{l \mid N p} C_{\text {cont }}^{n-1}\left(\mathbf{Q}_{l}, M\right)$. Denote by $\widetilde{d}$ the differentials of $\widetilde{C}_{f}^{\bullet}(M)$, so that $\widetilde{d}(\widetilde{x})=\left(d(x), d\left(x^{+}\right), i^{+}\left(x^{+}\right)-\operatorname{res}_{N p}(x)-d(y)\right)$, where the $d$ 's are the differentials of $C_{\text {cont }}^{\bullet}(-,-)$. Write $\xi_{*}: \widetilde{C}_{f}^{\bullet}(T) \rightarrow \widetilde{C}_{f}^{\bullet}\left(V_{p}(A)\right), \xi_{*}: C_{\text {cont }}^{\bullet}(\mathfrak{G}, T) \rightarrow C_{\text {cont }}^{\bullet}\left(\mathfrak{G}, V_{p}(A)\right)$ and $\xi_{*}: C_{\text {cont }}^{\bullet}\left(\mathbf{Q}_{p}, T^{?}\right) \rightarrow C_{\text {cont }}^{\bullet}\left(\mathbf{Q}_{p}, V_{p}(A)^{?}\right.$ ) (with ? $\in\{\emptyset, \pm\}$ ) to denote the morphisms induced on cochains by $T \rightarrow T_{\xi} \cong V_{p}(A)$ (38). Finally, write $\widetilde{\mathbf{R}}_{f}(M):=\widetilde{\mathbf{R}}_{f}(\mathbf{Q}, M)$ and $\widetilde{H}_{f}^{*}(M):=\widetilde{H}_{f}^{*}(\mathbf{Q}, M)$.

5.2.1. A description of $\widetilde{\beta}_{p}$. In order to prove the theorem, we need a more concrete description of the Bockstein map $\widetilde{\beta}_{p}$. This is addressed in the following lemma.

Lemma 5.5. Let $\widetilde{x} \in \widetilde{C}_{f}^{1}\left(V_{p}(A)\right)$ be a 1-cocycle, and let $\widetilde{X} \in \widetilde{C}_{f}^{1}(T)$ and $\widetilde{Y}_{\varpi}, \widetilde{Y}_{\varsigma} \in \widetilde{C}_{f}^{2}(T)$ be cochains such that:

(a) $\xi_{*}(\widetilde{X})=\widetilde{x}$

(b) $\widetilde{d}(\tilde{X})=\varpi \cdot \tilde{Y}_{\varpi}+\varsigma \cdot \tilde{Y}_{\varsigma}$.

Then $\widetilde{y}_{\varpi}:=\xi_{*}\left(\widetilde{Y}_{\varpi}\right)$ and $\widetilde{y}_{\varsigma}:=\xi_{*}\left(\widetilde{Y}_{\varsigma}\right)$ are 2 -cocycles of $\widetilde{C}_{f}^{\bullet}\left(V_{p}(A)\right)$ and

$$
-\widetilde{\beta}_{p}([\widetilde{x}])=\left[\widetilde{y}_{\varpi}\right] \otimes\{\varpi\}+\left[\widetilde{y}_{\varsigma}\right] \otimes\{\varsigma\} \in \widetilde{H}_{f}^{2}\left(V_{p}(A)\right) \otimes \mathbf{Q}_{p} \mathscr{P} / \mathscr{P}^{2}
$$

(where $[\star]$ denotes the cohomology class of $\star$, and $\{\cdot\}: \mathscr{P} \rightarrow \mathscr{P} / \mathscr{P}^{2}$ the projection).

Proof. Consider the complex of $\mathscr{R}$-modules, concentrated in degrees $(-2,0)$ :

$$
K_{\bullet}:=K_{\bullet}(\varpi, \varsigma): \mathscr{R} \stackrel{d_{2}}{\longrightarrow} \mathscr{R} \oplus \mathscr{R} \stackrel{d_{1}}{\longrightarrow} \mathscr{R},
$$

where $d_{2}(r)=(-r \varsigma, r \varpi)$ and $d_{1}(r, s)=r \varpi+s \varsigma$. It is the Koszul complex of the $\mathscr{R}$-sequence $(\varpi, \varsigma)$ generating $\mathscr{P}$. Note that the morphism $\xi$ in degree zero defines a quasi-isomorphism $\xi: K_{\bullet} \rightarrow \mathbf{Q}_{p}$. Similarly, one has a quasi-isomorphism $\xi^{\prime}: K_{\bullet}^{2} \rightarrow \mathbf{Q}_{p}^{2} \cong \mathscr{P} / \mathscr{P}^{2}$, defined in degree zero by $\xi^{\prime}(r, s)=\xi(r)\{\varpi\}+\xi(s)\{\varsigma\}$. It is then easily verified that there is a commutative diagram in $\mathrm{D}(\mathscr{R})$ :

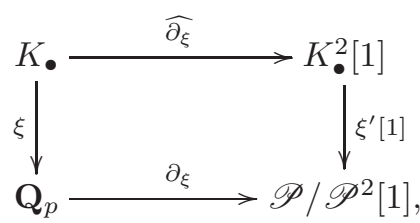

where $\partial_{\xi}$ is the morphism which appears in the exact triangle $(41)$ and $\widehat{\partial_{\xi}}$ is the morphism of complexes

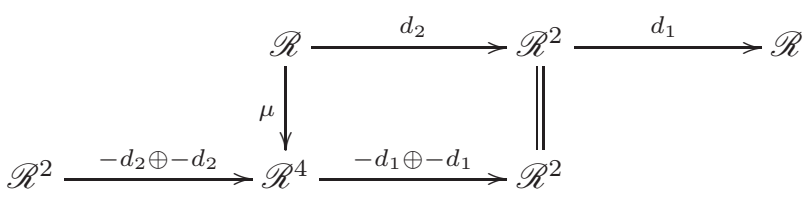

with $\mu(r):=(0, r,-r, 0)$ for every $r \in \mathscr{R}$.

As $K_{\bullet} \cong \mathbf{Q}_{p}$ in $\mathrm{D}(\mathscr{R})$ and $K_{\bullet}$ is a complex of free $\mathscr{R}$-modules, there are functorial isomorphisms in $\mathrm{D}(\mathscr{R})$ :

$$
C \otimes_{\mathscr{R}} K_{\bullet} \cong C \otimes_{\mathscr{R}, \xi}^{\mathbf{L}} \mathbf{Q}_{p} ; \quad C \otimes_{\mathscr{R}} K_{\bullet}^{2} \cong C \otimes_{\mathscr{R}}^{\mathbf{L}} \mathscr{P} / \mathscr{P}^{2}
$$

for every cohomologically bounded complex $C \in \mathrm{D}(\mathscr{R})^{b}$. Since $T$ and $T^{ \pm}$are free $\mathscr{R}$-modules, the natural projection $K_{\bullet} \rightarrow \mathscr{R} / \mathscr{P}$ (in degree zero) induces a quasi-isomorphism

$$
\widetilde{C}_{f}^{\bullet}(T) \otimes \mathscr{R} K_{\bullet} \stackrel{\text { qis }}{\longrightarrow} \widetilde{C}_{f}^{\bullet}(T) \otimes \mathscr{R} \mathscr{R} / \mathscr{P} .
$$


The complex on the right is isomorphic to $\widetilde{C}_{f}^{\bullet}\left(T_{\xi}\right) \cong \widetilde{C}_{f}^{\bullet}\left(V_{p}(A)\right)$, as follows from [Nek06, Proposition 3.4.2]. Then $\xi_{*}: \widetilde{C}_{f}^{\bullet}(T) \rightarrow \widetilde{C}_{f}^{\bullet}\left(V_{p}(A)\right)$ and (45) define a quasi-isomorphism

$$
\Xi: \widetilde{C}_{f}^{\bullet}(T) \otimes \mathscr{R} K \bullet \stackrel{\text { qis }}{\longrightarrow} \widetilde{C}_{f}^{\bullet}\left(V_{p}(A)\right),
$$

inducing via (44) the first isomorphism in (40). Similarly, consider the quasi-isomorphism

$$
\Xi^{\prime}: \widetilde{C}_{f}^{\bullet}(T) \otimes_{\mathscr{R}} K_{\bullet}^{2} \stackrel{\Xi^{2}}{\longrightarrow} \widetilde{C}_{f}^{\bullet}\left(V_{p}(A)\right) \otimes_{\mathbf{Q}_{p}} \mathbf{Q}_{p}^{2} \cong \widetilde{C}_{f}^{\bullet}\left(V_{p}(A)\right) \otimes_{\mathbf{Q}_{p}} \mathscr{P} / \mathscr{P}^{2} .
$$

The second isomorphism displayed in (40) is then induced by $\Xi^{\prime}$ via (44).

Together with (43), the preceding discussion describes the morphism $\widetilde{\beta}_{p}$ as the composition

$$
\widetilde{\beta}_{p}: \widetilde{H}_{f}^{1}\left(V_{p}(A)\right) \stackrel{\Xi_{1}^{-1}}{\longrightarrow} H^{1}\left(\widetilde{C}_{f}^{\bullet}(T) \otimes \mathscr{R} K_{\bullet}\right) \stackrel{\left(\mathrm{id} \otimes \widehat{\partial}_{\xi}\right)_{1}}{\longrightarrow} H^{2}\left(\widetilde{C}_{f}^{\bullet}(T) \otimes_{\mathscr{R}} K_{\bullet}^{2}\right) \stackrel{\Xi_{2}^{\prime}}{\longrightarrow} \widetilde{H}_{f}^{2}\left(V_{p}(A)\right) \otimes_{\mathbf{Q}_{p}} \mathscr{P} / \mathscr{P}^{2},
$$

where $(\cdot)_{n}:=H^{n}(\cdot)$. Take now $\widetilde{x}, \widetilde{X}, \widetilde{Y}_{\varpi}$ and $\widetilde{Y}_{\varsigma}$ as in the statement. The relation $(b)$ gives $\varpi \cdot \widetilde{d}\left(\widetilde{Y}_{\varpi}\right)=-\varsigma \cdot \widetilde{d}\left(\widetilde{Y}_{\varsigma}\right)$. This easily implies that $\widetilde{d}\left(\widetilde{Y}_{\varpi}\right)=\varsigma \cdot \widetilde{U}$ and $\widetilde{d}\left(\widetilde{Y}_{\varsigma}\right)=-\varpi \cdot \widetilde{U}$, for a 3-cocycle $\widetilde{U} \in \widetilde{C}_{f}^{3}(T)$. Then $(b)$ tells us that

$$
\mathbf{X}:=\left(\widetilde{U},\left(-\widetilde{Y}_{\varpi},-\widetilde{Y}_{\varsigma}\right), \widetilde{X}\right) \in \widetilde{C}_{f}^{3}(T) \oplus \widetilde{C}_{f}^{2}(T)^{2} \oplus \widetilde{C}_{f}^{1}(T)=\left(\widetilde{C}_{f}^{\bullet}(T) \otimes \mathscr{R} K_{\bullet}\right)^{1}
$$

is a 1-cocycle, and by $(a): \Xi_{1}([\mathbf{X}])=\left[\xi_{*}(\widetilde{X})\right]=[\widetilde{x}]$. Applying (id $\left.\otimes \widehat{\partial}_{\xi}\right)$ to $\mathbf{X}$ we obtain the 2-cocycle

$$
\mathbf{Y}:=\left((0, \widetilde{U},-\widetilde{U}, 0),\left(-\widetilde{Y}_{\varpi},-\widetilde{Y}_{\varsigma}\right)\right) \in \widetilde{C}_{f}^{3}(T)^{4} \oplus \widetilde{C}_{f}^{2}(T)^{2} \subset\left(\widetilde{C}_{f}^{\bullet}(T) \otimes \mathscr{R} K_{\bullet}^{2}\right)^{2} .
$$

By equation (46) one has $\widetilde{\beta}_{p}([\widetilde{x}])=\Xi_{2}^{\prime}([\mathbf{Y}])=\left[\xi_{*}\left(-\widetilde{Y}_{\varpi}\right)\right] \otimes\{\varpi\}+\left[\xi_{*}\left(-\widetilde{Y}_{\varsigma}\right)\right] \otimes\{\varsigma\}$, as was to be shown.

5.2.2. Proof of Part 2 of Theorem 5.1. Let us begin with two simple lemmas.

LeMma 5.6. 1. The natural projections induce isomorphisms

$$
H^{1}\left(\mathbf{Q}_{p}, T^{-}\right) / \varpi \cong H^{1}\left(\mathbf{Q}_{p}, T^{-} / \varpi\right) ; \quad H^{1}\left(\mathbf{Q}_{p}, T^{-}\right) / \varsigma \cong H^{1}\left(\mathbf{Q}_{p}, T^{-} / \varsigma\right) .
$$

2. $\xi_{*}$ induces an isomorphism $H^{1}\left(\mathbf{Q}_{p}, T^{-}\right) \otimes_{\mathscr{R}} \mathscr{R} / \mathscr{P} \cong H^{1}\left(\mathbf{Q}_{p}, \mathbf{Q}_{p}\right)$.

Proof. 1. We prove the first isomorphism, the other being similar. Since $\left(T^{-} / \varpi\right) / \varsigma=T^{-} / \mathscr{P} \cong \mathbf{Q}_{p}$, $H^{2}\left(\mathbf{Q}_{p}, T^{-} / \varpi\right) / \varsigma$ is a submodule of $H^{2}\left(\mathbf{Q}_{p}, \mathbf{Q}_{p}\right)=0$, hence $H^{2}\left(\mathbf{Q}_{p}, T^{-} / \varpi\right)=0$ by Nakayama's Lemma. We have short exact sequences

$$
0 \longrightarrow H^{q}\left(\mathbf{Q}_{p}, T^{-}\right) / \varpi \longrightarrow H^{q}\left(\mathbf{Q}_{p}, T^{-} / \varpi\right) \longrightarrow H^{q+1}\left(\mathbf{Q}_{p}, T^{-}\right)[\varpi] \longrightarrow 0 .
$$

Taking $q=2$ yields $H^{2}\left(\mathbf{Q}_{p}, T^{-}\right) / \varpi=0$, and then $H^{2}\left(\mathbf{Q}_{p}, T^{-}\right)=0$ by another application of Nakayama's Lemma. Taking now $q=1$ in the exact sequence above, one finds $H^{1}\left(\mathbf{Q}_{p}, T^{-}\right) / \varpi \cong H^{1}\left(\mathbf{Q}_{p}, T^{-} / \varpi\right)$.

2. By an argument similar to that proving Part 1, the vanishing of $H^{2}\left(\mathbf{Q}_{p}, \mathbf{Q}_{p}\right)$ implies that $H^{1}\left(\mathbf{Q}_{p}, T^{-} / \varpi\right) / \varsigma$ is isomorphic to $H^{1}\left(\mathbf{Q}_{p}, \mathbf{Q}_{p}\right)$. Together with Part 1 this concludes the proof.

Taking $\mathscr{S}=\mathbf{Q}_{p}$ and $M=V_{p}(A)$ in (33) (so that $M^{-}=\mathbf{Q}_{p}$ ), one can extract from the long exact sequence a morphism $\jmath: \operatorname{Hom}_{\text {cont }}\left(\mathbf{Q}_{p}^{*}, \mathbf{Q}_{p}\right)=H^{1}\left(\mathbf{Q}_{p}, \mathbf{Q}_{p}\right) \rightarrow \widetilde{H}_{f}^{2}\left(V_{p}(A)\right)$. We recall also the morphism $\wp^{+}: \widetilde{H}_{f}^{1}\left(V_{p}(A)\right) \rightarrow$ $H^{1}\left(\mathbf{Q}_{p}, \mathbf{Q}_{p}(1)\right)=\mathbf{Q}_{p}^{*} \widehat{\otimes} \mathbf{Q}_{p}$ introduced in Section 4.2.

Lemma 5.7. For every $\boldsymbol{x} \in \widetilde{H}_{f}^{1}\left(V_{p}(A)\right)$ and every $\kappa \in H^{1}\left(\mathbf{Q}_{p}, \mathbf{Q}_{p}\right)$ :

$$
\langle\jmath(\kappa), \boldsymbol{x}\rangle_{\mathrm{Nek}}=-\kappa\left(\wp^{+}(\boldsymbol{x})\right) .
$$

Proof. Let $\hat{\kappa} \in C_{\text {cont }}^{1}\left(\mathbf{Q}_{p}, V_{p}(A)\right)$ be a 1-cochain lifting $\kappa$ under $p_{*}^{-}: C_{\text {cont }}^{\bullet}\left(\mathbf{Q}_{p}, V_{p}(A)\right) \rightarrow C_{\text {cont }}^{\bullet}\left(\mathbf{Q}_{p}, \mathbf{Q}_{p}\right)$ and let $d \hat{\kappa}=i^{+}\left(c(\kappa)^{+}\right)$, for a 2-cocycle $c(\kappa)^{+} \in C_{\text {cont }}^{2}\left(\mathbf{Q}_{p}, \mathbf{Q}_{p}(1)\right)$. By construction

$$
\jmath(\kappa)=\left[\left(0, c(\kappa)^{+}, \hat{\kappa}\right)\right] \in \widetilde{H}_{f}^{2}\left(V_{p}(A)\right) .
$$

Let $\left(x, x^{+}, y\right) \in \widetilde{C}_{f}^{1}\left(V_{p}(A)\right)$ be a 1-cocycle representing $\boldsymbol{x}$, so $\wp^{+}(\boldsymbol{x})$ is represented by $x^{+} \in C_{\text {cont }}^{1}\left(\mathbf{Q}_{p}, \mathbf{Q}_{p}(1)\right)$. The definition of $\langle-,-\rangle_{\mathrm{Nek}}$ in [Nek06, Section 6.3] yields

$$
\langle\jmath(\kappa), \boldsymbol{x}\rangle_{\mathrm{Nek}}=\operatorname{inv}_{p}\left(\left[\hat{\kappa} \cup_{W} i^{+}\left(x^{+}\right)\right]\right)=\operatorname{inv}_{p}\left(\kappa \cup \wp^{+}(\boldsymbol{x})\right)=-\kappa\left(\wp^{+}(\boldsymbol{x})\right) .
$$

Here $\cup_{W}: C_{\text {cont }}^{\bullet}\left(\mathbf{Q}_{p}, V_{p}(A)\right) \otimes_{\mathbf{Q}_{p}} C_{\text {cont }}^{\bullet}\left(\mathbf{Q}_{p}, V_{p}(A)\right) \rightarrow C_{\text {cont }}^{\bullet}\left(\mathbf{Q}_{p}, \mathbf{Q}_{p}(1)\right)$ is the cup-product induced by the Weil pairing $W$, and $\operatorname{inv}_{p}: H^{2}\left(\mathbf{Q}_{p}, \mathbf{Q}_{p}(1)\right) \cong \mathbf{Q}_{p}$ is the invariant map. The second equality follows from Remark 4.1, while the last equality is a consequence of local class field theory [Ser67]. 
We are now ready to begin the actual proof of Part 2 of Theorem 5.1. Let $\mathfrak{Z}=\left(\mathfrak{Z}_{n}\right) \in H_{\mathrm{Iw}}^{1}\left(\mathbf{Q}_{\infty}, \mathbb{T}\right)$, let $\mathfrak{z}:=\mathfrak{Z}_{0, \psi}$ and assume that $\mathfrak{z} \in H_{f}^{1}\left(\mathbf{Q}, V_{p}(A)\right)$. As in Section 5.1, Shapiro's Lemma gives a natural isomorphism

$$
H^{1}(\mathfrak{G}, T) \cong H_{\mathrm{Iw}}^{1}\left(\mathbf{Q}_{\infty}, \mathbb{T}\right) \otimes_{R} \mathscr{R} .
$$

Write again $\mathfrak{Z} \in H^{1}(\mathfrak{G}, T)$ for the class corresponding to $\mathfrak{Z} \otimes 1 \in H_{\mathrm{Iw}}^{1}\left(\mathbf{Q}_{\infty}, \mathbb{T}\right) \otimes \bar{R} \mathscr{R}$ under this isomorphism, which satisfies $\mathfrak{z}=\xi_{*}(\mathfrak{Z})$. Choose a 1 -cocycle $Z \in C_{\text {cont }}^{1}(\mathfrak{G}, T)$ representing $\mathfrak{Z}$, and a 1 -cochain

$$
\widetilde{Z}=(Z, \dagger, \ddagger) \in \widetilde{C}_{f}^{1}(T) \quad \text { such that } \quad\left[\xi_{*}(\widetilde{Z})\right]=\mathfrak{z} \in \widetilde{H}_{f}^{1}\left(V_{p}(A)\right) .
$$

(The shape of $\dagger \in C_{\text {cont }}^{1}\left(\mathbf{Q}_{p}, T^{+}\right)$and $\ddagger \in \bigoplus_{l \mid N p} C_{\text {cont }}^{0}\left(\mathbf{Q}_{l}, T\right)$ will not be relevant, and we use (36) to identify $H_{f}^{1}\left(\mathbf{Q}, V_{p}(A)\right)$ with a submodule of $\widetilde{H}_{f}^{1}\left(V_{p}(A)\right)$.) As $\xi_{*}(\widetilde{d}(\widetilde{Z}))=0$, there exist $\widetilde{Y}_{\varpi}, \widetilde{Y}_{\varsigma} \in \widetilde{C}_{f}^{2}(T)$ such that

$$
\widetilde{d}(\widetilde{Z})=\varpi \cdot \widetilde{Y}_{\varpi}+\varsigma \cdot \widetilde{Y}_{\varsigma} .
$$

Write $\widetilde{y}_{\varpi}:=\xi_{*}\left(\widetilde{Y}_{\varpi}\right)$ and $\widetilde{y}_{\varsigma}:=\xi_{*}\left(\widetilde{Y}_{\varsigma}\right)$. Lemma 5.5 yields

$$
-\widetilde{\beta}_{p}(\mathfrak{z})=\left[\widetilde{y}_{\varpi}\right] \otimes\{\varpi\}+\left[\widetilde{y}_{\varsigma}\right] \otimes\{\varsigma\} \in \widetilde{H}_{f}^{2}\left(V_{p}(A)\right) \otimes \mathbf{Q}_{p} \mathscr{P} / \mathscr{P}^{2} .
$$

For $? \in\{\varpi, \varsigma\}$, write $\widetilde{Y}_{?}=\left(Y_{?}, Y_{?}^{+}, \hat{K}_{?}+\hat{L}_{?}\right)$, where

$$
Y_{?} \in C_{\text {cont }}^{2}(\mathfrak{G}, T) ; \quad Y_{?}^{+} \in C_{\text {cont }}^{2}\left(\mathbf{Q}_{p}, T^{+}\right) ; \quad \hat{K}_{?} \in C_{\text {cont }}^{1}\left(\mathbf{Q}_{p}, T\right) ; \quad \hat{L} \in \bigoplus_{l \mid N} C_{\text {cont }}^{1}\left(\mathbf{Q}_{l}, T\right) .
$$

Since $d(Z)=0,(48)$ gives $\varpi \cdot Y_{\varpi}=-\varsigma \cdot Y_{\varsigma}$ and this implies $\xi_{*}\left(Y_{?}\right)=0$, as $T$ and $T^{+}$are free $\mathscr{R}$-modules. Define

$$
y_{?}^{+}:=\xi_{*}\left(Y_{?}^{+}\right) \in C_{\text {cont }}^{2}\left(\mathbf{Q}_{p}, \mathbf{Q}_{p}(1)\right) ; \quad \hat{\kappa}_{?}:=\xi_{*}\left(\hat{K}_{?}\right) \in C_{\text {cont }}^{1}\left(\mathbf{Q}_{p}, V_{p}(A)\right) .
$$

Since $\mathbf{R} \Gamma_{\text {cont }}\left(\mathbf{Q}_{l}, V_{p}(A)\right) \cong 0$ for every prime $l \neq p$ one deduces

$$
\left[\widetilde{y}_{?}\right]=\left[\left(0, y_{?}^{+}, \hat{\kappa}_{?}\right)\right]=\jmath\left(\kappa_{?}\right) ; \quad \kappa_{?}:=p_{*}^{-}\left(\hat{\kappa}_{?}\right) \in H^{1}\left(\mathbf{Q}_{p}, \mathbf{Q}_{p}\right)
$$

(see equation (47)). Lemma 5.7 and (49) then give: for every $\boldsymbol{x} \in \widetilde{H}_{f}^{1}\left(V_{p}(A)\right)$

$$
\left\langle\langle\mathfrak{z}, \boldsymbol{x}\rangle_{p}=\langle-,-\rangle_{\mathrm{Nek}} \otimes \overline{\mathrm{M}}\left(\widetilde{\beta}_{p}(\mathfrak{z}) \otimes \boldsymbol{x}\right)=\log _{p}(\varpi) \cdot \kappa_{\varpi}\left(\wp^{+}(\boldsymbol{x})\right) \cdot\{k-2\}+\log _{p}(\varsigma) \cdot \kappa_{\varsigma}\left(\wp^{+}(\boldsymbol{x})\right) \cdot\{s-1\} .\right.
$$

Lemma 5.8. The class $\operatorname{res}_{p}(\mathfrak{Z})$ belongs to $H^{1}\left(\mathbf{Q}_{p}, T\right)^{\circ}$ and we have

$$
\begin{gathered}
\log _{p}(\varsigma) \cdot \kappa_{\varsigma}\left(p^{-1}\right)=-\operatorname{Der}_{\text {cyc }}\left(\operatorname{res}_{p}(\mathfrak{Z})\right) ; \quad \log _{p}(\varpi) \cdot \kappa_{\varpi}(e(1))=-\operatorname{Der}_{\mathrm{wt}}\left(\operatorname{res}_{p}(\mathfrak{Z})\right) ; \\
\log _{p}(\varpi) \cdot \kappa_{\varpi}\left(p^{-1}\right)-\frac{1}{2} \log _{p}(\varsigma) \cdot \mathscr{L}_{p}(A) \cdot \kappa_{\varsigma}(e(1))=-\operatorname{Der}_{\dagger}\left(\operatorname{res}_{p}(\mathfrak{Z})\right) .
\end{gathered}
$$

Proof. Since $\mathfrak{z}$ is a Selmer class, $p^{-}\left(\operatorname{res}_{p}(\mathfrak{Z})\right)$ is in the kernel of $\xi_{*}: H^{1}\left(\mathbf{Q}_{p}, T^{-}\right) \rightarrow H^{1}\left(\mathbf{Q}_{p}, \mathbf{Q}_{p}\right)(\operatorname{see}(34))$. Lemma 5.6(2) then implies that $\operatorname{res}_{p}(\mathfrak{Z}) \in H^{1}\left(\mathbf{Q}_{p}, T\right)^{\circ}$.

Write $K_{?}:=p_{*}^{-}\left(\hat{K}_{?}\right)$, so that $\xi_{*}\left(K_{?}\right)=\kappa_{\text {? }}$. By equation $(48)$

$$
-p_{*}^{-}\left(\operatorname{res}_{p}(Z)\right) \approx \varpi \cdot K_{\varpi}+\varsigma \cdot K_{\varsigma},
$$

where $\approx$ denotes equality up to coboundaries. In particular the sum in the R.H.S. is a 1-cocycle in $C_{\text {cont }}^{1}\left(\mathbf{Q}_{p}, T^{-}\right)$. Then $\varpi \cdot\left(K_{\varpi} \bmod \varsigma\right) \in C_{\text {cont }}^{1}\left(\mathbf{Q}_{p}, T^{-} / \varsigma\right)$ is a 1 -cocycle, so $\left(K_{\varpi} \bmod \varsigma\right)$ is a 1 -cocycle, as $T^{-} / \varsigma$ is free over $\mathscr{R} / \varsigma$. Similarly, $\left(K_{\varsigma} \bmod \varpi\right) \in C_{\text {cont }}^{1}\left(\mathbf{Q}_{p}, T^{-} / \varpi\right)$ is a 1-cocycle. Lemma 5.6 then implies the existence of 1-cocycles $A_{\varpi}, A_{\varsigma} \in C_{\text {cont }}^{1}\left(\mathbf{Q}_{p}, T^{-}\right)$and 1-cochains $B_{\varpi}, B_{\varsigma} \in C_{\text {cont }}^{1}\left(\mathbf{Q}_{p}, T^{-}\right)$such that

$$
A_{\varpi} \approx K_{\varpi}+\varsigma \cdot B_{\varpi} ; \quad A_{\varsigma} \approx K_{\varsigma}+\varpi \cdot B_{\varsigma} .
$$

Note that $\varpi \varsigma \cdot\left(B_{\varpi}+B_{\varsigma}\right) \in C_{\text {cont }}^{1}\left(\mathbf{Q}_{p}, T^{-}\right)$is a 1-cocycle; using again the fact that $T^{-}$is $\mathscr{R}$-free, this implies that $B_{\varpi}+B_{\varsigma}$ itself is a 1-cocycle. We then deduce the congruence

$$
-p^{-}\left(\operatorname{res}_{p}(\mathfrak{Z})\right)=\left[\varpi \cdot K_{\varpi}+\varsigma \cdot K_{\varsigma}\right] \equiv \varpi \cdot\left[A_{\varpi}\right]+\varsigma \cdot\left[A_{\varsigma}\right] \quad\left(\bmod \mathscr{P}^{2} \cdot H^{1}\left(\mathbf{Q}_{p}, T^{-}\right)\right) .
$$

Since $\kappa_{\varpi}=\xi_{*}\left(\left[A_{\varpi}\right]\right)$ and $\kappa_{\varsigma}=\xi_{*}\left(\left[A_{\varsigma}\right]\right)$, the lemma follows from the definition of the derivatives of $\operatorname{res}_{p}(\mathfrak{Z})$.

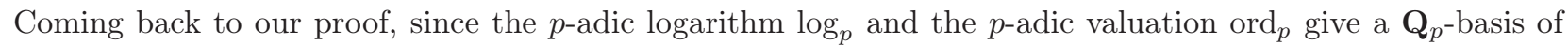
$\operatorname{Hom}_{\text {cont }}\left(\mathbf{Q}_{p}^{*}, \mathbf{Q}_{p}\right)$, Lemma 5.8 allows us to write

$(51)-\log _{p}(\varsigma) \cdot \kappa_{\varsigma}:=a(\varsigma) \cdot \log _{p}-\operatorname{Der}_{\mathrm{cyc}}\left(\operatorname{res}_{p}(\mathfrak{Z})\right) \cdot \operatorname{ord}_{p} ; \quad-\log _{p}(\varpi) \cdot \kappa_{\varpi}=\operatorname{Der}_{\mathrm{wt}}\left(\operatorname{res}_{p}(\mathfrak{Z})\right) \cdot \log _{p}+b(\varpi) \cdot \operatorname{ord}_{p}$, for (unique) constants $a(\varsigma), b(\varpi) \in \mathbf{Q}_{p}$ which satisfy

$$
b(\varpi)+\frac{1}{2} \mathscr{L}_{p}(A) \cdot a(\varsigma)=-\operatorname{Der}_{\dagger}\left(\operatorname{res}_{p}(\mathfrak{Z})\right) .
$$


Since $\wp^{+}(\mathfrak{z}) \in \mathbf{Z}_{p}^{*} \widehat{\otimes} \mathbf{Q}_{p}$ by (35) and $\wp^{+}\left(q_{A}\right)=q_{A} \widehat{\otimes} 1$ (cf. (36)), equations (50) and (51) yield

$$
-\left\langle\langle\mathfrak{z}, \mathfrak{z}\rangle_{p}=a(\varsigma) \cdot \log _{A}\left(\operatorname{res}_{p}(\mathfrak{z})\right) \cdot\{s-1\}+\operatorname{Der}_{\mathrm{wt}}\left(\operatorname{res}_{p}(\mathfrak{Z})\right) \cdot \log _{A}\left(\operatorname{res}_{p}(\mathfrak{z})\right) \cdot\{k-2\},\right.
$$

and

$$
\begin{aligned}
-\left\langle\left\langle\mathfrak{z}, q_{A}\right\rangle_{p}=(a(\varsigma) \cdot\right. & \left.\left.\log _{p}\left(q_{A}\right)-\operatorname{Der}_{\mathrm{cyc}}\left(\operatorname{res}_{p}(\mathfrak{Z})\right) \cdot \operatorname{ord}_{p}\left(q_{A}\right)\right)\right) \cdot\{s-1\} \\
& \left.+\left(\operatorname{Der}_{\mathrm{wt}}\left(\operatorname{res}_{p}(\mathfrak{Z})\right) \cdot \log _{p}\left(q_{A}\right)+b(\varpi) \cdot \operatorname{ord}_{p}\left(q_{A}\right)\right)\right) \cdot\{k-2\}
\end{aligned}
$$

(where we have used the formula $\log _{p} \circ \wp^{+}(\mathfrak{z})=\log _{A}\left(\operatorname{res}_{p}(\mathfrak{z})\right.$ ), which follows immediately retracing the definitions of $\log _{A}$ and $\wp^{+}$). Moreover, the exceptional zero formulae displayed in Theorem 4.2(2) give the identities

$$
\left.\left\langle q_{A}, q_{A}\right\rangle\right\rangle_{p}=\log _{p}\left(q_{A}\right) \cdot\{s-k / 2\} ; \quad\left\langle q_{A}, \mathfrak{z}\right\rangle_{p}=\log _{A}\left(\operatorname{res}_{p}(\mathfrak{z})\right) \cdot\{s-1\} .
$$

Using equations (52), (53), (54) and (55) and writing for simplicity $\operatorname{Der}$ ? $(\mathfrak{Z}):=\operatorname{Der}_{\text {? }}\left(\operatorname{res}_{p}(\mathfrak{Z})\right)$, we compute

$$
\frac{-\widetilde{h}_{p}(\mathfrak{z})}{\operatorname{ord}_{p}\left(q_{A}\right)}=\log _{A}\left(\operatorname{res}_{p}(\mathfrak{z})\right) \times\left(\operatorname{Der}_{\mathrm{cyc}}(\mathfrak{Z}) \cdot\{s-1\}^{2}+\operatorname{Der}_{\dagger}(\mathfrak{Z}) \cdot\{s-1\}\{k-2\}-\frac{1}{2} \mathscr{L}_{p}(A) \cdot \operatorname{Der}_{\mathrm{wt}}(\mathfrak{Z}) \cdot\{k-2\}^{2}\right)
$$

in $\mathscr{J}^{2} / \mathscr{J}^{3}$. Part 2 of Theorem 5.1 follows by combining the last equation with Corollary 5.4.

\section{Proofs of the main results}

In this section we prove the results stated in the introduction.

6.1. Proof of Theorem A. As in Section 1.3, let $L_{p}^{\mathrm{cc}}\left(f_{\infty}, k\right):=L_{p}\left(f_{\infty}, k, k / 2\right) \in \mathscr{A}_{U}$ and let

$$
\widetilde{h}_{p}^{\mathrm{cc}}: H_{f}^{1}\left(\mathbf{Q}, V_{p}(A)\right) \rightarrow \mathbf{Q}_{p}
$$

be the composition of $\widetilde{h}_{p}$ with the morphism $\mathscr{J}^{2} / \mathscr{J}^{3} \rightarrow \mathbf{Q}_{p}$ sending $\alpha(k, s) \in \mathscr{J}^{2}$ to $\frac{d^{2}}{d k^{2}} \alpha(k, k / 2)_{k=2}$. By the functional equation for $\left\langle\langle-,-\rangle_{p}(k, s)\right.$ stated in Theorem $4.2(3),\langle-,-\rangle_{p}(k, k / 2)$ is a skew-symmetric pairing on $\widetilde{H}_{f}^{1}\left(\mathbf{Q}, V_{p}(A)\right)$. Together with Theorem 4.2(2) this gives

$$
\widetilde{h}_{p}^{\mathrm{cc}}(x)=\left.\frac{d^{2}}{d k^{2}} \operatorname{det}\left(\begin{array}{cc}
0 & \frac{1}{2} \log _{A}\left(\operatorname{res}_{p}(x)\right) \cdot(k-2) \\
-\frac{1}{2} \log _{A}\left(\operatorname{res}_{p}(x)\right) \cdot(k-2) & 0
\end{array}\right)\right|_{k=2}=\frac{1}{2} \log _{A}^{2}\left(\operatorname{res}_{p}(x)\right),
$$

for every Selmer class $x \in H_{f}^{1}\left(\mathbf{Q}, V_{p}(A)\right)$.

Assume that $L(A / \mathbf{Q}, 1)=0$, i.e. that $\zeta^{\mathrm{BK}}$ is a Selmer class by Kato's reciprocity (2). Combining the Bertolini-Darmon exceptional zero formula of Theorem 2.1, Theorem 5.3 and equation (56), one obtains the identity

$$
\log _{A}\left(\operatorname{res}_{p}\left(\zeta^{\mathrm{BK}}\right)\right) \cdot 2 \ell \cdot \log _{A}^{2}(\mathbf{P})=\frac{-1}{\operatorname{ord}_{p}\left(q_{A}\right)}\left(1-\frac{1}{p}\right)^{-1} \cdot \log _{A}^{2}\left(\operatorname{res}_{p}\left(\zeta^{\mathrm{BK}}\right)\right),
$$

for a non-zero rational number $\ell \in \mathbf{Q}^{*}$ and a rational point $\mathbf{P} \in A(\mathbf{Q}) \otimes \mathbf{Q}$. Moreover, $\mathbf{P} \neq 0$ precisely if $L(A / \mathbf{Q}, s)$ has a simple zero at $s=1$. In order to conclude the proof of Theorem A, we need the following lemma. For every $\mathfrak{Z} \in H^{1}(\mathfrak{G}, T)$, write $\mathcal{L}_{\mathbb{T}}^{\mathrm{cc}}\left(\operatorname{res}_{p}(\mathfrak{Z}), k\right)$ for the restriction of $\mathcal{L}_{\mathbb{T}}\left(\operatorname{res}_{p}(\mathfrak{Z}), k, s\right)$ to the central critical line $s=k / 2$, and let $\xi_{*}: H^{1}(\mathfrak{G}, T) \rightarrow H^{1}\left(\mathfrak{G}, V_{p}(A)\right)$ be the morphism induced by (38).

Lemma 6.1. Let $\mathfrak{Z} \in H^{1}(\mathfrak{G}, T)$ be such that $\xi_{*}(\mathfrak{Z}) \in H_{f}^{1}\left(\mathbf{Q}, V_{p}(A)\right)$. The following statements are equivalent:

(a) $\xi_{*}(\mathfrak{Z})$ is in the kernel of the restriction map $\operatorname{res}_{p}: H_{f}^{1}\left(\mathbf{Q}, V_{p}(A)\right) \rightarrow A\left(\mathbf{Q}_{p}\right) \widehat{\otimes} \mathbf{Q}_{p}$.

(b) $\mathcal{L}_{\mathbb{T}}^{\mathrm{cc}}\left(\operatorname{res}_{p}(\mathfrak{Z}), k\right)$ vanishes to order (strictly) greater than 2 at $k=2$.

Proof. Write $\mathfrak{z}:=\xi_{*}(\mathfrak{Z})$. Theorem 5.1(2) and equation (56) yield

$$
\log _{A}\left(\operatorname{res}_{p}(\mathfrak{z})\right) \cdot \frac{d^{2}}{d k^{2}} \mathcal{L}_{\mathbb{T}}^{\mathrm{cc}}\left(\operatorname{res}_{p}(\mathfrak{Z}), k\right)_{k=2} \doteq \log _{A}^{2}\left(\operatorname{res}_{p}(\mathfrak{z})\right),
$$

where $\doteq$ denotes equality up to a non-zero rational factor. Since $\log _{A}: A\left(\mathbf{Q}_{p}\right) \widehat{\otimes} \mathbf{Q}_{p} \cong \mathbf{Q}_{p}$ is an isomorphism, this shows that $(b)$ implies $(a)$.

Assume now that $(a)$ holds. Since $0=\operatorname{res}_{p}\left(\xi_{*}(\mathfrak{Z})\right)=\xi_{*}\left(\operatorname{res}_{p}(\mathfrak{Z})\right)$, one can write $\operatorname{res}_{p}(\mathfrak{Z})=\varsigma \cdot \mathfrak{Z}_{\varsigma}+\varpi \cdot \mathfrak{Z}_{\varpi}$, for classes $\mathfrak{Z}_{\varsigma}, \mathfrak{Z}_{\varpi} \in H^{1}\left(\mathbf{Q}_{p}, T\right)$. (Indeed, as $H^{2}\left(\mathbf{Q}_{p}, V_{p}(A)\right)=0$, an argument similar to the one appearing in the proof of Lemma 5.6(2) proves that $H^{1}\left(\mathbf{Q}_{p}, T\right) \otimes \mathscr{R}, \xi \mathbf{Q}_{p} \cong H^{1}\left(\mathbf{Q}_{p}, V_{p}(A)\right)$.) By Theorem 3.1(2)

$$
\mathcal{L}_{\mathbb{T}}\left(\operatorname{res}_{p}(\mathfrak{Z}), k, s\right) \equiv \mathscr{L}_{p}(A) \cdot\left(\exp _{A}^{*}\left(\mathfrak{z}_{\varsigma}\right) \cdot(s-1)+\exp _{A}^{*}\left(\mathfrak{z}_{\varpi}\right) \cdot(k-2)\right) \cdot(s-k / 2)\left(\bmod \mathscr{J}^{3}\right),
$$


where $\mathfrak{z}_{\varpi}:=\log _{p}(\varpi) \cdot \xi_{*}\left(\mathfrak{Z}_{\varpi}\right), \mathfrak{z}_{\varsigma}:=\log _{p}(\varsigma) \cdot \xi_{*}\left(\mathfrak{Z}_{\varsigma}\right) \in H^{1}\left(\mathbf{Q}_{p}, V_{p}(A)\right)$. This shows that $(a)$ implies $(b)$, thus concluding the proof of the lemma.

Coming back to our proof, the preceding lemma, applied to $\mathfrak{Z}=\mathfrak{Z}_{\infty}^{\mathrm{BK}}$, tells us that $\operatorname{res}_{p}\left(\zeta^{\mathrm{BK}}\right)=0$ (or equivalently $\left.\log _{A}\left(\operatorname{res}_{p}\left(\zeta^{\mathrm{BK}}\right)\right)=0\right)$ if and only if $L_{p}^{\mathrm{cc}}\left(f_{\infty}, k\right)$ vanishes to order greater than 2 at $k=2$. In addition, Theorem 2.1 tells us that the latter condition is equivalent to $\mathbf{P}=0$. To sum up: $\operatorname{res}_{p}\left(\zeta^{\mathrm{BK}}\right)$ is non-zero if and only if $\mathbf{P}$ is non-zero. Defining $\ell_{1}:=-2 \ell \cdot \operatorname{ord}_{p}\left(q_{A}\right) \cdot\left(1-p^{-1}\right) \in \mathbf{Q}^{*}$, equation (57) then gives

$$
\log _{A}\left(\operatorname{res}_{p}\left(\zeta^{\mathrm{BK}}\right)\right)=\ell_{1} \cdot \log _{A}^{2}(\mathbf{P}),
$$

concluding the proof of Theorem A.

6.2. Proof of Theorem B. Write $r_{\mathrm{an}}:=\operatorname{ord}_{s=1} L(A / \mathbf{Q}, s)$. That $r_{\text {an }} \leq 1$ implies $\zeta^{\mathrm{BK}} \neq 0$ follows from Kato's reciprocity law (2) (if $r_{\mathrm{an}}=0$ ) and Theorem A (if $r_{\mathrm{an}}=1$ ).

Conversely, assume that $\zeta^{\mathrm{BK}}$ is non-zero. The method of Kolyvagin, applied to the Euler system constructed by Kato in [Kat04], then tells us that the strict Selmer group

$$
\left\{x \in H^{1}\left(\mathfrak{G}, V_{p}(A)\right): \operatorname{res}_{p}(x)=0\right\} \subset H_{f}^{1}\left(\mathbf{Q}, V_{p}(A)\right)
$$

is trivial. For a proof of this result, see Theorem 2.3 and Chapter III, Section 5 of [Rub00]. (Note that $A$ does not have complex multiplication, since $\operatorname{ord}_{p}\left(j_{A}\right)=-\operatorname{ord}_{p}\left(q_{A}\right)<0$ [Sil94, Theorem 6.1]. This implies that the hypotheses of [Rub00, Theorem 2.3] are satisfied.) Then the restriction $\operatorname{res}_{p}\left(\zeta^{\mathrm{BK}}\right)$ is non-zero. Using again Theorem A (resp., equation (2)), one deduces that $r_{\mathrm{an}}=1$ (resp., $\left.r_{\mathrm{an}}=0\right)$ if $\zeta^{\mathrm{BK}}$ is (resp., is not) a Selmer class.

6.3. An interlude. In the proofs of Theorems C-E, we need the following lemma.

Lemma 6.2. Assume that (Loc) holds and that $\operatorname{ord}_{s=1} L_{p}(A / \mathbf{Q}, s)=2$. Then $\zeta^{\mathrm{BK}} \neq 0$.

Proof. We have short exact sequences of $\mathbf{Q}_{p}$-modules (easily deduced from Shapiro's Lemma):

$$
0 \rightarrow H_{\mathrm{IW}}^{q}\left(\mathbf{Q}_{\infty}, V_{p}(A)\right) / \varsigma \rightarrow H^{q}\left(\mathfrak{G}, V_{p}(A)\right) \rightarrow H_{\mathrm{Iw}}^{q+1}\left(\mathbf{Q}_{\infty}, V_{p}(A)\right)[\varsigma] \rightarrow 0
$$

where $H_{\mathrm{Iw}}^{q}\left(\mathbf{Q}_{\infty}, V_{p}(A)\right):=H_{\mathrm{Iw}}^{q}\left(\mathbf{Q}_{\infty}, T_{p}(A)\right) \otimes_{\mathbf{z}_{p}} \mathbf{Q}_{p}$. Since $H^{0}\left(\mathfrak{G}, V_{p}(A)\right)=0, H_{\mathrm{Iw}}^{1}\left(\mathbf{Q}_{\infty}, V_{p}(A)\right)$ has no non-trivial $\varsigma$-torsion. Moreover a theorem of Rohrlich [Roh84] states that $L_{p}(A / \mathbf{Q}) \neq 0$, so in particular $\zeta_{\infty}^{\mathrm{BK}} \neq 0$ by $(1)$. There exist then a unique class $z_{\infty}^{\mathrm{BK}}=\left(z_{n}^{\mathrm{BK}}\right) \in H_{\mathrm{Iw}}^{1}\left(\mathbf{Q}_{\infty}, V_{p}(A)\right)$ and a unique integer $\rho=\rho_{\mathrm{BK}} \geq 0$ such that

$$
\zeta_{\infty}^{\mathrm{BK}}=\varsigma^{\rho} \cdot z_{\infty}^{\mathrm{BK}} ; \quad 0 \neq z_{0}^{\mathrm{BK}} \in H^{1}\left(\mathfrak{G}, V_{p}(A)\right) .
$$

By Poitou-Tate duality and hypothesis $($ Loc $)$ one has $H_{f}^{1}\left(\mathbf{Q}, V_{p}(A)\right)=H^{1}\left(\mathfrak{G}, V_{p}(A)\right)$ (see Lemme 2.3.9 of [PR93]). In particular $z_{0}^{\mathrm{BK}} \in H_{f}^{1}\left(\mathbf{Q}, V_{p}(A)\right)$, so that

$$
\mathcal{L}_{A}\left(\operatorname{res}_{p}\left(z_{\infty}^{\mathrm{BK}}\right)\right) \in \varsigma^{2} \cdot \Lambda_{\mathrm{cyc}}
$$

by Corollary 3.7. This yields

$$
L_{p}(A / \mathbf{Q})=\mathcal{L}_{A}\left(\operatorname{res}_{p}\left(\zeta_{\infty}^{\mathrm{BK}}\right)\right) \in \varsigma^{\rho+2} \cdot \Lambda_{\mathrm{cyc}},
$$

i.e. $\operatorname{ord}_{s=1} L_{p}(A / \mathbf{Q}, s) \geq \rho+2$. Our assumption then forces $\rho=0$ and $\zeta^{\mathrm{BK}}=z_{0}^{\mathrm{BK}} \neq 0$, as was to be shown.

6.4. Proof of Theorem C. Assume that $\operatorname{sign}(A / \mathbf{Q})=-1$ and that hypothesis (Loc) is satisfied. Given $x \in H_{f}^{1}\left(\mathbf{Q}, V_{p}(A)\right)$, write for simplicity $\log _{A}(x)=\log _{A}\left(\operatorname{res}_{p}(x)\right)$.

6.4.1. Step I. Assume that $\mathbf{P}$ is non-zero, i.e. that $\operatorname{ord}_{s=1} L(A / \mathbf{Q}, s)=1$. Thanks to the work of GrossZagier [GZ86] and Kolyvagin $[\mathbf{K o l 9 0}], A(\mathbf{Q})$ has rank one and $A(\mathbf{Q}) \otimes \mathbf{Q}_{p} \cong H_{f}^{1}\left(\mathbf{Q}, V_{p}(A)\right)$. One can then write $\zeta^{\mathrm{BK}}=\lambda \cdot \mathbf{P}$, where $\lambda=\log _{A}\left(\zeta^{\mathrm{BK}}\right) / \log _{A}(\mathbf{P})$, so that $\widetilde{h}_{p}\left(\zeta^{\mathrm{BK}}\right)=\lambda^{2} \cdot \widetilde{h}_{p}(\mathbf{P})$. Setting $\ell_{2}:=2 \ell$, Theorem A and Theorem 5.3 combine to give the identity

$$
L_{p}\left(f_{\infty}, k, s\right) \bmod \mathscr{J}^{3}=\ell_{2} \cdot \widetilde{h}_{p}(\mathbf{P}) .
$$

6.4.2. Step II. Assume that $\mathbf{P}=0$. We claim that

$$
L_{p}\left(f_{\infty}, k, s\right) \in \mathscr{J}^{3} .
$$

Indeed $\operatorname{ord}_{s=1} L(A / \mathbf{Q}, s)>1$ under our assumptions, so that $\zeta^{\mathrm{BK}}=0$ by Theorem B. Lemma 6.2 then yields

$$
\left.\frac{\partial^{2}}{\partial s^{2}} L_{p}\left(f_{\infty}, k, s\right)\right|_{(k, s)=(2,1)}=\frac{d^{2}}{d s^{2}} L_{p}(A / \mathbf{Q}, s)_{s=1}=0 \text {. }
$$

Moreover, by the functional equation (22) and Theorem 2.1

$$
\left.\left(\frac{\partial^{2}}{\partial k^{2}}-\frac{1}{4} \frac{\partial^{2}}{\partial s^{2}}\right) L_{p}\left(f_{\infty}, k, s\right)\right|_{(k, s)=(2,1)}=\frac{d^{2}}{d k^{2}} L_{p}^{\mathrm{cc}}\left(f_{\infty}, k\right)_{k=2}=0 .
$$

Since $L_{p}\left(f_{\infty}, k, s\right) \in \mathscr{J}^{2}$ by Theorem 5.2, the claim (58) follows from the preceding two equations. 
6.4.3. Step III (conclusions). We now prove Theorem C. First of all, $L_{p}\left(f_{\infty}, k, s\right) \in \mathscr{J}^{2}$ by $(2)$ and Theorem 5.2. The $p$-adic Gross-Zagier formula which appears in the statement follows from Steps I and II. Finally, the last assertion in the statement is a direct consequence of Theorem 2.1 and Step II.

6.5. Proof of Theorem D. Assume that (Loc) holds.

If $\operatorname{sign}(A / \mathbf{Q})=+1$, then $\mathbf{P}=0$ and the order of vanishing of $L_{p}(A / \mathbf{Q}, s)$ at $s=1$ is odd by (22). Moreover $\frac{d}{d s} L_{p}(A / \mathbf{Q}, s)_{s=1}=0$, as follows from (2) and Theorem 5.2. Theorem D follows in this case.

Assume now that $\operatorname{sign}(A / \mathbf{Q})=-1$. As above, one easily proves that $\operatorname{ord}_{s=1} L_{p}(A / \mathbf{Q}, s) \geq 2$. Moreover, writing $\widetilde{h}_{p}(\mathbf{P} ; k, s)=\widetilde{h}_{p}(\mathbf{P})$, Theorem 4.2 yields

$$
\left.\widetilde{h}_{p}(\mathbf{P} ; k, s)\right|_{k=2}=\operatorname{det}\left(\begin{array}{cc}
\log _{p}\left(q_{A}\right) & \log _{A}(\mathbf{P}) \\
\log _{A}(\mathbf{P}) & \langle\mathbf{P}, \mathbf{P}\rangle_{p}^{c y c}
\end{array}\right) \cdot\{s-1\}^{2}=\log _{p}\left(q_{A}\right) \cdot\langle\mathbf{P}, \mathbf{P}\rangle_{p}^{\text {Sch }} \cdot\{s-1\}^{2} .
$$

Setting $\ell_{3}:=2 \ell_{2} \cdot \operatorname{ord}_{p}\left(q_{A}\right)^{-1}$ and recalling that $L_{p}(A / \mathbf{Q}, s)=L_{p}\left(f_{\infty}, 2, s\right)$ by equation (17), Theorem D follows by restricting the formula displayed in Theorem $\mathrm{C}$ to the cyclotomic line $k=2$.

6.6. Proof of Theorem E. Assume that $\operatorname{sign}(A / \mathbf{Q})=-1$ and that (Loc) holds.

Writing as above $\widetilde{h}_{p}(\cdot ; k, s)=\widetilde{h}_{p}(\cdot)$, Theorem 4.2 gives

$$
\frac{d^{2}}{d k^{2}} \widetilde{h}_{p}(\mathbf{P} ; k, 1)_{k=2}=2 \operatorname{det}\left(\begin{array}{cc}
-\frac{1}{2} \log _{p}\left(q_{A}\right) & 0 \\
-\log _{A}(\mathbf{P}) & \langle\mathbf{P}, \mathbf{P}\rangle_{p}^{\mathrm{wt}}
\end{array}\right)=-\log _{p}\left(q_{A}\right) \cdot\langle\mathbf{P}, \mathbf{P}\rangle_{p}^{\mathrm{wt}}
$$

On the other hand, by (23) and Theorem 3.18 of [GS93]

$$
\frac{1}{2} \frac{d^{2}}{d k^{2}} L_{p}\left(f_{\infty}, k, 1\right)_{k=2}=\frac{d}{d k}\left(1-a_{p}(k)^{-1}\right)_{k=2} \cdot \frac{d}{d k} L_{p}^{*}\left(f_{\infty}, k\right)_{k=2}=-\frac{1}{2} \mathscr{L}_{p}(A) \cdot \frac{d}{d k} L_{p}^{*}\left(f_{\infty}, k\right)_{k=2} .
$$

Since $\mathscr{L}_{p}(A) \neq 0$ [BSDGP96], Theorem $\mathrm{C}$ and the preceding two equations yield the identity

$$
\frac{d}{d k} L_{p}^{*}\left(f_{\infty}, k\right)_{k=2}=2 \ell_{4} \cdot\langle\mathbf{P}, \mathbf{P}\rangle_{p}^{\mathrm{wt}} ; \quad \ell_{4}:=\ell_{2} \cdot \operatorname{ord}_{p}\left(q_{A}\right)
$$

To conclude the proof, it remains to show that $2\langle\mathbf{P}, \mathbf{P}\rangle_{p}^{\mathrm{wt}}=-\langle\mathbf{P}, \mathbf{P}\rangle_{p}^{\text {cyc }}$. This follows from Theorem 4.2(3).

\section{References}

[BD98] M. Bertolini and H. Darmon. Heegner points, p-adic L-functions and the Cerednik-Drinfeld uniformization. Invent. Math., 131, 1998.

[BD07] M. Bertolini and H. Darmon. Hida families and rational points on elliptic curves. Invent. Math., $168(2), 2007$.

[BD14] M. Bertolini and H. Darmon. Kato's Euler system and rational points on elliptic curves I: A p-adic Beilinson formula. Israel J. Math., 199(1), 2014.

[BDP13] M. Bertolini, H. Darmon, and K. Prasanna. Generalized Heegner cycles and p-adic Rankin L-series. With an appendix by Brian Conrad. Duke Math. J., 162(6), 2013.

[Ber77] D. Bertrand. Transcendence et lois de groupes algébriques. Séminaire Delange-Pisot-Poitou. Théorie de nombres, 18(1), 1976-1977.

[BK90] S. Bloch and K. Kato. L-functions and Tamagawa numbers of motives . In P. Cartier, L. Illusie, N. Katz, and G. Laumon, editors, The Grothendieck Festschrift. Modern Birkhäuser Classics, 1990.

[BSDGP96] K. Barré-Sirieix, G. Diaz, F. Gramain, and G. Philibert. Une Preuve de la Conjecture de Mahler-Manin. Invent. Math., $124,1996$.

[Col79] R. Coleman. Division values in local fields. Invent. Math., 53, 1979.

[EPW06] M. Emerton, R. Pollack, and T. Weston. Variation of Iwasawa invariants in Hida families. Invent. Math., $163,2006$.

[GS93] R. Greenberg and G. Stevens. p-adic L-functions and p-adic periods of modular forms. Invent. Math., 4(111), 1993.

[GV00] R. Greenberg and V. Vatsal. On the Iwasawa invariants of elliptic curves. Invent. Math., 142, 2000.

[GZ86] B. Gross and D. Zagier. Heegner points and derivatives of L-series. Invent. Math., 86(2), 1986.

[Hid86a] H. Hida. Galois representations into $G L_{2}\left(\mathbf{Z}_{p} \llbracket X \rrbracket\right)$ attached to ordinary cusp forms. Invent. Math., 85, 1986.

[Hid86b] H. Hida. Iwasawa modules attached to congruences of cusp forms. Ann. scient. Éc. Norm. Sup., $19,1986$.

[Kat93] K. Kato. Lectures in the approach to Iwasawa theory for Hasse-Weil L-functions via Bdr. In Arithmetic Algebraic Geometry (Trento 1991). Lecture Notes in Math. 1553, New York: Springer-Verlag, 1993.

[Kat04] K. Kato. p-adic Hodge theory and values of zeta functions of modular forms. Astérisque, 295, 2004.

[Kit94] K. Kitagawa. On Standard p-adic L-functions of Families of Elliptic Cusp Forms . In B Mazur and G. Stevens, editors, p-adic monodromy and the Birch and Swinnerton-Dyer conjecture. American Mathematical Society, 1994.

[Kob13] S. Kobayashi. The p-adic Gross-Zagier formula for elliptic curves at supersingular primes. Invent. Math., $191(3), 2013$.

[Kol90] V. A. Kolyvagin. Euler systems. In The Grothendieck Festschrift, Vol. II. Progr. Math., 87, Birkhäuser Boston, Boston, MA, 1990.

[LVZ15] D. Loeffler, O. Venjacob, and S. L. Zerbes. Local epsilon isomorphisms. Kyoto J. Math., 55(1), 2015.

[Mok11] C.P. Mok. Heegner points and p-adic L-functions for elliptic curves over certain totally real fields. Commentarii Mathematici Helvetici, 86, 2011. 
[MT90] B. Mazur and J. Tilouine. Représentations galoisiennes, différentielles de Kähler et "conjectures principales". Publ. Math. de l'I.H.E.S., 71, 1990.

[MTT86] B. Mazur, J. Tate, and J. Teitelbaum. On p-adic analogues of the conjectures of Birch and Swinnerton-Dyer. Invent. Math., 126, 1986 .

[Nek93] J. Nekovar. On p-adic height pairings. In Seminaire de Theorie des Nombres, Paris, 1990-91. Progr. in Math., 108, Birkhäuser Boston, 1993.

[Nek06] J. Nekovar. Selmer complexes. Astérisque, 310, 2006.

[NP00] J. Nekovar and A. Plater. On the parity of ranks of Selmer groups. Asian J. Math., 4(2), 2000.

[Och03] T. Ochiai. A generalization of the Coleman map for Hida deformations. Amer. J. Math., 125(4), 2003.

[Och06] T. Ochiai. On the two-variable Iwasawa main conjecture. Compositio Math., 142, 2006.

[Oht95] M. Ohta. On the p-adic Eichler-Shimura isomorphism for $\Lambda$-adic cusp forms. J. Reine Angew. Math., $463,1995$.

[PR87] B. Perrin-Riou. Points de Heegner et dérivées de fonctions L p-adiques. Invent. Math., 89(3), 1987.

[PR92] B. Perrin-Riou. Thèorie d'Iwasawa et hauteurs p-adiques. Invent. Math, 109, 1992.

[PR93] B. Perrin-Riou. Fonctions L p-adiques d'une courbe elliptique et points rationnels. Ann. Inst. Fourier, Grenoble, 43(4), 1993.

[PR94] B. Perrin-Riou. Théorie d'Iwasawa des représentations p-adiques sur un corps local. With an appendix by Jean-Marc Fontaine. Invent. Math, 115(1), 1994.

[Roh84] D. E. Rohrlich. On L-functions of elliptic curves and cyclotomic towers. Invent. Math., 75(3), 1984.

[Rub94] K. Rubin. Abelian varieties, p-adic heights and derivatives. In G. Frey and J. Ritter, editors, Algebra and Number Theory. de Gruyter, 1994.

[Rub98] K. Rubin. Euler systems and modular elliptic curves. In A. Scholl and R. Taylor, editors, Galois representations in arithmetic algebraic geometry. London Math. Soc. Lect. Notes 254, Cambridge: Cambridge University Press, 1998.

[Rub00] K. Rubin. Euler Systems. Annals of Mathematics Studies, 147. Hermann Weyl Lectures. The Institute for Advanced Study. Princeton University Press, Princeton, 2000.

[Sch82] P. Schneider. p-Adic Height Pairings I. Invent. Math., 69, 1982.

[Ser67] J.P. Serre. Local class field theory. In J. W. S. Cassels and A. Frohlich, editors, Algebraic Number Theory. Academic Press, 1967.

[Sil86] J. Silverman. The arithmetic of elliptic curves. Springer-Verlag New York, Inc., 1986.

[Sil94] J. Silverman. Advanced Topics in the arithmetic of elliptic curves. Springer-Verlag New York, Inc., 1994.

[Ven13] R. Venerucci. p-adic regulators and p-adic analytic families of modular forms. Ph.D. Thesis, University of Milan, 2013.

[Ven14] R. Venerucci. p-adic regulators and Hida p-adic L-functions. Preprint, 2014.

[Wil78] A. Wiles. Higher explicit reciprocity laws. Ann. Math. (2), 107(2), 1978.

R. Venerucci, Universität Duisburg-Essen, Fakultät für Mathematik, Mathematikcarrée, Thea-Leymann-Straße 9, 45127 Essen. E-mail address: rodolfo.venerucci@uni-due.de 